Vasile Ene, Quellenstrasse 18, 63571 Gelnhausen, Germany

e-mail: gabrielaene@hotmail.com

\title{
A STUDY OF SOME GENERAL INTEGRALS THAT CONTAINS THE WIDE DENJOY INTEGRAL
}

\begin{abstract}
In this paper, using Thomson's local systems, we introduce some very general integrals, each containing the wide Denjoy integral: the $\left[\mathcal{S}_{1} \mathcal{S}_{2} \mathcal{D}\right]$-integral (of Lusin type); the $\left[\mathcal{S}_{1} \mathcal{S}_{2} \mathcal{V}\right]$-integral (of variational type); the $\left[\mathcal{S}_{1} \mathcal{S}_{2} \mathcal{W}\right]$-integral (of Ward type); the $\left[\mathcal{S}_{1} \mathcal{S}_{2} \mathcal{R}\right]$-integral (of Riemann type); We prove that in certain conditions the integrals $\left[\mathcal{S}_{1} \mathcal{S}_{2} \mathcal{V}\right]$ and $\left[\mathcal{S}_{1} \mathcal{S}_{2} \mathcal{W}\right]$ are equivalent (it is shown that the first integral satisfies a Saks-Henstock type lemma). For the $\left[\mathcal{S}_{1} \mathcal{S}_{2} \mathcal{R}\right]$-integral we only show that it satisfies a quasi Saks Henstock type lemma (see Lemma 7.4). Finally, if $\mathcal{S}_{1}=\mathcal{S}_{o}^{+}$and $\mathcal{S}_{2}=\mathcal{S}_{o}^{-}$we obtain that the integrals $\left[\mathcal{S}_{o}^{+} \mathcal{S}_{o}^{-} \mathcal{V}\right]$, $\left[\mathcal{S}_{o}^{+} \mathcal{S}_{o}^{-} \mathcal{W}\right]$ and $\left[\mathcal{S}_{o}^{+} \mathcal{S}_{o}^{-} \mathcal{D}\right]$ are equivalent (in fact the $\left[\mathcal{S}_{o}^{+} \mathcal{S}_{o}^{-} \mathcal{D}\right]$-integral is exactly the wide Denjoy integral). But the equivalence of the three integrals with the $\left[\mathcal{S}_{o}^{+} \mathcal{S}_{o}^{-} \mathcal{R}\right]$-integral follows only if we assume the additional condition that the primitives of the $\left[\mathcal{S}_{o}^{+} \mathcal{S}_{o}^{-} \mathcal{R}\right]$-integral are continuous (see Theorem 11.1)
\end{abstract}

\section{Introduction}

It is well known that the Denjoy-Perron integral has a Riemann type definition. This was discovered independently by Henstock and Kurzweil, and it is called the Henstock-Kurzweil integral. Also the Denjoy-Perron integral allows characterizations of variational and of Ward type (these characterizations are due to Henstock). A very important fact in the theory of the Henstock-Kurzweil integral is the Saks-Henstock Lemma. Since 1968, Henstock suggested (see [5, p. 222]) that it is possible to obtain a Riemann type definition for the wide Denjoy integral. Starting from this suggestion and from the fact that an explicit theorem wasn't stated in Henstock's book, Lee and Soedijono introduced

Key Words: local systems, Henstock-Kurzweil integral, Ward integral, Denjoy integral

Mathematical Reviews subject classification: 26A39; 26A42; 26A45

Received by the editors January 8, 1999

*The author died on November 11, 1998; see Real. Anal. Exch. 241 (1989/99), 3. 
a Riemann type integral, called the $A H$-integral, about which they claimed that it was equivalent with the $\beta$-Ridder integral (note that the $\beta$-Ridder integral was also studied by Kubota, but he called it the $A D$ - integral). Indeed, by [12, Theorem 4.1] it follows that the $A D$ integral is contained in the $A H$ integral and the two integrals are equal. But that the converse is also true doesn't seem to follow from their Theorem 4.2. To prove this theorem they need to show the following facts:

1) $F$ is approximately continuous on $[a, b]$;

2) $F_{a p}^{\prime}(x)=f(x)$ a.e. on $[a, b]$;

3) $F \in V B G$ on $[a, b]$;

4) $F$ satisfies Lusin's condition $(N)$ on $[a, b]$;

5) $F \in[V B G]$ on $[a, b]$;

6) $[V B G] \cap(N)=[A C G]$ for approximately continuous functions on $[a, b]$,

where $F(x)=(A H) \int_{a}^{x} f(t) d t, x \in[a, b]$.

To show 1)-4) they use essentially a Saks-Henstock type lemma for the $A H$ integral, claiming that this lemma is easy to prove (I wasn't able to do so).

With or without a Saks-Henstock type lemma, I wasn't able to prove 1).

The items 2), 3) and 4) are true, but with different proofs (for 2) see Lemma 7.5; for 3) see Lemma 7.6; for 4) see Corollary 7.1).

In 4) there is also another error (it seems that the authors used the following statement, that is not true: if $\left\{\left[F\left(a_{i}\right), F\left(b_{i}\right)\right]\right\}_{i=1}^{n}$ is a finite set of nonoverlapping intervals then $\left\{\left[a_{i}, b_{i}\right]\right\}_{i=1}^{n}$ is also a set of nonoverlapping intervals).

The proof of 5 ) is not clear (because, if a function $F$ satisfies the "strong Lusin condition" then it isn't clear if $F$ is $V B$ on any subset $Z$ with $|Z|=0$; but it is true that $F$ is $V B G$ on $Z$, see Theorem 5.1 ; moreover, if a function is $V B$ on a set $A$ and on a set $B$, then it is not necessarily $V B$ on $A \cup B$ ).

Statement 6) is not true. Indeed, Sarkhel and Kar introduced the $(P A C)$ condition that is characterized as follows: A function $F$ is $(P A C)$ on a closed set $E$ if and only if $F \in[V B G] \cap(N)$ on $E$ (The generalized Banach-Zarecki theorem [19, Theorem 3.6]). In the same paper the authors constructed $a$ function $F:[a, b] \rightarrow \mathbb{R}$ with the following properties: $F$ is approximately continuous, $F \in(P A C)$, but $F \notin A C G$. It follows that the function $F$ from above is approximately continuous, $[V B G]$ and $(N)$ on $[a, b]$, but not $[A C G]$. Note however that $V B G \cap[C G] \cap(N)=[A C G]$ (and this follows indeed by the Banach-Zarecki theorem [15, p. 227]).

In this paper, using Thomson's local systems, we introduce some very general integrals, each containing the wide Denjoy integral: 
- the $\left[\mathcal{S}_{1} \mathcal{S}_{2} \mathcal{D}\right]$-integral (of Lusin type);

- the $\left[\mathcal{S}_{1} \mathcal{S}_{2} \mathcal{V}\right]$-integral (of variational type);

- the $\left[\mathcal{S}_{1} \mathcal{S}_{2} \mathcal{W}\right]$-integral (of Ward type);

- the $\left[\mathcal{S}_{1} \mathcal{S}_{2} \mathcal{R}\right]$-integral (of Riemann type);

We prove that in certain conditions, the integrals $\left[\mathcal{S}_{1} \mathcal{S}_{2} \mathcal{V}\right]$ and $\left[\mathcal{S}_{1} \mathcal{S}_{2} \mathcal{W}\right]$ are equivalent (it is shown that the first integral satisfies a Saks-Henstock type lemma). For the $\left[\mathcal{S}_{1} \mathcal{S}_{2} \mathcal{R}\right]$-integral we only show that it satisfies a quasi Saks Henstock type lemma (see Lemma 7.4).

Finally, if $\mathcal{S}_{1}=\mathcal{S}_{o}^{+}$and $\mathcal{S}_{2}=\mathcal{S}_{o}^{-}$we obtain that the integrals $\left[\mathcal{S}_{o}^{+} \mathcal{S}_{o}^{-} \mathcal{V}\right.$ ], $\left[\mathcal{S}_{o}^{+} \mathcal{S}_{o}^{-} \mathcal{W}\right]$ and $\left[\mathcal{S}_{o}^{+} \mathcal{S}_{o}^{-} \mathcal{D}\right]$ are equivalent (in fact the $\left[\mathcal{S}_{o}^{+} \mathcal{S}_{o}^{-} \mathcal{D}\right]$-integral is exactly the wide Denjoy integral). But the equivalence of the three integrals with the $\left[\mathcal{S}_{o}^{+} \mathcal{S}_{o}^{-} \mathcal{R}\right]$-integral follows only if we assume the additional condition, that the primitives of the $\left[\mathcal{S}_{o}^{+} \mathcal{S}_{o}^{-} \mathcal{R}\right]$-integral are continuous (see Theorem 11.1).

\section{Preliminaries}

We shall use the following well known classes of functions: $\mathcal{C}$ (continuous functions), $\mathcal{D}$ (Darboux functions), $\mathcal{B}_{1}$ (Baire one functions), $A C, V B,[A C G]$, $[A C G], A C G$ (the $A C G$ functions are not supposed to be continuous), $V B G$, $[C G]$ (or $\left.B_{1}^{*}\right)[V B G],(\mathrm{N})$ (Lusin's condition), $T_{2}, N^{-\infty}$ (see for example [15] or [1]). We denote by $\langle x, y\rangle$ the closed interval with the endpoints $x$ and $y$, where $x, y \in \mathbb{R}$.

Definition 2.1 (Thomson). [21, p. 3] A family $\mathcal{S}=\{\mathcal{S}(x)\}_{x \in \mathbb{R}}$ is said to be a local system if each $\mathcal{S}(x)$ is a collection of sets with the following properties:

(i) $\{x\} \notin \mathcal{S}(x)$;

(ii) If $\sigma_{x} \in \mathcal{S}(x)$ then $x \in \sigma_{x}$;

(iii) If $\sigma_{x} \in \mathcal{S}(x)$ and $\sigma_{x} \subset A$ then $A \in \mathcal{S}(x)$;

(iv) If $\sigma_{x} \in \mathcal{S}(x)$ and $\delta>0$ then $\sigma_{x} \cap(x-\delta, x+\delta) \in \mathcal{S}(x)$.

Definition 2.2. Let $\mathcal{S}_{1}=\left\{\mathcal{S}_{1}(x)\right\}_{x \in \mathbb{R}}$ and $\mathcal{S}_{2}=\left\{\mathcal{S}_{2}(x)\right\}_{x \in \mathbb{R}}$ be local systems and let $x \in \mathbb{R}, A \subset \mathbb{R}$.

- (Thomson, [21, p. 5]). We define the local system $\mathcal{S}_{1} \wedge \mathcal{S}_{2}=\left\{\left(\mathcal{S}_{1} \wedge\right.\right.$ $\left.\left.\mathcal{S}_{2}\right)(x)\right\}_{x \in \mathbb{R}}$ by $\left(\mathcal{S}_{1} \wedge \mathcal{S}_{2}\right)(x)=\mathcal{S}_{1}(x) \cap \mathcal{S}_{2}(x)$ (it is easy to verify that this is a local system). 
- (Thomson, [21, p. 37]). $\mathcal{S}_{1}$ is said to be bilateral at $x$ if $\sigma_{x}$ has $x$ as a bilateral accumulation point, whenever $\sigma_{x} \in \mathcal{S}_{1}(x)$. $\mathcal{S}_{1}$ is bilateral on $A$ if it is bilateral at each point of $A$.

- (Thomson, $\left[21\right.$, p. 18]). Let $\mathcal{S}_{\infty}=\left\{\mathcal{S}_{\infty}(x): x \in \mathbb{R}\right\}$ denote the local system defined at each point $x$ as $\mathcal{S}_{\infty}(x)=\{\sigma: \sigma$ contains $x$ and has $x$ as an accumulation point $\}$. We can define right and left versions of this, by writing: $\mathcal{S}_{\infty}^{+}(x)=\{\sigma: \sigma$ contains $x$ and has $x$ as a right accumulation point $\}$ and $\mathcal{S}_{\infty}^{-}(x)=\{\sigma: \sigma$ contains $x$ and has $x$ as a left accumulation point $\}$.

- Let $\mathcal{S}_{\infty, \infty}=\mathcal{S}_{\infty}^{+} \wedge \mathcal{S}_{\infty}^{-}$. Clearly $\mathcal{S}_{\infty, \infty}(x)=\{\sigma: \sigma$ contains $x$ and has $x$ as a bilateral accumulation point $\}$.

- $\mathcal{S}_{1}$ is said to be $\mathcal{S}_{2}$-filtering at $x$ if $\sigma_{x}^{\prime} \cap \sigma_{x}^{\prime \prime} \in \mathcal{S}_{2}(x)$ whenever $\sigma_{x}^{\prime}, \sigma_{x}^{\prime \prime} \in$ $\mathcal{S}_{1}(x) . \mathcal{S}_{1}$ is said to be $\mathcal{S}_{2}$-filtering on $A$ if it is so on each point of $A$.

- (Thomson, $[21$, p. 10]). $\mathcal{S}$ is said to be filtering at $x$ if it is $\mathcal{S}$-filtering at $x$.

- (Thomson, [21, p. 5]). We will write $\mathcal{S}_{1} \ll \mathcal{S}_{2}$ on $A$, if at every point $x \in A$ we have $\mathcal{S}_{1}(x) \subseteq \mathcal{S}_{2}(x)$.

- If $\mathcal{S}_{1} \ll \mathcal{S}_{\infty}^{+}$and $\mathcal{S}_{2} \ll \mathcal{S}_{\infty}^{-}$then we define the following local system: $\left(\mathcal{S}_{1} ; \mathcal{S}_{2}\right)=\left\{\left(\mathcal{S}_{1} ; \mathcal{S}_{2}\right)(x)\right\}_{x \in \mathbb{R}}$, where $\left(\mathcal{S}_{1} ; \mathcal{S}_{2}\right)(x)=\{S: x \in S$ and there exist $\delta>0, A \in \mathcal{S}_{1}(x)$ and $B \in \mathcal{S}_{2}(x)$ such that $((x-\delta, x) \cap B) \cup((x, x+$ $\delta) \cap A) \subset S\}$.

Remark 2.1. If $\mathcal{S}$ is $\mathcal{S}_{\infty, \infty}$-filtering then it is a bilateral local system.

Definition 2.3. Let $\mathcal{S}=\{\mathcal{S}(x)\}_{x \in \mathbb{R}}$ be a local system. Let $F:[a, b] \rightarrow \mathbb{R}$ and $t \in[a, b]$.

- $F$ is said to be $\mathcal{S C}(\mathcal{S}$-continuous) at $t$ if for every $\epsilon>0$ there exists $\sigma_{t} \in \mathcal{S}(t)$ such that $|F(x)-F(t)|<\epsilon$, whenever $x \in \sigma_{t} \cap[a, b] . F$ is said to be $\mathcal{S C}$ on a set $A \subset[a, b]$ if it is so at each point $t \in A$.

- $F$ is said to be $\mathcal{S}$-upper (respectively lower) semi-continuous at $t$ if for every $\epsilon>0$ there exists $\sigma_{t} \in \mathcal{S}(t)$ such that $F(t)-F(x)<\epsilon$ (respectively $F(t)-F(x)>-\epsilon)$, whenever $x \in \sigma_{t} \cap[a, b] . F$ is said to be $\mathcal{S}$-upper (respectively lower) semi-continuous on a set $A \subset[a, b]$ if it is so at each point $t \in A$.

Remark 2.2. With the above notations we have: 
(i) If $F$ is $\mathcal{S C}$ at $t \in[a, b]$ then $F$ is both, $\mathcal{S}$-upper semi-continuous and $\mathcal{S}$-lower semi-continuous at $t$. If $\mathcal{S}$ is a filtering local system the converse is also true.

(ii) Definition 2.3 is a slight modification of Thomson's definitions (31.1) and (31.3) of [21, pp. 70-71].

Definition 2.4. Let $\mathcal{S}=\{\mathcal{S}(x)\}_{x \in \mathbb{R}}$ be a bilateral local system, and let $F$ : $[a, b] \rightarrow \mathbb{R}$.

- $F$ is said to be right (respectively left) $\mathcal{S C}$ at a point $x \in[a, b)$ (respectively $x \in(a, b])$, if for every $\epsilon>0$ there exists $\sigma_{x} \in \mathcal{S}(x)$ such that $|F(t)-F(x)|<\epsilon$, whenever $t \in \sigma_{x} \cap[x, b)$ (respectively $t \in \sigma_{x} \cap(a, x]$ ). $F$ is said to be right (respectively left) $\mathcal{S C}$ on a set $A \subset[a, b)$ (respectively $A \subset(a, b])$, if it is so at each point $x \in A$. If $F$ is right $\mathcal{S C}$ on $[a, b)$ and left $\mathcal{S C}$ on $(a, b]$, we say that $F$ is bilateral $\mathcal{S C}$ on $[a, b]$.

- $F$ is said to be right (respectively left) $\mathcal{S}$ - upper semi-continuous at a point $x \in[a, b)$ (respectively $x \in(a, b]$ ), if for every $\epsilon>0$ there exists $\sigma_{x} \in \mathcal{S}(x)$ such that $F(t)-F(x)<\epsilon$, whenever $t \in \sigma_{x} \cap[x, b)$ (respectively $\left.t \in \sigma_{x} \cap(a, x]\right)$. $F$ is said to be right (respectively left) $\mathcal{S}$-upper semi-continuous on a set $A \subset[a, b)$ (respectively $A \subset(a, b])$, if it is so at each point $x \in A$.

- $F$ is said to be right (respectively left) $\mathcal{S}$ - lower semi-continuous at a point $x \in[a, b)$ (respectively $x \in(a, b]$ ), if for every $\epsilon>0$ there exists $\sigma_{x} \in \mathcal{S}(x)$ such that $F(t)-F(x)>-\epsilon$, whenever $t \in \sigma_{x} \cap[x, b)$ (respectively $\left.t \in \sigma_{x} \cap(a, x]\right) . \quad F$ is said to be right (respectively left) $\mathcal{S}$-lower semi-continuous on a set $A \subset[a, b)$ (respectively $A \subset(a, b]$ ), if it is so at each point $x \in A$.

- $F$ is said to be $\mathcal{S C}_{i}$ at $t \in[a, b]$ if for every $\epsilon>0$ there exists $\sigma_{t} \in \mathcal{S}(t)$ such that $F(x) \leq F(t)+\epsilon$ for $x \in \sigma_{t} \cap[a, t]$, and $F(t)-\epsilon \leq F(y)$ for $y \in \sigma_{t} \cap[t, b] . F$ is said to be $\mathcal{S C}_{d}$ at $t$ if $-F$ is $\mathcal{S C}_{i}$ at $t . F$ is said to be $\mathcal{S C}_{i}$ (respectively $\mathcal{S C}_{d}$ ) on a set $A$ if it is so at each point of $A$.

Lemma 2.1. Let $\mathcal{S}_{1}=\left\{\mathcal{S}_{1}(x)\right\}_{x \in \mathbb{R}}$ and $\mathcal{S}_{2}=\left\{\mathcal{S}_{2}(x)\right\}_{x \in \mathbb{R}}$ be local systems such that $\mathcal{S}_{1} \ll \mathcal{S}_{\infty}^{+}$on $[a, b)$ and $\mathcal{S}_{2} \ll \mathcal{S}_{\infty}^{-}$on $(a, b]$. Let $F:[a, b] \rightarrow \mathbb{R}$ and $x \in[a, b]$.

(i) The following assertions are equivalent:

a) $F$ is $\left(\mathcal{S}_{1} ; \mathcal{S}_{2}\right) \mathcal{C}$ at $x$;

b) $F$ is left $\mathcal{S}_{2} \mathcal{C}$ at $x$ if $x \in(a, b]$, and $F$ is right $\mathcal{S}_{1} \mathcal{C}$ at $x$ if $x \in[a, b)$. 
(ii) The following assertions are equivalent:

a) $F$ is $\left(\mathcal{S}_{1} ; \mathcal{S}_{2}\right) \mathcal{C}_{i}$ at $x$;

b) $F$ is left $\mathcal{S}_{2}$-upper semi-continuous at $x$ if $x \in(a, b]$, and $F$ is right $\mathcal{S}_{1}$-lower semi-continuous at $x$ if $x \in[a, b)$.

Proof. Evident.

Remark 2.3. Let $F:[a, b] \rightarrow \mathbb{R}$.

(i) $F$ is right $\mathcal{S}_{\infty}^{+}$lower semicontinuous at a point $x \in[a, b)$ if and only if $F$ is $\mathcal{S}_{\infty}^{+}$lower semicontinuous at $x$.

(ii) $F$ is left $\mathcal{S}_{\infty}^{-}$lower semicontinuous at a point $x \in[a, b)$ if and only if $F$ is $\mathcal{S}_{\infty}^{-}$lower semicontinuous at $x$.

(iii) $F$ is lower internal (this condition is due to Garg, see [1, p. 33]) if and only if $F$ is $\left(\mathcal{S}_{\infty}^{+} ; \mathcal{S}_{\infty}^{-}\right) \mathcal{C}_{i}$ on $[a, b]$. (see (i), (ii) and Lemma 2.1, (ii)).

Lemma 2.2. Let $\mathcal{S}_{1}=\left\{\mathcal{S}_{1}(x)\right\}_{x \in \mathbb{R}}$ and $\mathcal{S}_{2}=\left\{\mathcal{S}_{2}(x)\right\}_{x \in \mathbb{R}}$ be local systems such that $\mathcal{S}_{1} \ll \mathcal{S}_{\infty}^{+}$on $[a, b)$ and $\mathcal{S}_{2} \ll \mathcal{S}_{\infty}^{-}$on $(a, b]$. Let $F, G:[a, b] \rightarrow \mathbb{R}$ and $c \in(a, b)$. Let $F_{1}:[a, c] \rightarrow \mathbb{R}, F_{1}(x)=F(x)$, and let $F_{2}:[c, b] \rightarrow \mathbb{R}$, $F_{2}(x)=F(x)$.

(i) If $\mathcal{S}_{1}=\mathcal{S}_{2}$ and $F$ is $\mathcal{S}_{1} \mathcal{C}$ at $x \in[a, b]$ then $F$ is $\left(\mathcal{S}_{1} ; \mathcal{S}_{2}\right) \mathcal{C}$ at $x$;

(ii) Suppose that $\mathcal{S}_{1}=\mathcal{S}_{2}$ is filtering on $[a, b]$. Then $\mathcal{S}_{1} \mathcal{C}=\left(\mathcal{S}_{1} ; \mathcal{S}_{2}\right) \mathcal{C}$ on $[a, b]$.

(iii) $F_{1} \in\left(\mathcal{S}_{1} ; \mathcal{S}_{2}\right) \mathcal{C}$ (resp. $\left.\left(\mathcal{S}_{1} ; \mathcal{S}_{2}\right) \mathcal{C}_{i}\right)$ on $[a, c]$ and $F_{2} \in\left(\mathcal{S}_{1} ; \mathcal{S}_{2}\right) \mathcal{C}$ (resp. $\left.\left(\mathcal{S}_{1} ; \mathcal{S}_{2}\right) \mathcal{C}_{i}\right)$ on $[c, b]$ if and only if $F$ is $\left(\mathcal{S}_{1} ; \mathcal{S}_{2}\right) \mathcal{C}\left(\right.$ resp. $\left.\left(\mathcal{S}_{1} ; \mathcal{S}_{2}\right) \mathcal{C}_{i}\right)$ on $[a, b]$.

(iv) $\left(\mathcal{S}_{1} ; \mathcal{S}_{2}\right) \mathcal{C} \subset\left(\mathcal{S}_{1} ; \mathcal{S}_{2}\right) \mathcal{C}_{i} \cap\left(\mathcal{S}_{1} ; \mathcal{S}_{2}\right) \mathcal{C}_{d}$ on $[a, b]$

(v) $\mathcal{B}_{1} \cap\left(\mathcal{S}_{1} ; \mathcal{S}_{2}\right) \mathcal{C}_{i} \subset \mathcal{D}_{-} \mathcal{B}_{1}$ on $[a, b]$.

(vi) $\mathcal{B}_{1} \cap\left(\mathcal{S}_{1} ; \mathcal{S}_{2}\right) \mathcal{C} \subset \mathcal{B}_{1} \cap\left(\mathcal{S}_{1} ; \mathcal{S}_{2}\right) \mathcal{C}_{i} \cap\left(\mathcal{S}_{1} ; \mathcal{S}_{2}\right) \mathcal{C}_{d} \subset \mathcal{D B}_{1}$ on $[a, b]$.

(vii) Suppose that $\mathcal{S}_{1}$ is $\mathcal{S}_{\infty}^{+}$-filtering on $[a, b)$ and $\mathcal{S}_{2}$ is $\mathcal{S}_{\infty}^{-}$-filtering on $(a, b]$. Then on $[a, b]$ we have

- $\left(\mathcal{S}_{1} ; \mathcal{S}_{2}\right) \mathcal{C}_{i}+\left(\mathcal{S}_{1} ; \mathcal{S}_{2}\right) \mathcal{C}_{i} \subset\left(\mathcal{S}_{\infty}^{+} ; \mathcal{S}_{\infty}^{-}\right) \mathcal{C}_{i}$

- $\left(\mathcal{B}_{1} \cap\left(\mathcal{S}_{1} ; \mathcal{S}_{2}\right) \mathcal{C}_{i}\right)+\left(\mathcal{B}_{1} \cap\left(\mathcal{S}_{1} ; \mathcal{S}_{2}\right) \mathcal{C}_{i}\right) \subseteq \mathcal{B}_{1} \cap\left(\mathcal{S}_{\infty}^{+} ; \mathcal{S}_{\infty}^{-}\right) \mathcal{C}_{i}=\mathcal{D}_{-} \mathcal{B}_{1} ;$

- $\left(\mathcal{S}_{1} ; \mathcal{S}_{2}\right) \mathcal{C}+\left(\mathcal{S}_{1} ; \mathcal{S}_{2}\right) \mathcal{C} \subset\left(\mathcal{S}_{\infty}^{+} ; \mathcal{S}_{\infty}^{-}\right) \mathcal{C}$; 


$$
\text { - }\left(\mathcal{B}_{1} \cap\left(\mathcal{S}_{1} ; \mathcal{S}_{2}\right) \mathcal{C}\right)+\left(\mathcal{B}_{1} \cap\left(\mathcal{S}_{1} ; \mathcal{S}_{2}\right) \mathcal{C}\right) \subseteq \mathcal{B}_{1} \cap\left(\mathcal{S}_{\infty}^{+} ; \mathcal{S}_{\infty}^{-}\right) \mathcal{C}=\mathcal{D} \mathcal{B}_{1}
$$

Proof. See Theorem 2.5.1, (i), (iv) of [1] and Remark 2.3, (iii).

Lemma 2.3. Let $F:[a, b] \rightarrow \mathbb{R}$. If $F \in[\underline{A C G}] \cap\left(\mathcal{S}_{\infty}^{+} ; \mathcal{S}_{\infty}^{-}\right) \mathcal{C}_{i}$ on $[a, b]$ and $F_{a p}^{\prime}(x) \geq 0$ a.e. on $[a, b]$ then $F$ is increasing on $[a, b]$.

Proof. We have $[\underline{A C G}] \subset[V B G] \subset T_{2}$ (see Theorem 2.11.1, (vi) and Theorem 2.18.9 of [1]) and $[\underline{A C G}] \subset N^{-\infty}$ (see for example Theorem 2.20.1 and Lemma 2.21.1 of [1]). Let $Q$ be a perfect subset of $[a, b]$. Since $F \in[V B G]$, there exists a portion $(\alpha, \beta) \cap Q \neq \emptyset$ of $Q$ such that $F \in V B$ on it (see Theorem 1.9.1, (ii) of [1]). Then $F_{/ Q}$ is continuous nearly everywhere on $(\alpha, \beta) \cap Q$, hence $F \in \mathcal{B}_{1}$ on $[a, b]$ (see Theorem 2.2.1 of [1]). By Lemma 2.2, $F \in \mathcal{D}_{-} \mathcal{B}_{1} T_{2} \cap N^{-\infty}$ on $[a, b]$. Now Corollary 4.3.1. of [1] completes our proof.

Corollary 2.1. Let $F:[a, b] \rightarrow \mathbb{R}$. If $F \in[A C G] \cap\left(\mathcal{S}_{\infty}^{+} ; \mathcal{S}_{\infty}^{-}\right) \mathcal{C}$ on $[a, b]$ and $F_{a p}^{\prime}(x)=0$ a.e. on $[a, b]$ then $F$ is constant on $[a, b]$.

\section{Examples of Local Systems}

We recall the following local systems.

- $\mathcal{S}_{o}^{+}=\left\{\mathcal{S}_{o}^{+}(x)\right\}_{x \in \mathbb{R}}$, where $S_{o}^{+}(x)=\{U: U$ is a right neighborhood of $x\}$.

- $\mathcal{S}_{o}^{-}=\left\{\mathcal{S}_{o}^{-}(x)\right\}_{x \in \mathbb{R}}$, where $S_{o}^{-}(x)=\{U: U$ is a left neighborhood of $x\}$.

- $\mathcal{S}_{a p}^{+}=\left\{\mathcal{S}_{a p}^{+}(x)\right\}_{x \in \mathbb{R}}$, where $S_{a p}^{+}(x)=\left\{S: x \in S\right.$ and $\left.\underline{d}_{+}^{i}(S ; x)=1\right\}$.

- $\mathcal{S}_{a p}^{-}=\left\{\mathcal{S}_{a p}^{-}(x)\right\}_{x \in \mathbb{R}}$, where $S_{a p}^{-}(x)=\left\{S: x \in S\right.$ and $\left.\underline{d}_{-}^{i}(S ; x)=1\right\}$.

- For $\alpha \in(0,1)$ let $\mathcal{S}_{\alpha}^{+}=\left\{\mathcal{S}_{\alpha}^{+}(x)\right\}_{x \in \mathbb{R}}$, where $S_{\alpha}^{+}(x)=\{S: x \in S$ and $\left.\underline{d}_{+}^{i}(S ; x) \geq \alpha\right\}$.

- For $\alpha \in(0,1)$ let $\mathcal{S}_{\alpha}^{-}=\left\{\mathcal{S}_{\alpha}^{-}(x)\right\}_{x \in \mathbb{R}}$, where $S_{\alpha}^{-}(x)=\{S: x \in S$ and $\left.\underline{d}_{-}^{i}(S ; x) \geq \alpha\right\}$.

- $\mathcal{S}_{\text {pro,o }}^{+}=\left\{\mathcal{S}_{\text {pro,o }}^{+}(x)\right\}_{x \in \mathbb{R}}$, where $\mathcal{S}_{\text {pro,o }}^{+}(x)=\{A: x \in A$ and there is a measurable set $E \subseteq A$ such that $d^{+}(E, x)=1$ and $\left.d_{+}(E, x)>0\right\}$;

- $\mathcal{S}_{\text {pro,o }}^{-}=\left\{\mathcal{S}_{\text {pro }, o}^{-}(x)\right\}_{x \in \mathbb{R}}$, where $\mathcal{S}_{\text {pro,o }}^{-}(x)=\{A: x \in A$ and there is a measurable set $E \subseteq A$ such that $d^{-}(E, x)=1$ and $\left.d_{-}(E, x)>0\right\}$; 
- $\mathcal{S}_{\text {pro }}^{+}=\left\{\mathcal{S}_{\text {pro }}^{+}(x)\right\}_{x \in \mathbb{R}}$, where $\mathcal{S}_{\text {pro,o }}^{+}(x)=\{A: x \in A$ and there is a measurable set $E \subset A$ such that $E \cap P \in \mathcal{S}_{\text {pro,o }}^{+}(x)$ whenever $P$ is a measurable set in $\mathcal{S}_{\text {pro,o }}^{+}(x)$;

- $\mathcal{S}_{\text {pro }}^{-}=\left\{\mathcal{S}_{\text {pro }}^{-}(x)\right\}_{x \in \mathbb{R}}$, where $\mathcal{S}_{\text {pro,o }}^{-}(x)=\{A: x \in A$ and there is a measurable set $E \subset A$ such that $E \cap P \in \mathcal{S}_{\text {pro,o }}^{-}(x)$ whenever $P$ is a measurable set in $\mathcal{S}_{\text {pro,o }}^{-}(x)$;

(Here $\underline{d}_{+}^{i}$ and $\underline{d}_{-}^{i}$ are the interior right respectively left densities of the set $S$ at $x$.)

Remark 3.1. The local systems $\mathcal{S}_{o}^{+}, \mathcal{S}_{o}^{-}, \mathcal{S}_{a p}^{+}, \mathcal{S}_{a p}^{-}, \mathcal{S}_{\alpha}^{+}$and $\mathcal{S}_{\alpha}^{-}$were defined by Thomson in [21, pp. 18, 22]. The local systems $\mathcal{S}_{\text {pro,o }}^{+}, \mathcal{S}_{\text {pro oo }}^{-}, \mathcal{S}_{\text {pro }}^{+}$and $\mathcal{S}_{\text {pro }}^{-}$were used by Filipczak in [3] (p. 172; with different names), who gives credit for their introduction to Sarkhel and De. In fact in Sarkhel and De's terminology [18, pp. 30-32], a set $A \in \mathcal{S}_{\text {pro }}^{+}(x)$ if and only if $x \in A$ and $\mathbb{R} \backslash A$ is sparse at $x$ on the right.

Remark 3.2. With the above notations we have:

(i) The local systems $\mathcal{S}_{o}^{+}, \mathcal{S}_{o}^{-}, \mathcal{S}_{a p}^{+}$and $\mathcal{S}_{a p}^{-}$are filtering.

(ii) If $\alpha>\frac{1}{2}$ then $\mathcal{S}_{\alpha}^{+}$is $\mathcal{S}_{\infty}^{+}$-filtering, and $\mathcal{S}_{\alpha}^{-}$is $\mathcal{S}_{\infty}^{-}$-filtering.

(iii) $\left(\mathcal{S}_{o}^{+} ; \mathcal{S}_{o}^{-}\right) \mathcal{C}=\mathcal{C}$;

(iv) $\left(\mathcal{S}_{a p}^{+} ; \mathcal{S}_{a p}^{-}\right) \mathcal{C}=\mathcal{C}_{a p}$, where $\mathcal{C}_{a p}$ denotes the class of approximately continuous functions.

(v) $\mathcal{S}_{\text {pro }}^{+}$and $\mathcal{S}_{\text {pro }}^{-}$are filtering. (Indeed. Let $P$ be a measurable set in $\mathcal{S}_{\text {pro oo }}^{+}(x)$, and let $A_{1}, A_{2} \in \mathcal{S}_{\text {pro }}^{+}(x), A=A_{1} \cap A_{2}$. Then $x \in A$ and there exists $E_{i}$ measurable, $E_{i} \subset A_{i}$ such that $E_{i} \cap P \in \mathcal{S}_{\text {pro,o }}^{+}(x), i=1,2$. Let $E=E_{1} \cap E_{2}$. Then $E \cap P=E_{1} \cap\left(E_{2} \cap P\right) \in \mathcal{S}_{\text {pro }, o}^{+}(x)$. Hence $A \in \mathcal{S}_{\text {pro }}^{+}(x)$.)

(vi) $\left(\mathcal{S}_{\text {pro }}^{+} ; \mathcal{S}_{\text {pro }}^{-}\right) \mathcal{C}=\mathcal{C}_{\text {pro }}$, where $\mathcal{C}_{\text {pro }}$ is the proximal continuity introduced by Sarkhel and De in [18].

\section{A Fundamental Lemma}

Lemma 4.1. Let $P$ be a perfect nowhere dense subset of $[a, b], a, b \in P$, and let $\delta: P \rightarrow(0,+\infty)$. Then there exists a finite set $\mathcal{A}=\{([y, z] ; x): x \in$ $\{y, z\} \subset P, x$ is a limit point of $[y, z] \cap P$ and $[y, z] \subset(x-\delta(x), x+\delta(x))\}$, such that $\cup_{([y, z], x) \in \mathcal{A}}[y, z] \supseteq P$. 
Proof. Let $\left\{\left(a_{i}, b_{i}\right)\right\}, i=\overline{1, \infty}$ be the intervals contiguous to $P$, and let $\eta:[a, b] \rightarrow(0,+\infty)$,

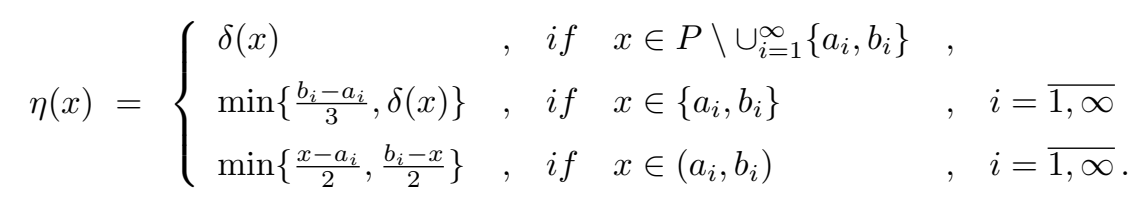

Let $\pi$ be a $\eta$-fine partition of $[a, b]$ (i.e., $a=x_{o}<x_{1}<\ldots<x_{n}=b$ and $t_{i} \in\left[x_{i-1}, x_{i}\right] \subset\left(t_{i}-\eta\left(t_{i}\right), t_{i}+\eta_{i}\left(t_{i}\right)\right)$; that such a partition exists follows for example by [1, p. 87]. Let $\pi_{1}=\{(I, x) \in \pi: x \in P\}$ and $\pi_{2}=\{(I, x) \in \pi$ : $x \notin P\}$. Clearly $\pi=\pi_{1} \cup \pi_{2}$. If $(I, x) \in \pi_{2}$ then $x \notin P$. Then there exists some $i$ such that $x \in\left(a_{i}, b_{i}\right)$, hence $x \in I \subset(x-\eta(x), x+\eta(x)) \subset\left(a_{i}, b_{i}\right)$. It follows that

$$
\cup_{(I, x) \in \pi_{1}} I \supset P .
$$

Let $(I, x) \in \pi_{1}$ and let $[y, z]$ be the smallest closed interval that contains $I \cap P$. We have three situations:

1) Suppose that $x \in P \backslash \cup_{i=1}^{\infty}\left\{a_{i}, b_{i}\right\}$. Then $x, y, z \in P$ and $y \leq x \leq z$. If $x \in\{y, z\}$ then $x$ is a limit point of $[y, z] \cap P$, and if $x \in(y, z)$ then $[y, x] \cap P$ and $[x, z] \cap P$ have $x$ as a limit point.

2) Suppose that $x=a_{i}$ for some $i$. Then $z=a_{i}$ and $\left[y, a_{i}\right] \cap P$ has $a_{i}$ as a limit point.

3) Suppose that $x=b_{i}$ for some $i$. Then $y=b_{i}$ and $\left[b_{i}, z\right] \cap P$ has $b_{i}$ as a limit point.

By 1), 2), 3) and (1) it follows that there exists a finite set $\mathcal{A}$ with the required properties.

Definition 4.1. Let $Z$ be a real set.

- Let $\mathcal{P}_{Z}$ be the collection of all sequences $\left\{Z_{n}\right\}_{n}$ of sets whose union is $Z$. If in addition each $Z_{n}$ is closed then we denote this collection by $\overline{\mathcal{P}}_{Z}$.

- Let $\left\{Z_{n}\right\}_{n} \in \mathcal{P}_{Z}$ and $\delta_{n}: Z_{n} \rightarrow(0,+\infty)$. Let $\beta=\beta\left(\left\{Z_{n}\right\},\left\{\delta_{n}\right\}\right)$ denote the collection of all tag intervals $([x, y], t), t \in\{x, y\} \subset Z$, such that $x, y \in Z_{n}$ and $y-x<\delta_{n}(t)$ whenever $t \in Z_{n}$. Let's denote the collection of all $\beta$ by $\mathcal{B}_{Z}$. If the collection $\mathcal{P}_{Z}$ is replaced by $\overline{\mathcal{P}}_{Z}$ then we denote this collection by $\overline{\mathcal{B}}_{Z}$. (The collection $\overline{\mathcal{B}}_{\mathbb{R}}$ was defined by Thomson in [20, p. 115], but he called it $C$ ).

- Let $\mathcal{B}_{o}$ denote the collection of all $\mathcal{B}_{Z}$, with $|Z|=0$. 
- Let $\beta \in \mathcal{B}_{Z}$ for some real set $Z$, and let $\pi=\left\{\left(I_{k}, t_{k}\right)\right\}_{k=1}^{m}$ be a finite subset of $\beta$. $\pi$ is said to be a $\beta$-partial partition of $Z$ if the intervals $\left\{I_{k}\right\}_{k}$ are nonoverlapping.

Definition 4.2. Let $P$ be a real set. We denote by

- $I s^{+}(P)=\{x \in P: x$ is a right isolated point of $P\}$;

- $I s^{-}(P)=\{x \in P: x$ is a left isolated point of $P\}$;

- $I s(P)=I s^{+}(P) \cup I s^{-}(P)$.

Let $\left\{P_{n}\right\}_{n}$ be a sequence of real sets. We denote by

- $I s^{+}\left(\left\{P_{n}\right\}\right)=\cup_{n=1}^{\infty} I s^{+}\left(P_{n}\right)$;

- $I s^{-}\left(\left\{P_{n}\right\}\right)=\cup_{n=1}^{\infty} I s^{-}\left(P_{n}\right)$;

- $I s\left(\left\{P_{n}\right\}\right)=\cup_{n=1}^{\infty} I s\left(P_{n}\right)$ (this set is countable, see [15, p. 260]).

Definition 4.3. Let $\left\{P_{n}\right\}_{n} \in \overline{\mathcal{P}}_{[a, b]}$ and let $\delta_{n}: P_{n} \rightarrow(0,+\infty)$. Let $\mathcal{S}_{1}=$ $\left\{\mathcal{S}_{1}(x)\right\}_{x \in \mathbb{R}}$ be a local system such that $\mathcal{S}_{1} \ll \mathcal{S}_{\infty}^{+}$on $[a, b]$, and let $\mathcal{S}_{2}=$ $\left\{\mathcal{S}_{2}(x)\right\}_{x \in \mathbb{R}}$ be a local system such that $\mathcal{S}_{2} \ll \mathcal{S}_{\infty}^{-}$on $[a, b]$. For each $x \in$ $I s^{+}\left(P_{n} \cap[a, b)\right)$ let $\sigma_{x, n}^{(1)} \in \mathcal{S}_{1}(x)$, and for each $x \in I s^{-}\left(P_{n} \cap(a, b]\right)$ let $\sigma_{x, n}^{(2)} \in$ $\mathcal{S}_{2}(x)$.

(i) Let $\alpha=\alpha\left(\left\{P_{n}\right\},\left\{\delta_{n}\right\}, \sigma_{x, n}^{(1)}, \sigma_{x, n}^{(2)}\right)$ denote the collection of all tag intervals $([x, y], t), t \in\{x, y\} \subset[a, b]$ such that:

- For $t=x \in P_{n}$

* $y \in\left(t, t+\delta_{n}(t)\right) \cap P_{n}$ whenever $t$ is a right accumulation point for $P_{n}$;

* $y \in \sigma_{t, n}^{(1)}$ whenever $t \in I s^{+}\left(P_{n} \cap[a, b)\right)$;

- For $t=y \in P_{n}$

$* x \in\left(t-\delta_{n}(t), t\right) \cap P_{n}$ whenever $t$ is a left accumulation point for $P_{n}$;

$* x \in \sigma_{t, n}^{(2)}$ whenever $t \in I s^{-}\left(P_{n} \cap(a, b]\right)$.

(ii) We denote the collection of all $\alpha$ by $\mathcal{A}\left(\overline{\mathcal{P}}_{[a, b]} ; \mathcal{S}_{1} ; \mathcal{S}_{2}\right)$

(iii) Let $A$ be a real set such that $A \supset I s\left(\left\{P_{n}\right\}\right)$. Let $\sigma_{t}^{(1)} \in \mathcal{S}_{1}(t)$, with $t \in$ $A \cap[a, b)$, and let $\sigma_{t}^{(2)} \in \mathcal{S}_{2}(t)$, with $t \in A \cap(a, b]$. Let $\beta_{A}=\beta_{A}\left(\sigma_{t}^{(1)}, \sigma_{t}^{(2)}\right)$ denote the collection of all tag intervals $([x, y], t), t \in\{x, y\} \subset[a, b]$ such that: 
- $x=t$ and $y \in \sigma_{t}^{(1)}$ whenever $t \in A \cap[a, b)$;

- $y=t$ and $x \in \sigma_{t}^{(2)}$ whenever $t \in A \cap(a, b]$.

(iv) We denote the collection of all $\beta_{A}$ by $\mathcal{B}_{A}\left(\mathcal{S}_{1} ; \mathcal{S}_{2}\right)$.

(v) We denote by $\mathcal{B}\left(\overline{\mathcal{P}}_{[a, b]} ; \mathcal{S}_{1}, \mathcal{S}_{2}\right)=\overline{\mathcal{B}}_{[a, b]} \cup\left(\cup\left\{\mathcal{B}_{A}\left(\mathcal{S}_{1}, \mathcal{S}_{2}\right): A\right.\right.$ is a countable subset of $[a, b]$ that contains $\left.\left.I s\left(\left\{P_{n}\right\}\right)\right\}\right)$.

(vi) Let $\beta \in \mathcal{B}\left(\overline{\mathcal{P}}_{[a, b]} ; \mathcal{S}_{1} ; \mathcal{S}_{2}\right)$ and let $\pi=\left\{\left(I_{k}, t_{k}\right)\right\}_{k=1}^{m}$ be a finite subset of $\beta . \pi$ is said to be a $\beta$-partial partition of $[a, b]$ if the intervals $\left\{I_{k}\right\}_{k}$ are nonoverlapping. If in addition $\cup_{k=1}^{m} I_{k}=[a, b]$ then $\pi$ is said to be a $\beta$-partition of $[a, b]$.

Remark 4.1. With the notations of Definition 4.3 we have:

(i) $\mathcal{B}\left(\overline{\mathcal{P}}_{[a, b]} ; \mathcal{S}_{1}, \mathcal{S}_{2}\right) \supset \mathcal{A}\left(\overline{\mathcal{P}}_{[a, b]} ; \mathcal{S}_{1}, \mathcal{S}_{2}\right)$.

(ii) $\overline{\mathcal{B}}_{[a, b]} \cup \mathcal{B}_{I s\left(\left\{P_{n}\right\}\right)}\left(\mathcal{S}_{o}^{+} ; \mathcal{S}_{o}^{-}\right)$is the family $P C$, introduced by Henstock [21, p. 115].

(iii) $\mathcal{A}\left(\overline{\mathcal{P}}_{[a, b]} ; \mathcal{S}_{a p}^{+} ; \mathcal{S}_{a p}^{-}\right)$was defined in $[12]$

Lemma 4.2 (Fundamental lemma). For each $\beta \in \mathcal{A}\left(\overline{\mathcal{P}}_{[a, b]} ; \mathcal{S}_{1} ; \mathcal{S}_{2}\right)$, there exists a $\beta$-partition of $[a, b]$ (see Definition 4.3). Particularly, the assertion is true for every $\beta \in \mathcal{B}\left(\overline{\mathcal{P}}_{[a, b]} ; \mathcal{S}_{1} ; \mathcal{S}_{2}\right)$

Proof. We shall use the Romanovski Lemma (see for example [1, p. 10]). Let $\mathcal{A}=\left\{(p, q) \subseteq(a, b):\left[p_{1}, q_{1}\right]\right.$ admits a $\beta$-partition whenever $\left.\left(p_{1}, q_{1}\right) \subseteq(p, q)\right\}$.

(i) If $(p, q) \in \mathcal{A}$ and $(q, r) \in \mathcal{A}$ then clearly $(p, r) \in \mathcal{A}$. of $\mathcal{A}$.

(ii) If $(p, q) \in \mathcal{A}$ and $\left(p_{1}, q_{1}\right) \subset(p, q)$ then $\left(p_{1}, q_{1}\right) \in \mathcal{A}$ (see the definition

(iii) Let $(c, d) \subseteq(a, b)$ such that $(p, q) \in \mathcal{A}$ whenever $[p, q] \subset(c, d)$. We show that $(c, d) \in \mathcal{A}$. Let $c \in P_{n}$. Let $c_{1} \in\left(c, c+\delta_{n}(c)\right) \cap P_{n} \cap(c,(c+d) / 2)$ if $c$ is a right accumulation point for $P_{n}$, and let $c_{1} \in \sigma_{c}^{(1)} \cap(c,(c+d) / 2)$ if $c$ is right isolated in $[a, b) \cap P_{n}$. Then $\left(\left[c, c_{1}\right], c\right) \in \beta$. Similarly we find $d_{1} \in((c+d) / 2, d)$ such that $\left(\left[d_{1}, d\right], d\right) \in \beta$. But $\left(c_{1}, d_{1}\right) \in \mathcal{A}$ and $[c, d]=$ $\left[c, c_{1}\right] \cup\left[c_{1}, d_{1}\right] \cup\left[d_{1}, d\right]$. Therefore $[c, d]$ admits a $\beta$-partition. Analogously we obtain that $\left[c_{2}, d_{2}\right]$ admits a $\beta$-partition, whenever $\left(c_{2}, d_{2}\right) \subset(c, d)$. Hence $(c, d) \in \mathcal{A}$.

(iv) Let $E \subset[a, b]$ be a perfect set such that all intervals contiguous to $E$ are contained in $\mathcal{A}$. We show that there exists $(p, q) \in \mathcal{A}$ such that $E \cap(p, q) \neq \emptyset$. Since $E=\cup_{n=1}^{\infty}\left(E \cap P_{n}\right)$, by the Baire Category Theorem (see for example [1, p. 10]) it follows that there exists a positive integer $n$ and an interval $(p, q)$ 
such that $\emptyset \neq(p, q) \cap E=\left(E \cap P_{n}\right) \cap(p, q)$. We may suppose without loss of generality that $p, q \in E$ and $[p, q] \cap E$ is perfect. Applying Lemma 4.1 to $[p, q] \cap E$ and $\delta_{n}$, there exists a finite set $\pi=\{([x, y], t): t \in\{x, y\} \subset E$, $t$ is a limit point of $[x, y] \cap E$, and $\{[x, y]\}$ are nonoverlapping intervals $\}$ and $\cup_{([x, y], t) \in \pi}[x, y] \supseteq E$. Clearly $\pi$ is a $\beta$ - partial partition of $[a, b]$. Since $[p, q] \backslash \cup_{([x, y], t) \in \pi}[x, y]$ consists of a finite number of intervals contiguous to $E$, it follows that $[p, q]$ admits a $\beta$ - partition. Similarly it follows that each $\left[p_{1}, q_{1}\right]$ admits such a partition, whenever $\left(p_{1}, q_{1}\right) \subset(p, q)$. Therefore $(p, q) \in \mathcal{A}$.

By (i)-(iv) and the Romanovski Lemma, it follows that $(a, b) \in \mathcal{A}$.

The second part follows by Remark 4.1, (i).

Remark 4.2. Lemma 4.2 generalizes a result of Henstock [7, p. 56] as well as Theorem 3.1 of Lee and Soedijono [12, p. 265].

\section{A Characterization of ACG $\cap \mathcal{C}$ on a Real Compact Set}

Definition 5.1. Let $F:[a, b] \rightarrow \mathbb{R}$ and $P \subseteq[a, b]$. $F$ is said to be $N_{\mathcal{B}_{o}}$ on $P$ if it has the following property: for every $\epsilon>0$ and every $Z \subset P,|Z|=0$, there exists a $\beta=\beta\left(\left\{Z_{i}\right\},\left\{\delta_{i}\right\}\right) \in \mathcal{B}_{Z} \in \mathcal{B}_{o}$, such that $\sum_{([x, y], t) \in \pi}|F(y)-F(x)|<\epsilon$, whenever $\pi$ is a $\beta$-partial partition of $Z$.

Lemma 5.1. Let $F_{1}, F_{2}:[a, b] \rightarrow \mathbb{R}$ and $P \subseteq[a, b]$. If $F_{1}, F_{2} \in N_{\mathcal{B}_{o}}$ on $P$ and $\alpha_{1}, \alpha_{2} \in \mathbb{R}$ then $\alpha_{1} F_{1}+\alpha_{2} F_{2} \in N_{\mathcal{B}_{o}}$ on $P$.

Proof. Clearly $\alpha_{1} F_{1}$ and $\alpha_{2} F_{2}$ are $N_{\mathcal{B}_{o}}$ on $P$, so it is sufficient to prove that $F_{1}+F_{2} \in N_{\mathcal{B}_{o}}$ on $P$. Let $\epsilon>0$ and $Z \subset P,|Z|=0$. Since $F_{k} \in N_{\mathcal{B}_{o}}, k=$ 1,2 , there exists $\beta_{k}=\beta_{k}\left(\left\{Z_{i}^{(k)}\right\},\left\{\delta_{i}^{(k)}\right\}\right) \in \mathcal{B}_{Z}$ such that $\sum_{([x, y], t) \in \pi} \mid F_{k}(y)-$

$F_{k}(x) \mid<\epsilon / 2$, whenever $\pi$ is a $\beta_{k}$-partial partition of $Z$. Let $Z_{i j}=Z_{i}^{(1)} \cap Z_{j}^{(2)}$. Then $\left\{Z_{i j}\right\}_{i, j} \in \mathcal{P}_{Z}$. Let $\delta_{i j}: Z_{i j} \rightarrow(0, \infty), \delta_{i j}(x)=\min \left\{\delta_{i}^{(1)}(x), \delta_{j}^{(2)}(x)\right\}$. Then $\beta=\beta\left(\left\{Z_{i j}\right\},\left\{\delta_{i j}\right\}\right) \in \mathcal{B}_{Z}$. Let $\pi$ be a $\beta$-partial partition of $Z$. Clearly $\pi$ is also a $\beta_{1}$ and a $\beta_{2}$-partial partition of $Z$. It follows that $\sum_{([x, y], t) \in \pi} \mid\left(F_{1}+\right.$ $\left.F_{2}\right)(y)-\left(F_{1}+F_{2}\right)(x)\left|\leq \sum_{([x, y], t) \in \pi}\right| F_{1}(y)-F_{1}(x)\left|+\sum_{([x, y], t) \in \pi}\right| F_{2}(y)-$ $F_{2}(x) \mid<\epsilon$. Therefore $F_{1}+F_{2} \in N_{\mathcal{B}_{o}}$ on $P$.

Lemma 5.2. Let $F:[a, b] \rightarrow \mathbb{R}$ and $Z \subset[a, b]$ such that $|Z|=0$. If $F \in V B G$ on $Z$ and $|F(Z)|>0$ then there exists $Z_{o} \subset Z$ such that $F$ is bounded and strictly monotone on $Z_{o}$, and $\left|F\left(Z_{o}\right)\right|>0$.

Proof. Since $|F(Z)|>0$ and $F \in V B G$ on $Z$ it follows that $Z=\cup_{i=1}^{\infty} A_{i}$, such that $F \in V B$ on each $A_{i}$ and $\left|F\left(A_{i}\right)\right|>0$ for at least one $A_{i}$. Therefore we may suppose without loss of generality that $F \in V B$ on $Z$. Let 


$$
\begin{aligned}
& A=\left\{x \in Z:\left(F_{/ Z}\right)^{\prime}(x) \text { exists and is finite }\right\} \\
& B=\left\{x \in Z:\left(F_{/ Z}\right)^{\prime}(x) \text { does not exist, finite or infinite }\right\} \\
& C=\left\{x \in Z:\left(F_{/ Z}\right)^{\prime}(x)= \pm \infty\right\} .
\end{aligned}
$$

Clearly $Z=A \cup B \cup C$. Let $\tilde{F}:[a, b] \rightarrow \mathbb{R}$ such that $\tilde{F} \in V B$ on $[a, b]$ and $\tilde{F}=F$ on $Z$ (this is possible, see for example [1, p. 42]). Then $\tilde{F}^{\prime}(x)$ does not exist on $B$. By Theorem 7.2 of $[15$, p. 230], $|F(B)|=0$. (That $|F(B)|=0$ follows also directly from Theorem 4.4 of $[15$, p. 223].)

We show that $F$ is $L G$ on $A$ (a function $F$ is said to be $L G$ on a set $A$ if $A=\cup_{n} A_{n}$ and $F$ is Lipschitz on each $A_{n}$, see [1]). Let $A_{n}=\{x \in A$ : $\left.\left|\left(F_{/ Z}\right)^{\prime}(x)\right|<n\right\}$. Then $A=\cup_{n=1}^{\infty} A_{n}$. For $x \in A_{n}$ there exists $\delta(x)>0$ such that $|(F(y)-F(x)) /(y-x)|<n$, whenever $y \neq x, y \in Z \cap(x-\delta(x), x+\delta(x))$. Let $A_{n, j}=\left\{x \in A_{n}: \delta(x)>1 / j\right\}$ and $A_{n, j, k}=A_{n, j} \cap[a+(k-1) / j, a+k / j]$, $j=1,2, \ldots, k=0, \pm 1, \pm 2, \ldots$. Then $A_{n}=\cup_{j}\left(\cup_{k} A_{n, j, k}\right)$. If $x<y, x, y \in$ $A_{n, j, k}$ then $0<y-x<1 / j<\min \{\delta(x), \delta(y)\}$. It follows that $|F(y)-F(x)|<$ $n \cdot|y-x|$, hence $F$ is Lipschitz on $A_{n, j, k}$. Therefore $F$ is $L G$ on $A$. Since $L G \subset(N)$ (see for example Corollary 2.32.1, (iv) of [1]) and $|A|=0$, it follows that $|F(A)|=0$.

But $F(Z)=F(A) \cup F(B) \cup F(C)$, hence $|F(C)|=|F(Z)|>0$. We may suppose without loss of generality that $C=\left\{x \in Z:\left(F_{/ Z}\right)^{\prime}=+\infty\right\}$. Let $\delta: C \rightarrow(0,+\infty)$ such that $(F(y)-F(x)) /(y-x)>1$, whenever $y \neq x$ and $y \in Z \cap(x-\delta(x), x+\delta(x))$. Let

$$
\begin{aligned}
& C_{i}=\{x \in C: \delta(x)>1 / i\}, i=1,2, \ldots ; \\
& C_{i j}=C_{i} \cap[a+(j-1) / i, a+j / i], i=1,2, \ldots, j=0, \pm 1, \pm 2, \ldots .
\end{aligned}
$$

Then $C=\cup_{i} C_{i}=\cup_{i} \cup_{j} C_{i j}$. Let $x, y \in C_{i, j}, x<y$. Then $y-x \leq 1 / i<$ $\min \{\delta(x), \delta(y)\}$. It follows that $F(y)-F(x)>y-x>0$, hence $F$ is strictly increasing on $C_{i j}$. Since $F(C)=\cup_{i=1}^{\infty} \cup_{j=1}^{i} F\left(C_{i j}\right)$ and $|F(C)|>0$, it follows that there exists some $C_{i j}$ such that $\left|F\left(C_{i j}\right)\right|>0$. Let's denote this $C_{i j}$ by $Z_{o}$. Since $F$ is $V B$ on $Z_{o} \subset Z, F$ is bounded on $Z_{o}$. Thus $Z_{o}$ has the required properties.

Theorem 5.1. Let $F:[a, b] \rightarrow \mathbb{R}$ and $P \subseteq[a, b]$. If $F$ is $N_{\mathcal{B}_{o}}$ on $P$ and $Z \subset P,|Z|=0$ then

(i) F is $V B G$ on $Z$;

(ii) $|F(Z)|=0$, hence $N_{\mathcal{B}_{o}} \subset(N)$. 
Proof. (i) For $\epsilon=1$ let $\beta=\beta\left(\left\{Z_{i}\right\},\left\{\delta_{i}\right\}\right) \in \mathcal{B}_{Z}$ be such that $\sum_{([x, y], t) \in \pi} \mid F(y)$ $-F(x) \mid<1$ whenever $\pi$ is a $\beta$-partial partition of $Z$. Let $Z_{i, j}=\left\{x \in Z_{i}\right.$ : $\left.\delta_{i}(x)>1 / j\right\}$ and $Z_{i, j, k}=Z_{i, j} \cap[a+(k-1) / j, a+k / j], j=1,2, \ldots, k=$ $0, \pm 1, \pm 2, \ldots$ Then $Z_{i}=\cup_{j} Z_{i, j}=\cup_{j}\left(\cup_{k} Z_{i, j, k}\right)$. Let $\left\{\left[\alpha_{n}, \beta_{n}\right]\right\}, n=1,2, \ldots, p$ be a finite set of nonoverlapping closed intervals, with endpoints in $Z_{i, j, k}$. Since $0<\beta_{n}-\alpha_{n}<1 / j<\min \left\{\delta_{i}\left(\alpha_{n}\right), \delta_{i}\left(\beta_{n}\right)\right\}$, it follows that $\left(\left[\alpha_{n}, \beta_{n}\right], \alpha_{n}\right) \in \beta$, hence $\sum_{n=1}^{p}\left|F\left(\beta_{n}\right)-F\left(\alpha_{n}\right)\right|<1$. Therefore $F$ is $V B G$ on the set $Z$.

(ii) Suppose on the contrary that $|F(Z)|>0$. By (i) and Lemma 5.2, it follows that there exists $Z_{o} \subset Z$ such that $F$ is strictly increasing (for example) and bounded on $Z_{o}$, and $\left|F\left(Z_{o}\right)\right|>0$. Let $\epsilon=\left|F\left(Z_{o}\right)\right|$. For $\epsilon / 4$ there exists a $\beta=\beta\left(\left\{Z_{i}\right\},\left\{\delta_{i}\right\}\right) \in \mathcal{B}_{Z_{o}}$ such that $\sum_{([x, y], t) \in \pi}|F(y)-F(x)|<\epsilon / 4$, whenever $\pi$ is a $\beta$-partial partition of $Z_{o}$. Since $F$ is $V B$ on $Z_{o}$, it follows that $F_{/ Z_{o}}$ is continuous nearly everywhere on $Z_{o}$. We may suppose without loss of generality that $F_{/ Z_{o}}$ is continuous on $Z_{o}$ (because $\left|F\left(Z_{o}\right)\right|>0$ ). Since each $Z_{i}$ contains countable many isolated points of $Z_{i}$ (see $[15$, p. 260]), we may suppose without loss of generality that $Z_{i}$ contains no isolated points of $Z_{i}$. Let $Y_{1}=Z_{1}, Y_{1}=Z_{i} \backslash\left(\cup_{j=1}^{i-1} Z_{j}\right), i \geq 2$. Then $Z_{o}=\cup_{i=1}^{\infty} Y_{i}$ and $Y_{i_{1}} \cap Y_{i_{2}}=\emptyset$ for $i_{1} \neq i_{2}$. Let $t \in Z_{o}$. Then there exists an unique $i$ such that $t \in Y_{i}$. Let

$$
\mathcal{A}_{i}=\{\langle F(t), F(x)\rangle\}_{\substack{(\langle t, x\rangle, t) \in \beta\left(Z_{i}, \delta_{i}\right) \\ t \in Y_{i}}}
$$

and $\mathcal{A}=\{\langle F(x), F(y)\rangle\}_{(\langle x, y\rangle, x) \in \beta}$. Then $\mathcal{A}$ is a Vitali cover for $F\left(Z_{o}\right)$ (indeed: if $x_{o} \in Z_{o}$ then $x \in Z_{i}$ for some $i$, and for each

$$
y \in\left(\left(x-\delta_{i}(x), x+\delta_{i}(x)\right) \cap Z_{i}\right) \backslash\{x\} \neq \emptyset
$$

we have $\langle F(x), F(y)\rangle \in \mathcal{A}, F(x) \neq F(y)$; if $y \rightarrow x$ then $F(y) \rightarrow F(x)$ ). Let $\pi \subset \beta$ be a finite subset such that

$$
\sum_{(\langle x, y\rangle, x) \in \pi}|F(y)-F(x)|>\frac{3}{4}\left|F\left(Z_{o}\right)\right|
$$

and $\{\langle F(x), F(y)\rangle\}_{(\langle x, y\rangle, x) \in \pi}$ contains only pairwise disjoint closed intervals (by Vitali's Covering Theorem - see for example [1, p. 10]). Since $F$ is strictly increasing, $\pi$ is a partition, so

$$
\frac{3}{4}\left|F\left(Z_{o}\right)\right|<\sum_{(\langle x, y\rangle, x) \in \pi}|F(y)-F(x)|<\frac{\epsilon}{4}=\frac{1}{4}\left|F\left(Z_{o}\right)\right|,
$$

a contradiction. 
Lemma 5.3. ([1, p. 12]) Let $P$ be a closed subset of $[a, b]$ and let $F \in \mathcal{C}$ on $P$. The following assertions are equivalent:

(i) $F \in V B G$ on $P$;

(ii) For every closed subset $S$ of $P$ there exists $(\alpha, \beta) \cap S \neq \emptyset$ such that $F$ is $V B$ on $(\alpha, \beta)$.

(iii) $F \in V B G$ on $Z$ whenever $Z \subset P$ and $|Z|=0$.

Remark 5.1. (i) $\Leftrightarrow$ (ii) in Lemma 5.3 follows also by [15, p. 223].

Lemma 5.4. Let $F:[a, b] \rightarrow \mathbb{R}$ and $P \subset[a, b]$. If $F \in A C G$ on $P$ then $F \in N_{\mathcal{B}_{o}}$ on $P$.

Proof. Let $\epsilon>0$ and $Z \subset P,|Z|=0$. Since $F$ is $A C G$ on $P, F$ is $A C G$ on $Z$ too. So there exists a sequence of sets $\left\{Z_{i}\right\}_{i}$ such that $Z=\cup_{i=1}^{\infty} Z_{i}$ and $F$ is $A C$ on each $Z_{i}$. For $\epsilon / 2^{i}$ let $\eta_{i}>0$ be given by the fact that $F$ is $A C$ on $Z_{i}$, and let $G_{i}$ be an open set such that $Z_{i} \subset G_{i}$ and $\left|G_{i}\right|<\eta_{i}$. Further, let $\delta_{i}: Z_{i} \rightarrow(0,+\infty)$ such that $\left(x-\delta_{i}(x), x+\delta_{i}(x)\right) \subset G_{i}$, for every $x \in Z_{i}$; let $\beta=\beta\left(\left\{Z_{i}\right\},\left\{\delta_{i}\right\}\right)$ and let $\pi$ be a $\beta$-partial partition of $Z$. We denote by $\pi_{i}=\left\{([x, y], t) \in \pi: t \in Z_{i}\right\}$. Clearly $x, y \in Z_{i}$ and $[x, y] \subset G_{i}$, whenever $([x, y], t) \in \pi_{i}$. It follows that

$$
\sum_{([x, y], t) \in \pi}|F(y)-F(x)|=\sum_{i=1}^{\infty} \sum_{([x, y], t) \in \pi_{i}}|F(y)-F(x)|<\sum_{i=1}^{\infty} \frac{\epsilon}{2^{i}}=\epsilon,
$$

hence $F \in N_{\mathcal{B}_{o}}$ on $P$.

Theorem 5.2. Let $P$ be a closed subset of $[a, b]$ and let $F \in \mathcal{C}$ on $P$. The following assertions are equivalent:

(i) $F \in A C G$ on $P$;

(ii) $F \in N_{\mathcal{B}_{o}}$ on $P$.

Proof. (i) $\Rightarrow$ (ii) See Lemma 5.4

(ii) $\Rightarrow$ (i) By Theorem 5.1, $F$ is $(N)$ on $P$, and $F$ is $V B G$ on every $Z \subset P$, with $|Z|=0$. By Lemma 5.3 , it follows that $F$ is $V B G$ on $P$. Therefore $F \in V B G \cap(N) \cap \mathcal{C}=A C G \cap \mathcal{C}$ (see for example [1, p. 75]). 


\section{$6 \quad$ The Lusin Type $\left[\mathcal{S}_{1} \mathcal{S}_{2} \mathcal{D}\right]$ Integral}

Definition 6.1. Let $\mathcal{S}_{1}=\left\{\mathcal{S}_{1}(x)\right\}_{x \in \mathbb{R}}$ be a local system $\mathcal{S}_{\infty}^{+}$-filtering on $[a, b)$, and let $\mathcal{S}_{2}=\left\{\mathcal{S}_{2}(x)\right\}_{x \in \mathbb{R}}$ be a local system $\mathcal{S}_{\infty}^{-}$-filtering on $(a, b]$. Let $f$ : $[a, b] \rightarrow \overline{\mathbb{R}}$. $f$ is said to be $\left[\mathcal{S}_{1} \mathcal{S}_{2} \mathcal{D}\right]$-integrable on $[a, b]$, if there exists $F:[a, b] \rightarrow$ $\mathbb{R}$ such that $F$ is $\left(\mathcal{S}_{1} ; \mathcal{S}_{2}\right) \mathcal{C}$ on $[a, b], F \in[A C G]$ on $[a, b]$ and $F_{a p}^{\prime}(x)=f(x)$ a.e. on $[a, b]$. We write $\left[\mathcal{S}_{1} \mathcal{S}_{2} \mathcal{D}\right] \int_{a}^{x} f(t) d t=F(x)-F(a)$.

Lemma 6.1. The $\left[\mathcal{S}_{1} \mathcal{S}_{2} \mathcal{D}\right]$-integral is well defined.

Proof. Let $F, G:[a, b] \rightarrow \mathbb{R}, F, G \in[A C G] \cap\left(\mathcal{S}_{1} ; \mathcal{S}_{2}\right) \mathcal{C}$ on $[a, b]$ such that $F_{a p}^{\prime}(x)=G_{a p}^{\prime}(x)=f(x)$ a.e. on $[a, b]$. Then $(F-G)_{a p}^{\prime}(x)=0$ a.e. on $[a, b]$ and $F-G \in[A C G] \cap\left(\mathcal{S}_{\infty}^{+} ; \mathcal{S}_{\infty}^{-}\right) \mathcal{C}$ on $[a, b]$. By Corollary $2.1, F-G$ is constant on $[a, b]$.

Remark 6.1. We have the following special cases for the $\left[\mathcal{S}_{1} \mathcal{S}_{2} \mathcal{D}\right]$-integral:

- The $\left[\mathcal{S}_{o}^{+} \mathcal{S}_{o}^{-} \mathcal{D}\right]$-integral is in fact the wide Denjoy integral $\mathcal{D}$. Therefore any $\left[\mathcal{S}_{1} \mathcal{S}_{2} \mathcal{D}\right]$-integral contains the $\mathcal{D}$-integral.

- The $\left[\mathcal{S}_{a p}^{+} \mathcal{S}_{a p}^{-} \mathcal{D}\right]$-integral is in fact the $\beta$-Ridder integral (that is also called by Kubota the $A D$ integral, see [14], [8]).

- For $\alpha, \beta \in(1 / 2,1)$ we obtain the $\left[\mathcal{S}_{\alpha}^{+} \mathcal{S}_{\beta}^{-} \mathcal{D}\right]$-integral, that seems to be new.

- The $\left[\mathcal{S}_{\text {pro }}^{+} \mathcal{S}_{\text {pro }}^{-} \mathcal{D}\right]$-integral is strictly contained in many of the integrals studied by Sarkhel, De and Kar in [18], [16], [17], [19].

Lemma 6.2. Let $f:[a, b] \rightarrow \overline{\mathbb{R}}$ be $\left[\mathcal{S}_{1} \mathcal{S}_{2} \mathcal{D}\right]$-integrable on $[a, b]$, and let $c \in$ $(a, b)$. Then $f$ is $\left[\mathcal{S}_{1} \mathcal{S}_{2} \mathcal{D}\right]$-integrable on both $[a, c]$ and $[c, b]$, and we have

$$
\left[\mathcal{S}_{1} \mathcal{S}_{2} \mathcal{D}\right] \int_{a}^{b} f(t) d t=\left[\mathcal{S}_{1} \mathcal{S}_{2} \mathcal{D}\right] \int_{a}^{c} f(t) d t+\left[\mathcal{S}_{1} \mathcal{S}_{2} \mathcal{D}\right] \int_{c}^{b} f(t) d t
$$

Proof. Let $F(x)=\left[\mathcal{S}_{1} \mathcal{S}_{2} \mathcal{D}\right] \int_{a}^{x} f(t) d t$ and let

$$
\begin{aligned}
& F_{1}:[a, c] \rightarrow \mathbb{R}, F_{1}(x)=F(x) \text { if } x \in[a, c] ; \\
& F_{2}:[c, b] \rightarrow \mathbb{R}, F_{2}(x)=F(x) \text { if } x \in[c, b] .
\end{aligned}
$$

By Lemma $2.2, F_{1}, F_{2} \in\left(\mathcal{S}_{1} ; \mathcal{S}_{2}\right) \mathcal{C}$ on $[a, c]$ respectively $[c, b]$. Then $f$ is $\left[\mathcal{S}_{1} \mathcal{S}_{2} \mathcal{D}\right]$-integrable on $[a, c]$ and on $[c, b]$, and we have

$$
\left[\mathcal{S}_{1} \mathcal{S}_{2} \mathcal{D}\right] \int_{a}^{c} f(t) d t=F_{1}(c)-F_{1}(a)=F(c)-F(a) ;
$$




$$
\left[\mathcal{S}_{1} \mathcal{S}_{2} \mathcal{D}\right] \int_{c}^{b} f(t) d t=F_{2}(b)-F_{2}(c)=F(b)-F(c) .
$$

Now (2) follows immediately.

Lemma 6.3. Let $f:[a, b] \rightarrow \overline{\mathbb{R}}$ be $\left[\mathcal{S}_{1} \mathcal{S}_{2} \mathcal{D}\right]$-integrable on $[a, c]$ and on $[c, b]$, where $c \in(a, b)$. Then $f$ is $\left[\mathcal{S}_{1} \mathcal{S}_{2} \mathcal{D}\right]$-integrable on $[a, b]$ and

$$
\left[\mathcal{S}_{1} \mathcal{S}_{2} \mathcal{D}\right] \int_{a}^{b} f(t) d t=\left[\mathcal{S}_{1} \mathcal{S}_{2} \mathcal{D}\right] \int_{a}^{c} f(t) d t+\left[\mathcal{S}_{1} \mathcal{S}_{2} \mathcal{D}\right] \int_{c}^{b} f(t) d t
$$

Proof. Let $F_{1}:[a, c] \rightarrow \mathbb{R}, F_{1} \in[A C G] \cap\left(\mathcal{S}_{1} \mathcal{S}_{2}\right) \mathcal{C}$ such that $\left(F_{1}\right)_{a p}^{\prime}(x)=f(x)$ a.e. on $[a, c]$. Let $F_{2}:[c, b] \rightarrow \mathbb{R}, F_{2} \in[A C G] \cap\left(\mathcal{S}_{1} \mathcal{S}_{2}\right) \mathcal{C}$ such that $\left(F_{2}\right)_{a p}^{\prime}(x)=$ $f(x)$ a.e. on $[c, b]$. Let $F:[a, b] \rightarrow \mathbb{R}$,

$$
F(x)= \begin{cases}F_{1}(x) & , \quad x \in[a, c] \\ F_{2}(x)+F_{1}(c)-F_{2}(c) & , x \in[c, b]\end{cases}
$$

Then $F \in[A C G] \cap\left(\mathcal{S}_{1} ; \mathcal{S}_{2}\right) \mathcal{C}$ on $[a, b]$ (see Lemma 2.2) and $F_{a p}^{\prime}(x)=f(x)$ a.e. on $[a, b]$. Hence $f$ is $\left[\mathcal{S}_{1} \mathcal{S}_{2} \mathcal{D}\right]$-integrable on $[a, b]$ and we have $(3)$.

Lemma 6.4. Let $f_{1}, f_{2}:[a, b] \rightarrow \overline{\mathbb{R}}$ be $\left[\mathcal{S}_{1} \mathcal{S}_{2} \mathcal{D}\right]$-integrable on $[a, b]$, and let $\alpha_{1}, \alpha_{2} \in \mathbb{R}$. If $\mathcal{S}_{1}$ is filtering on $[a, b)$ and $\mathcal{S}_{2}$ is filtering on $(a, b]$, then $\alpha_{1} f_{1}+$ $\alpha_{2} f_{2}$ is $\left[\mathcal{S}_{1} \mathcal{S}_{2} \mathcal{D}\right]$-integrable on $[a, b]$ and $\left[\mathcal{S}_{1} \mathcal{S}_{2} \mathcal{D}\right] \int_{a}^{b}\left(\alpha_{1} f_{1}+\alpha_{2} f_{2}\right)(t) d t=\alpha_{1}$. $\left[\mathcal{S}_{1} \mathcal{S}_{2} \mathcal{D}\right] \int_{a}^{b} f_{1}(t) d t+\alpha_{2} \cdot\left[\mathcal{S}_{1} \mathcal{S}_{2} \mathcal{D}\right] \int_{a}^{b} f_{2}(t) d t$.

Proof. Since $f_{1}$ and $f_{2}$ are $\left[\mathcal{S}_{1} \mathcal{S}_{2} \mathcal{D}\right]$-integrable on $[a, b]$, there exist $F_{1}, F_{2}$ : $[a, b] \rightarrow \mathbb{R}$, belonging to $[A C G] \cap\left(\mathcal{S}_{1} ; \mathcal{S}_{2}\right) \mathcal{C}$ on $[a, b]$, such that $\left(F_{1}\right)_{a p}^{\prime}(x)=f_{1}(x)$ and $\left(F_{2}\right)_{a p}^{\prime}(x)=f_{2}(x)$ a.e. on $[a, b]$. Clearly $\left(\alpha_{1} F_{1}+\alpha_{2} F_{2}\right)_{a p}^{\prime}(x)=\alpha_{1} f_{1}(x)+$ $\alpha_{2} f_{2}(x)$ a.e. on $[a, b]$, and $\alpha_{1} F_{1}+\alpha_{2} F_{2} \in[A C G]$ on $[a, b]$. Then $\alpha_{1} F_{1}+\alpha_{2} F_{2}$ is left $\mathcal{S}_{1} \mathcal{C}$ on $(a, b]$ (since $\mathcal{S}_{1}$ is filtering on $\left.(a, b]\right)$ and $\alpha_{1} F_{1}+\alpha_{2} F_{2}$ is right $\mathcal{S}_{2} \mathcal{C}$ on $(a, b]$ (since $\mathcal{S}_{2}$ is filtering on $[a, b)$ ). By Lemma 2.1 it follows that $\alpha_{1} F_{1}+\alpha_{2} F_{2}$ is $\left(\mathcal{S}_{1} ; \mathcal{S}_{2}\right) \mathcal{C}$ on $[a, b]$. Thus $\alpha_{1} f_{1}+\alpha_{2} f_{2}$ is $\left[\mathcal{S}_{1} \mathcal{S}_{2} \mathcal{D}\right]$-integrable on $[a, b]$ and $\left[\mathcal{S}_{1} \mathcal{S}_{2} \mathcal{D}\right] \int_{a}^{b}\left(\alpha_{1} f_{1}+\alpha_{2} f_{2}\right)(t) d t=\left(\alpha_{1} F_{1}+\alpha_{2} F_{2}\right)(b)-\left(\alpha_{1} F_{1}+\right.$ $\left.\alpha_{2} F_{2}\right)(a)=\alpha_{1} \cdot\left[\mathcal{S}_{1} \mathcal{S}_{2} \mathcal{D}\right] \int_{a}^{b} f_{1}(t) d t+\alpha_{2} \cdot\left[\mathcal{S}_{1} \mathcal{S}_{2} \mathcal{D}\right] \int_{a}^{b} f_{2}(t) d t$.

\section{$7 \quad$ The Riemann Type $\left[\mathcal{S}_{1} \mathcal{S}_{2} \mathcal{R}\right]$ Integral}

Definition 7.1. Let $\mathcal{S}_{1}=\left\{\mathcal{S}_{1}(x)\right\}_{x \in \mathbb{R}}$ be a local system $\mathcal{S}_{\infty}^{+}$-filtering on $[a, b)$, and let $\mathcal{S}_{2}=\left\{\mathcal{S}_{2}(x)\right\}_{x \in \mathbb{R}}$ be a local system $\mathcal{S}_{\infty}^{-}$-filtering on $(a, b]$. Let $f$ : $[a, b] \rightarrow \mathbb{R} . f$ is said to be $\left[\mathcal{S}_{1} \mathcal{S}_{2} \mathcal{R}\right]$ integrable on $[a, b]$, if there is a real number 
$I$ with the following property: for every $\epsilon>0$ there exists $\beta=\beta\left(\left\{X_{i}\right\},\left\{\delta_{i}\right\}\right) \in$ $\overline{\mathcal{B}}_{[a, b]}$ such that for every countable set $A$ with $A \supset I s\left(\left\{X_{i}\right\}\right)$ there exists $\beta_{A}=\beta_{A}\left(\sigma_{x}^{(1)}, \sigma_{x}^{(2)}\right) \in \mathcal{B}_{A}\left(\mathcal{S}_{1}, \mathcal{S}_{2}\right)$ so that $|s(f, \pi)-I|<\epsilon / 2$ whenever $\pi$ is a $\left(\beta \cup \beta_{A}\right)$-partition of $[a, b]$.

Theorem 7.1. The number I in Definition 7.1 is unique, and we denote it by $\left[\mathcal{S}_{1} \mathcal{S}_{2} \mathcal{R}\right] \int_{a}^{b} f(t) d t$.

Proof. Suppose that there exist two numbers $I_{1}$ and $I_{2}$ as in Definition 7.1. For $\epsilon>0$ and $I_{k}, k=1,2$ let $\beta_{k}=\beta_{k}\left(\left\{X_{i}^{(k)}\right\},\left\{\delta_{i}^{(k)}\right\}\right)$ be given by Definition 7.1. For $A=I s\left(\left\{X_{i}^{(1)}\right\}\right) \cup I s\left(\left\{X_{i}^{(2)}\right\}\right)$ let

$$
\beta_{A}^{(k)}=\beta_{A}^{(k)}\left(\sigma_{x}^{(k, 1)}, \sigma_{x}^{(k, 2)}\right) \in \mathcal{B}_{A}\left(\mathcal{S}_{1} ; \mathcal{S}_{2}\right),
$$

$k=1,2$ be given by Definition 7.1. We define

- $\sigma_{x}^{(1)}=\sigma_{x}^{(1,1)} \cap \sigma_{x}^{(2,1)} \in \mathcal{S}_{\infty}^{+}(x)$;

- $\sigma_{x}^{(2)}=\sigma_{x}^{(1,2)} \cap \sigma_{x}^{(2,2)} \in \mathcal{S}_{\infty}^{-}(x)$;

- $\delta_{i, j}: X_{i} \cap X_{j} \rightarrow(0,+\infty), \delta_{i, j}(x)=\min \left\{\delta_{i}^{(1)}(x), \delta_{j}^{(2)}(x)\right\}$;

- $\beta_{3}=\beta_{3}\left(\left\{X_{i}^{(1)} \cap X_{j}^{(2)}\right\},\left\{\delta_{i, j}\right\}\right) \in \overline{\mathcal{B}}_{[a, b]}$;

- $\beta_{A}=\beta_{A}\left(\sigma_{x}^{(1)}, \sigma_{x}^{(2)}\right) \in \mathcal{B}\left(\mathcal{S}_{\infty}^{+} ; \mathcal{S}_{\infty}^{-}\right)$;

- $\beta=\beta_{3} \cup \beta_{A}$;

By Lemma 4.2 there exists $\pi$, an $\beta$-partition of $[a, b]$. Clearly $\pi$ is also a $\left(\beta_{k} \cup \beta_{A}^{(k)}\right)$-partition of $[a, b], k=1,2$. It follows that $\left|s(f, \pi)-I_{k}\right|<\epsilon$, $k=1,2$, hence $\left|I_{1}-I_{2}\right|<2 \epsilon$. Since $\epsilon$ is arbitrary we obtain that $I_{1}=I_{2}$.

Lemma 7.1. Let $f_{1}, f_{2}:[a, b] \rightarrow \mathbb{R}$ be $\left[\mathcal{S}_{1} \mathcal{S}_{2} \mathcal{R}\right]$-integrable on $[a, b]$, and let $\alpha_{1}, \alpha_{2} \in \mathbb{R}$. If $\mathcal{S}_{1}$ is filtering on $[a, b)$ and $\mathcal{S}_{2}$ is filtering on $(a, b]$, then $\alpha_{1} f_{1}+$ $\alpha_{2} f_{2}$ is $\left[\mathcal{S}_{1} \mathcal{S}_{2} \mathcal{R}\right]$ - integrable on $[a, b]$ and $\left[\mathcal{S}_{1} \mathcal{S}_{2} \mathcal{R}\right] \int_{a}^{b}\left(\alpha_{1} f_{1}+\alpha_{2} f_{2}\right)(t) d t=\alpha_{1}$. $\left[\mathcal{S}_{1} \mathcal{S}_{2} \mathcal{R}\right] \int_{a}^{b} f_{1}(t) d t+\alpha_{2} \cdot\left[\mathcal{S}_{1} \mathcal{S}_{2} \mathcal{R}\right] \int_{a}^{b} f_{2}(t) d t$.

Proof. Let $I=\left[\mathcal{S}_{1} \mathcal{S}_{2} \mathcal{R}\right] \int_{a}^{b} f_{1}(t) d t$ and $J=\left[\mathcal{S}_{1} \mathcal{S}_{2} \mathcal{R}\right] \int_{a}^{b} f_{2}(t) d t$. Suppose that $\alpha_{1}, \alpha_{2} \neq 0$ and let $\epsilon>0$.

For $\epsilon /\left(2\left|\alpha_{2}\right|\right)$ let $\beta_{1}=\beta_{1}\left(\left\{X_{i}^{(1)}\right\},\left\{\delta_{i}^{(1)}\right\}\right) \in \overline{\mathcal{B}}_{[a, b]}$ be given by the fact that $f_{1}$ is $\left[\mathcal{S}_{1} \mathcal{S}_{2} \mathcal{R}\right]$-integrable on $[a, b]$. 
For $\epsilon /\left(2\left|\alpha_{1}\right|\right)$ let $\beta_{2}=\beta_{1}\left(\left\{X_{i}^{(2)}\right\},\left\{\delta_{i}^{(2)}\right\}\right) \in \overline{\mathcal{B}}_{[a, b]}$ be given by the fact that $f_{2}$ is $\left[\mathcal{S}_{1} \mathcal{S}_{2} \mathcal{R}\right]$-integrable on $[a, b]$.

Let $X_{i j}=X_{i}^{(1)} \cap X_{j}^{(2)}$. Then $\left\{X_{i j}\right\}_{i, j} \in \overline{\mathcal{P}}_{[a, b]}$.

Let $\delta_{i j}: X_{i j} \rightarrow(0,+\infty), \delta_{i j}(x)=\min \left\{\delta_{i}^{(1)}(x), \delta_{j}^{(2)}(x)\right\}$.

Let $\beta=\beta\left(\left\{X_{i j}\right\},\left\{\delta_{i j}\right\}\right) \in \overline{\mathcal{B}}_{[a, b]}$.

Clearly $I s\left(\left\{X_{i j}\right\}\right)$ contains both, $I s\left(\left\{X_{i}^{(1)}\right\}\right)$ and $I s\left(\left\{X_{j}^{(2)}\right\}\right)$.

Let $A$ be a countable subset of $[a, b]$ that contains $I s\left(\left\{X_{i j}\right\}\right)$.

For $\epsilon /\left(2\left|\alpha_{2}\right|\right)$, let $\beta_{A}^{(1)}=\beta_{A}^{(1)}\left(\sigma_{x}^{(1,1)}, \sigma_{x}^{(1,2)}\right) \in \mathcal{B}_{A}\left(\mathcal{S}_{1} ; \mathcal{S}_{2}\right)$ be given by the fact that $f_{1}$ is $\left[\mathcal{S}_{1} \mathcal{S}_{2} \mathcal{R}\right]$-integrable on $[a, b]$.

For $\epsilon /\left(2\left|\alpha_{1}\right|\right)$, let $\beta_{A}^{(2)}=\beta_{A}^{(2)}\left(\sigma_{x}^{(2,1)}, \sigma_{x}^{(2,2)}\right) \in \mathcal{B}_{A}\left(\mathcal{S}_{1} ; \mathcal{S}_{2}\right)$ be given by the fact that $f_{2}$ is $\left[\mathcal{S}_{1} \mathcal{S}_{2} \mathcal{R}\right]$-integrable on $[a, b]$.

Let $\sigma_{x}^{(1)}=\sigma_{x}^{(1,1)} \cap \sigma_{x}^{(2,1)}$. Since $\mathcal{S}_{1}$ is filtering, $\sigma_{x}^{(1)} \in \mathcal{S}_{1}(x)$.

Let $\sigma_{x}^{(2)}=\sigma_{x}^{(1,2)} \cap \sigma_{x}^{(2,2)}$. Since $\mathcal{S}_{2}$ is filtering, $\sigma_{x}^{(2)} \in \mathcal{S}_{2}(x)$.

Let $\beta_{A}=\beta_{A}\left(\sigma_{x}^{(1)}, \sigma_{x}^{(2)}\right) \in \mathcal{B}_{A}\left(\mathcal{S}_{1} ; \mathcal{S}_{2}\right)$.

Let $\pi$ be a $\left(\beta \cup \beta_{A}\right)$-partition of $[a, b]$. Then $\pi$ is also a $\left(\beta_{1} \cup \beta_{A}^{(1)}\right)$-partition and a $\left(\beta_{2} \cup \beta_{A}^{(2)}\right)$-partition of $[a, b]$. Then $\left|s\left(\alpha_{1} f_{1}+\alpha_{2} f_{2} ; \pi\right)-\left(\alpha_{1} I+\alpha_{2} J\right)\right| \leq$ $\left|\alpha_{1}\right| \cdot\left|s\left(f_{1} ; \pi\right)-I\right|+\left|\alpha_{2}\right| \cdot\left|s\left(f_{2} ; \pi\right)-J\right|<\epsilon / 2+\epsilon / 2=\epsilon$. Therefore $\alpha_{1} I+\alpha_{2} J=$ $\left[\mathcal{S}_{1} \mathcal{S}_{2} \mathcal{R}\right] \int_{a}^{b}\left(\alpha_{1} f_{1}+\alpha_{2} f_{2}\right)(t) d t$

Lemma 7.2 (A Cauchy criterion). Let $\mathcal{S}_{1}=\left\{\mathcal{S}_{1}(x)\right\}_{x \in \mathbb{R}}$ be a local system $\mathcal{S}_{\infty}^{+}$-filtering on $[a, b)$, and let $\mathcal{S}_{2}=\left\{\mathcal{S}_{2}(x)\right\}_{x \in \mathbb{R}}$ be a local system $\mathcal{S}_{\infty}^{-}$-filtering on $(a, b]$. Let $f:[a, b] \rightarrow \mathbb{R}$. The following assertions are equivalent:

(i) $f$ is $\left[\mathcal{S}_{1} \mathcal{S}_{2} \mathcal{R}\right]$-integrable on $[a, b]$.

(ii) For $\epsilon>0$ there exists $\beta=\beta\left(\left\{X_{i}\right\},\left\{\delta_{i}\right\}\right) \in \overline{\mathcal{B}}_{[a, b]}$ (depending on $\epsilon$ ) and for every pair of countable subsets $A_{1}$ and $A_{2}$ of $[a, b]$ there exist $\beta_{A_{k}}=\beta_{A_{k}}\left(\sigma_{x}^{(k, 1)}, \sigma_{x}^{(k, 2)}\right) \in \mathcal{B}_{A_{k}}\left(\mathcal{S}_{1} ; \mathcal{S}_{2}\right)$ (depending only on $\epsilon$ ), such that $\left|s\left(f, \pi_{1}\right)-s\left(f, \pi_{2}\right)\right|<\epsilon$, whenever $\pi_{k}$ is a $\left(\beta \cup \beta_{A_{k}}\right)$-partition of $[a, b]$, $k=1,2$. 
Proof. (i) $\Rightarrow$ (ii) This is obvious.

(ii) $\Rightarrow$ (i) For $\epsilon_{k}=1 / k, k=\overline{2,+\infty}$, let $\beta_{k}=\beta_{k}\left(\left\{X_{i}^{(k)}\right\},\left\{\delta_{i}^{(k)}\right\}\right)$ be given by (ii). Let $A_{k}=\operatorname{Is}\left(\left\{X_{i}^{(k)}\right\}\right)$. For $n>m>2$ let $A_{m, n}=A_{m} \cup A_{n} \cup \operatorname{Is}\left(\left\{X_{i}^{(m)} \cap\right.\right.$ $\left.\left.X_{j}^{(n)}\right\}\right)$. By (ii), for $\epsilon_{m}$ and the pair of sets $A_{m}$ and $A_{m, n}$, there exist

$$
\beta_{A_{m}}=\beta_{A_{m}}\left(\sigma_{x}^{(m, 1)}, \sigma_{x}^{(m, 2)}\right) \in \mathcal{B}_{A_{m}}\left(\mathcal{S}_{1} ; \mathcal{S}_{2}\right)
$$

and

$$
\beta_{A_{m, n}}^{(1)}=\beta_{A_{m, n}}^{(1)}\left(\sigma_{x}^{(m, n, 1,1)}, \sigma_{x}^{(m, n, 1,2)}\right) \in \mathcal{B}_{A_{m, n}}\left(\mathcal{S}_{1} ; \mathcal{S}_{2}\right)
$$

such that $\left|s\left(f, \pi_{1}\right)-s\left(f, \pi_{2}\right)\right|<\epsilon_{m}$ whenever $\pi_{1}$ is a $\left(\beta_{m} \cup \beta_{A_{m}}\right)$-partition for $[a, b]$ and $\pi_{2}$ is a $\left(\beta_{m} \cup \beta_{A_{m, n}}^{(1)}\right)$-partition of $[a, b]$.

Again by (ii), for $\epsilon_{n}$ and the pair of sets $A_{n}$ and $A_{m, n}$, there exist $\beta_{A_{n}}=$ $\beta_{A_{n}}\left(\sigma_{x}^{(n, 1)}, \sigma_{x}^{(n, 2)}\right) \in \mathcal{B}_{A_{n}}\left(\mathcal{S}_{1} ; \mathcal{S}_{2}\right)$ and $\beta_{A_{m, n}}^{(2)}=\beta_{A_{m, n}}^{(2)}\left(\sigma_{x}^{(m, n, 2,1)}, \sigma_{x}^{(m, n, 2,2)}\right) \in$ $\mathcal{B}_{A_{m, n}}\left(\mathcal{S}_{1} ; \mathcal{S}_{2}\right)$ such that $\left|s\left(f, \pi_{1}\right)-s\left(f, \pi_{2}\right)\right|<\epsilon_{n}$ whenever $\pi_{1}$ is a $\left(\beta_{n} \cup \beta_{A_{n}}\right)$ partition for $[a, b]$ and $\pi_{2}$ is a $\left(\beta_{n} \cup \beta_{A_{m, n}}^{(2)}\right)$-partition of $[a, b]$. Let

$$
\begin{aligned}
& \delta_{i, j}^{(m, n)}: X_{i}^{(m)} \cap X_{j}^{(n)} \rightarrow(0,+\infty), \delta_{i, j}^{(m, n)}(x)=\min \left\{\delta_{i}^{(m)}(x), \delta_{j}^{(n)}(x)\right\} \\
& \beta_{m, n}=\beta_{m, n}\left(\left\{X_{i}^{(m)} \cap X_{j}^{(n)}\right\},\left\{\delta_{i, j}^{(m, n)}\right\}\right) \in \overline{\mathcal{B}}_{[a, b]} ; \\
& \sigma_{x}^{(m, n, 1)}=\sigma_{x}^{(m, n, 1,1)} \cap \sigma_{x}^{(m, n, 2,1)} \\
& \sigma_{x}^{(m, n, 2)}=\sigma_{x}^{(m, n, 1,2)} \cap \sigma_{x}^{(m, n, 2,2)} \\
& \beta_{A_{m, n}}^{(3)}=\beta_{A_{m, n}}^{(3)}\left(\sigma_{x}^{(m, n, 1)}, \sigma_{x}^{(m, n, 2)}\right) \in \mathcal{B}_{A_{m, n}}\left(\mathcal{S}_{\infty}^{+} ; \mathcal{S}_{\infty}^{-}\right) ; \\
& \beta=\beta_{m, n} \cup \beta_{A_{m, n}}^{(3)} .
\end{aligned}
$$

By Lemma 4.2 there exists $\pi$ a $\beta$-partition of $[a, b]$. But $\pi$ is also a $\beta_{m} \cup \beta_{A_{m, n}}^{(1)}$ partition and a $\left(\beta_{n} \cup \beta_{A_{m, n}}^{(2)}\right)$-partition of $[a, b]$. Let $\pi_{m}$ be a $\left(\beta_{m} \cup \beta_{A_{m}}\right)$ partition of $[a, b]$ and let $\pi_{n}$ be a $\left(\beta_{n} \cup \beta_{A_{n}}\right)$-partition of $[a, b]$. Then $\mid s\left(f, \pi_{m}\right)-$ $s(f, \pi) \mid<\epsilon_{m}$ and $\left|s\left(f, \pi_{n}\right)-s(f, \pi)\right|<\epsilon_{n}$. It follows that $\left|s\left(f, \pi_{m}\right)-s\left(f, \pi_{n}\right)\right|<$ $1 / m+1 / n<2 / m=2 \epsilon_{m}$. Therefore $\left\{s\left(f, \pi_{m}\right)\right\}_{m}$ is a Cauchy sequence. Let's denote its limit by $I$. Then $\left|s\left(f, \pi_{m}\right)-I\right| \leq 2 \epsilon_{m}$. Let $\epsilon>0$ and $m>2$ such that $3 / m<\epsilon$. Let $A$ be a countable subset of $[a, b]$. Then there exists $\beta_{A}=$ $\beta_{A}\left(\sigma_{x}^{(1)}, \sigma_{x}^{(2)}\right) \in \mathcal{B}_{A}\left(\mathcal{S}_{1} ; \mathcal{S}_{2}\right)$ such that $\left|s(f, \pi)-s\left(f, \pi_{m}\right)\right|<1 / m$ whenever $\pi$ is a $\left(\beta_{m} \cup \beta_{A}\right)$-partition of $[a, b]$. It follows that $|s(f, \pi)-I|<1 / m+2 / m<\epsilon$. Therefore $f$ is $\left(\mathcal{S}_{1} \mathcal{S}_{2} \mathcal{R}\right)$ - integrable on $[a, b]$. 
Lemma 7.3. Let $\mathcal{S}_{1}=\left\{\mathcal{S}_{1}(x)\right\}_{x \in \mathbb{R}}$ be a local system $\mathcal{S}_{\infty}^{+}$-filtering on $[a, b)$, and let $\mathcal{S}_{2}=\left\{\mathcal{S}_{2}(x)\right\}_{x \in \mathbb{R}}$ be a local system $\mathcal{S}_{\infty}^{-}$-filtering on $(a, b]$. Let $f:[a, b] \rightarrow \mathbb{R}$.

(i) If $a<c<b$ and $f$ is $\left[\mathcal{S}_{1} \mathcal{S}_{2} \mathcal{R}\right]$-integrable on $[a, c]$ and on $[c, b]$ then $f$ is $\left[\mathcal{S}_{1} \mathcal{S}_{2} \mathcal{R}\right]$-integrable on $[a, b]$ and we have

$$
\left[\mathcal{S}_{1} \mathcal{S}_{2} \mathcal{R}\right] \int_{a}^{c} f(t) d t+\left[\mathcal{S}_{1} \mathcal{S}_{2} \mathcal{R}\right] \int_{c}^{b} f(t) d t=\left[\mathcal{S}_{1} \mathcal{S}_{2} \mathcal{R}\right] \int_{a}^{b} f(t) d t
$$

(ii) If $a \leq c<d \leq b$ and $f$ is $\left[\mathcal{S}_{1} \mathcal{S}_{2} \mathcal{R}\right]$-integrable on $[a, b]$ then $f$ is $\left[\mathcal{S}_{1} \mathcal{S}_{2} \mathcal{R}\right]$ integrable on $[c, d]$.

Proof. (i) Let $I^{(1)}=\left[\mathcal{S}_{1} \mathcal{S}_{2} \mathcal{R}\right] \int_{a}^{c} f(t) d t$ and $I^{(2)}=\left[\mathcal{S}_{1} \mathcal{S}_{2} \mathcal{R}\right] \int_{c}^{b} f(t) d t$.

Consider $\epsilon>0$.

For $\frac{\epsilon}{2}$ and $I^{(1)}$ let $\beta^{(1)}=\beta^{(1)}\left(\left\{X_{i}^{(1)}\right\},\left\{\delta_{i}^{(1)}\right\}\right) \in \overline{\mathcal{B}}_{[a, c]}$ be given by Def. 7.1.

For $\frac{\epsilon}{2}$ and $I^{(2)}$ let $\beta^{(2)}=\beta^{(2)}\left(\left\{X_{i}^{(2)}\right\},\left\{\delta_{i}^{(2)}\right\}\right) \in \overline{\mathcal{B}}_{[c, b]}$ be given by Def. 7.1.

Let $A^{(1)}=\operatorname{Is}\left(\left\{X_{i}^{(1)}\right\}\right)$ and $A^{(2)}=\operatorname{Is}\left(\left\{X_{i}^{(2)}\right\}\right)$.

Note that $c \in A^{(1)} \cap A^{(2)},\left\{X_{i}^{(1)}, X_{j}^{(2)}\right\}_{i, j} \in \overline{\mathcal{P}}_{[a, b]}$ and $\operatorname{Is}\left(\left\{X_{i}^{(1)}\right\} \cup\left\{X_{j}^{(2)}\right\}\right)=$ $A^{(1)} \cup A^{(2)}$.

Let $A$ be a countable subset of $[a, b]$.

Let $A_{1}=A^{(1)} \cup(A \cap[a, c])$ and $A_{2}=A^{(2)} \cup(A \cap[c, b])$.

Clearly $A^{(1)} \subseteq A_{1}$ and $A^{(2)} \subseteq A_{2}$. By Definition 7.1, for $\epsilon / 2$ and $A_{k}, k=1,2$ there exists $\beta_{A_{k}}=\beta_{A_{k}}\left(\sigma_{x}^{(k, 1)}, \sigma_{x}^{(k, 2)}\right) \in \mathcal{B}_{A_{k}}\left(\mathcal{S}_{1} ; \mathcal{S}_{2}\right)$ such that $\left|s(f, \pi)-I^{(1)}\right|<$ $\epsilon / 2$ whenever $\pi$ is a $\left(\beta^{(1)} \cup \beta_{A_{1}}\right)$-partition of $[a, c]$, and $\left|s(f, \pi)-I^{(2)}\right|<\epsilon / 2$ whenever $\pi$ is a $\left(\beta^{(2)} \cup \beta_{A_{2}}\right)$-partition of $[c, b]$. Let $\delta_{i}^{(1) \star}: X_{i}^{(1)} \rightarrow(0,+\infty)$ and $\delta_{j}^{(2) \star}: X_{j}^{(2)} \rightarrow(0,+\infty)$ be defined as follows:

$$
\begin{aligned}
\delta_{i}^{(1) \star}(x) & = \begin{cases}\min \left\{\delta_{i}^{(1)}(x), c-x\right\} & \text { if } x<c \\
\min \left\{\delta_{i}^{(1)}(c), c-a\right\} & \text { if } x=c\end{cases} \\
\delta_{j}^{(2) \star}(x) & = \begin{cases}\min \left\{\delta_{j}^{(2)}(x), c-x\right\} & \text { if } x>c \\
\min \left\{\delta_{j}^{(1)}(c), b-c\right\} & \text { if } x=c\end{cases}
\end{aligned}
$$

Let

$$
\sigma_{x}^{(1)}= \begin{cases}\sigma_{x}^{(1,1)} \cap(2 x-c, c) \in \mathcal{S}_{1}(x) & \text { if } x \in[a, c) \cap A_{1} \\ \sigma_{x}^{(2,1)} \in \mathcal{S}_{1}(x) & \text { if } x \in[c, b) \cap A_{2}\end{cases}
$$




$$
\sigma_{x}^{(2)}= \begin{cases}\sigma_{x}^{(1,2)} \in \mathcal{S}_{2}(x) & \text { if } \in(a, c] \cap A_{1} \\ \sigma_{x}^{(2,2)} \cap(c, 2 x-c) \in \mathcal{S}_{2}(x) & \text { if } \in(c, b] \cap A_{2}\end{cases}
$$

Let $\beta_{A}=\beta_{A}\left(\sigma_{x}^{(1)}, \sigma_{x}^{(2)}\right) \subset \beta_{A_{1}} \cup \beta_{A_{2}}$;

Let $\left\{X_{k}^{\prime}\right\}_{k}$ be a relabeling of the set $\left\{X_{i}^{(1)}\right\}_{i} \cup\left\{X_{j}^{(2)}\right\}_{j}$;

Let $\delta_{k}^{\prime}: X_{k}^{\prime} \rightarrow(0,+\infty), \delta_{k}^{\prime}=\delta_{i}^{(1) \star}$ if $X_{k}^{\prime}=X_{i}^{(1)}$, and $\delta_{k}^{\prime}=\delta_{j}^{(2) \star}$ if $X_{k}^{\prime}=X_{j}^{(2)}$

$$
\beta_{o}=\beta_{o}\left(\left\{X_{k}^{\prime}\right\},\left\{\delta_{k}^{\prime}\right\}\right) \subset \beta^{(1)} \cup \beta^{(2)} .
$$

$\beta=\beta_{o} \cup \beta_{A}$.

Let $\pi$ be a $\beta$-partition of $[a, b]$. Let $([x, y], t) \in \pi$. If $t<c$ then $y<c$, and if $t>c$ then $x>c$. It follows that

$$
C=\cup_{\substack{([x, y], t) \in \pi \\ t<c}}[x, y] \subset[a, c) \text { and } D=\cup_{\substack{([x, y], t) \in \pi \\ t>c}}[x, y] \subset(c, b] .
$$

Let $x_{c}=\sup C$ and $y_{c}=\inf D$. We observe that $\left(\left[x_{c}, c\right], c\right)$ and $\left(\left[c, y_{c}\right], c\right)$ belong to $\beta$. Let $\pi_{1}=\{(I, t) \in \pi: t \leq c\}$ and $\pi_{2}=\{(I, t) \in \pi: t \geq c\}$. Then $\pi_{1}$ is a $\left(\beta^{(1)} \cup \beta_{A_{1}}\right)$-partition of $[a, c]$ and $\pi_{2}$ is a $\left(\beta^{(2)} \cup \beta_{A_{2}}\right)$-partition of $[c, b]$. We have $\left|s(f, \pi)-I^{(1)}-I^{(2)}\right|=\left|s\left(f, \pi_{1}\right)-I^{(1)}+s\left(f, \pi_{2}\right)-I^{(2)}\right|<$ $\left|s\left(f, \pi_{1}\right)-I^{(1)}\right|+\left|s\left(f, \pi_{2}\right)-I^{(2)}\right|<\epsilon$. It follows that $f$ is $\left[\mathcal{S}_{1} \mathcal{S}_{2} \mathcal{R}\right]$-integrable on $[a, b]$ and $\left[\mathcal{S}_{1} \mathcal{S}_{2} \mathcal{R}\right] \int_{a}^{b} f(t) d t=I^{(1)}+I^{(2)}$.

(ii) Let $a<c<d<b$.

For $\epsilon>0$ let $\beta=\beta\left(\left\{X_{i}\right\},\left\{\delta_{i}\right\}\right) \in \overline{\mathcal{B}}_{[a, b]}$ be given by Lemma 7.2.

Let $\beta_{1}=\beta_{1}\left(\left\{X_{i} \cap[c, d]\right\},\left\{\delta_{i / X_{i} \cap[c, d]}\right\}\right)$.

Let $A^{(1)}, A^{(2)}$ be a pair of countable subsets of $[c, d]$.

Let $\beta_{3}=\beta_{3}\left(\left\{X_{i} \cap[a, c]\right\},\left\{\delta_{i / X_{i} \cap[a, c]}\right\}\right)$.

Let $A_{3}$ be a countable subset of $[a, c]$ such that $A_{3} \supset \operatorname{Is}\left(\left\{X_{i} \cap[a, c]\right\}\right)$.

Let $\beta_{4}=\beta_{4}\left(\left\{X_{i} \cap[d, b]\right\},\left\{\delta_{i / X_{i} \cap[d, b]}\right\}\right)$.

Clearly

$$
\beta \supseteq \beta_{1} \cup \beta_{3} \cup \beta_{4}
$$

Let $A_{4}$ be a countable subset of $[d, b]$ such that $A_{4} \supset \operatorname{Is}\left(\left\{X_{i} \cap[d, b]\right\}\right)$.

Let $A_{1}=A^{(1)} \cup A_{3} \cup A_{4} \cup \operatorname{Is}\left(\left\{X_{i} \cap[c, d]\right\}\right)$ and $A_{2}=A^{(2)} \cup A_{3} \cup A_{4} \cup \operatorname{Is}\left(\left\{X_{i} \cap\right.\right.$ $[c, d]\}$ ) (both contain $\operatorname{Is}\left(\left\{X_{i}\right\}\right)$. 
For $A_{k}, k=1,2$ let $\sigma_{x}^{(k, 1)} \in \mathcal{S}_{1}(x), x \in[a, b) \cap A_{k}$ and $\sigma_{x}^{(k, 2)} \in \mathcal{S}_{2}(x)$, $x \in(a, b] \cap A_{k}$ be given by Lemma 7.2.

Let $\beta_{A_{k}}=\beta_{A_{k}}\left(\sigma_{x}^{(k, 1)}, \sigma_{x}^{(k, 2)}\right) \in \mathcal{B}_{A_{k}}\left(\mathcal{S}_{1}, \mathcal{S}_{2}\right), k=1,2$.

Let $\beta_{A_{3}}^{(k)}=\beta_{A_{3}}^{(k)}\left(\sigma_{x}^{(k, 1)}, \sigma_{x}^{(k, 2)}\right) \in \mathcal{B}_{A_{3}}\left(\mathcal{S}_{1} ; \mathcal{S}_{2}\right), k=1,2$.

Let $\beta_{A_{4}}^{(k)}=\beta_{A_{4}}^{(k)}\left(\sigma_{x}^{(k, 1)}, \sigma_{x}^{(k, 2)}\right) \in \mathcal{B}_{A_{4}}\left(\mathcal{S}_{1} ; \mathcal{S}_{2}\right), k=1,2$.

Let $\sigma_{x}^{(1)}=\sigma_{x}^{(1,1)} \cap \sigma_{x}^{(2,1)} \in \mathcal{S}_{\infty}^{+}$, if $x \in\left(A_{3} \cup A_{4}\right) \backslash\{b\}$.

Let $\sigma_{x}^{(2)}=\sigma_{x}^{(1,2)} \cap \sigma_{x}^{(2,2)} \in \mathcal{S}_{\infty}^{-}$, if $x \in\left(A_{3} \cup A_{4}\right) \backslash\{a\}$.

Let $\beta_{A_{3}}=\beta_{A_{3}}\left(\sigma_{x}^{(1)}, \sigma_{x}^{(2)}\right) \in \mathcal{B}_{A_{3}}\left(\mathcal{S}_{\infty}^{+} ; \mathcal{S}_{\infty}^{-}\right)$.

Let $\beta_{A_{4}}=\beta_{A_{4}}\left(\sigma_{x}^{(1)}, \sigma_{x}^{(2)}\right) \in \mathcal{B}_{A_{4}}\left(\mathcal{S}_{\infty}^{+} ; \mathcal{S}_{\infty}^{-}\right)$.

Let $\beta_{A^{(k)}}=\beta_{A^{(k)}}\left(\sigma_{x}^{(k, 1)}, \sigma_{x}^{(k, 2)}\right) \in \mathcal{B}_{A^{(k)}}\left(\mathcal{S}_{1} ; \mathcal{S}_{2}\right), k=1,2$.

Clearly $\beta_{A_{k}} \supseteq \beta_{A^{(k)}} \cup \beta_{A_{3}}^{(k)} \cup \beta_{A_{4}}^{(k)}, k=1,2$.

Let $\pi_{1}$ be a $\left(\beta_{1} \cup \beta_{A^{(1)}}\right)$-partition of $[c, d]$.

Let $\pi_{2}$ be a $\left(\beta_{1} \cup \beta_{A^{(2)}}\right)$-partition of $[c, d]$.

By Lemma 4.2 there exists $\pi_{3}$, a $\left(\beta_{3} \cup \beta_{A_{3}}\right)$-partition of $[a, c]$. Clearly $\pi_{3}$ is both, a $\left(\beta_{3} \cup \beta_{A_{3}}^{(1)}\right)$ partition and a $\left(\beta_{3} \cup \beta_{A_{3}}^{(2)}\right)$ partition of $[a, c]$.

By Lemma 4.2 there exists $\pi_{4}$, a $\left(\beta_{4} \cup \beta_{A_{4}}\right)$-partition of $[d, b]$. Clearly $\pi_{4}$ is both, a $\left(\beta_{4} \cup \beta_{A_{4}}^{(1)}\right)$-partition and a $\left(\beta_{4} \cup \beta_{A_{4}}^{(2)}\right)$ partition of $[d, b]$.

Let $\pi^{(k)}=\pi_{k} \cup \pi_{3} \cup \pi_{4}, k=1,2$. Then $\pi^{(1)}$ and $\pi^{(2)}$ are $\left(\beta \cup \beta_{A_{k}}\right)$-partitions of $[a, b]($ see $(4))$.

We have

$$
\left|s\left(f, \pi^{(1)}\right)-s\left(f, \pi^{(2)}\right)\right|<\epsilon \text {, hence }\left|s\left(f, \pi_{1}\right)-s\left(f, \pi_{2}\right)\right|<\epsilon .
$$

By Lemma 7.2 it follows that $f$ is $\left[\mathcal{S}_{1} \mathcal{S}_{2} \mathcal{R}\right]$-integrable on $[c, d]$.

Lemma 7.4 (A quasi Saks-Henstock lemma). Let $\mathcal{S}_{1}=\left\{\mathcal{S}_{1}(x)\right\}_{x \in \mathbb{R}}$ be a local system $\mathcal{S}_{\infty}^{+}$-filtering on $[a, b)$, and let $\mathcal{S}_{2}=\left\{\mathcal{S}_{2}(x)\right\}_{x \in \mathbb{R}}$ be a local system $\mathcal{S}_{\infty}^{-}$filtering on $(a, b]$. Let $f:[a, b] \rightarrow \mathbb{R}$ be $\left[\mathcal{S}_{1} \mathcal{S}_{2} \mathcal{R}\right]$-integrable on $[a, b]$. Let $F:[a, b] \rightarrow \mathbb{R}, F(a)=0, F(x)=\left[\mathcal{S}_{1} \mathcal{S}_{2} \mathcal{R}\right] \int_{a}^{x} f(t) d t, x \in(a, b]$. For every $\epsilon>0$ there exists $\beta=\beta\left(\left\{X_{i}\right\},\left\{\delta_{i}\right\}\right) \in \overline{\mathcal{B}}_{[a, b]}$, such that 
(i) $|s(f, \pi)-S(F, \pi)|<\epsilon$, whenever $\pi$ is a $\beta$-partial partition of $[a, b]$.

(ii) $\sum_{([x, y], t) \in \pi}|f(t)(y-x)-(F(y)-F(x))|<2 \epsilon$ whenever $\pi$ is a $\beta$-partial partition of $[a, b]$.

Proof. Let $\epsilon>0$. For $\frac{\epsilon}{2}$ and $F(b)$ let $\beta=\beta\left(\left\{X_{i}\right\},\left\{\delta_{i}\right\}\right)$ be given by Definition 7.1. Let $\pi$ be a $\beta$-partial partition of $[a, b]$.

(i) Let $\left(c_{k}, d_{k}\right), k=1,2, \ldots, n$ be the components of the open set $(a, b) \backslash$ $\left(\cup_{(I, t) \in \pi} I\right)$. By Lemma 7.3 we have $\left[\mathcal{S}_{1} \mathcal{S}_{2} \mathcal{R}\right] \int_{c_{k}}^{d_{k}} f(t) d t=F\left(d_{k}\right)-F\left(c_{k}\right)$. For each $k=1,2, \ldots, n$ let $\beta^{(k)}=\beta^{(k)}\left(\left\{Y_{j}^{(k)}\right\},\left\{\delta_{j}^{(k)}\right\}\right) \in \overline{\mathcal{B}}_{\left[c_{k}, d_{k}\right]}$ be given by Definition 7.1. Let $A^{(k)}=\operatorname{Is}\left(\left\{Y_{j}^{(k)}\right\}\right), k=1,2, \ldots n$ and let $A_{o}=\cup_{k=1}^{n} A^{(k)} \cup$ Is $\left(\left\{X_{i}\right\}\right)$. By Definition 7.1 it follows that for $\epsilon /(2 n), A^{(k)}$ and $\beta^{(k)}$ there exists $\beta_{A^{(k)}}=\beta_{A^{(k)}}\left(\sigma_{x}^{(k, 1)}, \sigma_{x}^{(k, 2)}\right) \in \mathcal{B}_{A^{(k)}}\left(\mathcal{S}_{1} ; \mathcal{S}_{2}\right)$ such that $\mid s\left(f, \pi^{\prime}\right)-\left(F\left(d_{k}\right)-\right.$ $\left.F\left(c_{k}\right)\right) \mid<\epsilon /(2 n)$ whenever $\pi^{\prime}$ is a $\beta_{k}$ partition of $\left[c_{k}, d_{k}\right]$, where $\beta_{k}=\beta^{(k)} \cup$ $\beta_{A^{(k)}}$. For $\epsilon / 2, A_{o}$ and $\beta$ there exists $\beta_{A_{o}}=\beta_{A_{o}}\left(\sigma_{x}^{(0,1)}, \sigma_{x}^{(0,2)}\right) \in \mathcal{B}_{A_{o}}\left(\mathcal{S}_{1} ; \mathcal{S}_{2}\right)$ such that $\left|s\left(f, \pi^{\prime}\right)-F(b)\right|<\epsilon / 2$ whenever $\pi^{\prime}$ is a $\beta_{o}$-partition of $[a, b]$, where $\beta_{o}=\beta \cup \beta_{A_{o}}$. Let

$$
\begin{gathered}
\sigma_{x}^{(k,+)}=\sigma_{x}^{(0,1)} \cap \sigma_{x}^{(k, 1)} \in \mathcal{S}_{\infty}^{+}(x), \text { if } x \in A^{(k)} \cap\left[c_{k}, d_{k}\right) ; \\
\sigma_{x}^{(k,-)}=\sigma_{x}^{(0,2)} \cap \sigma_{x}^{(k, 2)} \in \mathcal{S}_{\infty}^{-}(x), \text { if } x \in A^{(k)} \cap\left(c_{k}, d_{k}\right] .
\end{gathered}
$$

Clearly $\left\{X_{i} \cap Y_{j}^{(k)}\right\}_{(i, j)} \in \overline{\mathcal{P}}_{\left[c_{k}, d_{k}\right]}$. Let

$$
\begin{aligned}
& \delta_{i, j}^{(k)}: X_{i} \cap Y_{j}^{(k)} \rightarrow(0,+\infty), \delta_{i, j}(x)<\min \left\{\delta_{i}(x), \delta_{j}^{(k)}(x)\right\} ; \\
& \alpha_{k}=\alpha_{k}\left(\left\{X_{i} \cap Y_{j}^{(k)}\right\}, \delta_{i, j}^{(k)}, \sigma_{x}^{(k,+)}, \sigma_{x}^{(k,-)}\right) \in \mathcal{A}\left(\overline{\mathcal{P}}_{\left[c_{k}, d_{k}\right]} ; \mathcal{S}_{\infty}^{+} ; \mathcal{S}_{\infty}^{-}\right) .
\end{aligned}
$$

Let $\pi^{(k)}$ be an $\alpha_{k}$-partition of $\left[c_{k}, d_{k}\right], k=1,2, \ldots, n$ (see Lemma 4.2). Then $\pi^{(k)}$ is also a $\beta_{k^{-}}$partition and a $\beta_{o}$-partition of $\left[c_{k}, d_{k}\right]$. Then $\pi_{o}=\pi \cup$ $\left(\cup_{k=1}^{n} \pi^{(k)}\right)$ is a $\beta_{o^{-}}$partition of $[a, b]$. Since $F(b)=S\left(F, \pi_{o}\right)$, it follows that $\left|s\left(f, \pi_{o}\right)-S\left(F, \pi_{o}\right)\right|<\epsilon / 2$. We have $\mid s(f, \pi)-S(F, \pi)+\sum_{k=1}^{n}\left(F\left(d_{k}\right)-F\left(c_{k}\right)-\right.$ $\left.s\left(f, \pi^{(k)}\right)\right) \mid<\epsilon / 2$, hence $|s(f, \pi)-S(F, \pi)|<\epsilon$.

(ii) Let $\pi_{1}=\{([x, y], t) \in \pi: f(t)(y-x)>F(y)-F(x)\}$ and $\pi_{2}=$ $\{([x, y], t) \in \pi: f(t)(y-x) \leq F(y)-F(x)\}$. Then $\pi=\pi_{1} \cup \pi_{2}$. By (i) we have $\left|s\left(f, \pi_{1}\right)-S\left(F, \pi_{1}\right)\right|<\epsilon$ and $\left|s\left(f, \pi_{2}\right)-S\left(F, \pi_{2}\right)\right|<\epsilon$. Thus $\sum_{([x, y], t) \in \pi} \mid f(t)(y-$ $x)-(F(y)-F(x)) \mid<\epsilon+\epsilon=2 \epsilon$.

Corollary 7.1. Let $\mathcal{S}_{1}=\left\{\mathcal{S}_{1}(x)\right\}_{x \in \mathbb{R}}$ be a local system $\mathcal{S}_{\infty}^{+}$-filtering on $[a, b)$, and let $\mathcal{S}_{2}=\left\{\mathcal{S}_{2}(x)\right\}_{x \in \mathbb{R}}$ be a local system $\mathcal{S}_{\infty}^{-}$-filtering on $(a, b]$. Let $f$ : $[a, b] \rightarrow \mathbb{R}$ be $\left[\mathcal{S}_{1} \mathcal{S}_{2} \mathcal{R}\right]$-integrable on $[a, b]$. Let $F:[a, b] \rightarrow \mathbb{R}, F(a)=0$, 
$F(x)=\left[\mathcal{S}_{1} \mathcal{S}_{2} \mathcal{R}\right] \int_{a}^{x} f(t) d t, x \in(a, b]$. Then $F \in N_{\mathcal{B}_{o}}$ on $[a, b]$, hence $F \in(N)$ on $[a, b]$.

Proof. Let $\epsilon>0$ and $Z \subset[a, b],|Z|=0$. By Lemma 5.4 .5 of [1], there exists $\delta: Z \rightarrow(0,+\infty)$ such that $|s(f ; \pi)|<\epsilon$, whenever $\pi$ is a McShane $\delta$-fine partial partition (i.e., if $([x, y], t) \in \pi$ then $[x, y] \subset(t-\delta(t), t+\delta(t)))$ of $[a, b]$, with all tags in $Z$. By Lemma 7.4, (i), there exists $\beta=\beta\left(\left\{X_{i}\right\}_{i},\left\{\delta_{i}\right\}_{i}\right) \in \overline{\mathcal{B}}_{[a, b]}$, such that $|s(f, \pi)-S(F, \pi)|<\epsilon$, whenever $\pi$ is a $\beta$-partial partition of $[a, b]$. Let $Z_{i}=Z \cap X_{i}$ and let $\delta_{i}^{\star}: Z_{i} \rightarrow(0,+\infty), \delta_{i}^{\star}(x)=\min \left\{\delta(x), \delta_{i}(x)\right\}$. Let $\beta_{o}=\beta_{o}\left(\left\{Z_{i}\right\},\left\{\delta_{i}^{\star}\right\}\right) \in \mathcal{B}_{Z}$ and let $\pi_{o}$ be a $\beta_{o}$-partial partition of $Z$. Then $\pi_{o}$ is also a $\beta$-partial partition of $[a, b]$. It follows that $\left|S\left(F, \pi_{o}\right)\right| \leq \mid s\left(f, \pi_{o}\right)-$ $S\left(F, \pi_{o}\right)|+| s\left(f, \pi_{o}\right) \mid<2 \epsilon$ (see Lemma 7.4).

Lemma 7.5. Let $\mathcal{S}_{1}=\left\{\mathcal{S}_{1}(x)\right\}_{x \in \mathbb{R}}$ be a local system $\mathcal{S}_{\infty}^{+}$-filtering on $[a, b)$, and let $\mathcal{S}_{2}=\left\{\mathcal{S}_{2}(x)\right\}_{x \in \mathbb{R}}$ be a local system $\mathcal{S}_{\infty}^{-}$-filtering on $(a, b]$. Let $f$ : $[a, b] \rightarrow \mathbb{R}$ be $\left[\mathcal{S}_{1} \mathcal{S}_{2} \mathcal{R}\right]$-integrable on $[a, b]$. Let $F:[a, b] \rightarrow \mathbb{R}, F(a)=0$, $F(x)=\left[\mathcal{S S}_{2} \mathcal{R}\right] \int_{a}^{x} f(t) d t, x \in(a, b]$. Then $F_{a p}^{\prime}(x)=f(x)$ a.e. on $[a, b]$.

Proof. Let $A=\{x \in(a, b)$ : there exists $\alpha(x)>0$ with the following property: for every $\eta(x)>0$, with $(x-\eta(x), x+\eta(x)) \subset(a, b)$, and for every $D_{x}$ with $\underline{d}^{i}\left(D_{x}, x\right)=1$, there exists $y \in D_{x} \cap(x-\eta(x), x+\eta(x))$ such that $\mid F(y)-$ $F(x)-f(x)(y-x) \mid>\alpha(x)(y-x)\}$. Let $A_{n}=\{x \in(a, b): \alpha(x) \geq 1 / n\}$. Then $A=\cup_{n=1}^{\infty} A_{n}$. By Lemma 7.4 (ii), for $\epsilon>0$, there exists $\beta=\beta\left(\left\{X_{i}\right\},\left\{\delta_{i}\right\}\right) \in$ $\overline{\mathcal{B}}_{[a, b]}$ such that $\sum_{([x, y], t) \in \pi}|F(y)-F(x)-f(t)(y-x)|<\epsilon$, whenever $\pi$ is a $\beta$-partial partition of $[a, b]$. Let $A_{n, i}=\left\{x \in A_{n} \cap X_{i}: d\left(X_{i}, x\right)<1\right\}$. By the Lebesgue Density Theorem (see for example [1, p. 10]) it follows that $\left|A_{n, i}\right|=0$. Let $B_{n}=A_{n} \backslash\left(\cup_{i=1}^{\infty} A_{n, i}\right)$. Clearly $\left|A_{n}\right|=\left|B_{n}\right|$. If $x \in B_{n}$ and $x \in X_{i}$ for some $i$ then $d\left(X_{i}, x\right)=1$ (indeed, if $d\left(X_{i}, x\right) \neq 1$ then $x \in A_{n, i}$, a contradiction). For $\delta_{i}(x)>\eta(x)>0$ with $(x-\eta(x), x+\eta(x)) \subset(a, b)$, there exists $y \in X_{i} \cap(x-\eta(x), x+\eta(x))$ such that $|F(y)-F(x)-f(x)(y-x)| \geq$ $(1 / n)|y-x|$. If $y>x$ then $([x, y], x) \in \beta$ and if $y<x$ then $([y, x], x) \in \beta$. Let $\mathcal{A}=\left\{[x, y]: z \in B_{n} \cap\{x, y\}\right.$ and $\left.([x, y], z) \in \beta\right\}$. Then $\mathcal{A}$ is a Vitali cover of $B_{n}$, hence by Vitali's Covering Theorem (see for example [1, p. 11]) there exists $\pi$, a $\beta$-partial partition of $[a, b]$ such that $\left|B_{n}\right| \leq \epsilon+\sum_{([x, y], z) \in \pi}(y-x) \leq$ $\epsilon+n \cdot \sum_{([x, y], z) \in \pi}|F(y)-F(x)-f(z)(y-x)|<\epsilon+n \epsilon$. Since $\epsilon$ is arbitrary, it follows that $\left|B_{n}\right|=0$, hence $|A|=0$. Therefore $F_{a p}^{\prime}(x)=f(x)$ on $(a, b) \backslash A$. Thus $F_{a p}^{\prime}=f$ a.e. on $[a, b]$.

Lemma 7.6. Let $f:[a, b] \rightarrow \mathbb{R}, f \in\left[\mathcal{S}_{1} \mathcal{S}_{2} \mathcal{R}\right]$-integrable on $[a, b]$, and let $F(x)=\left[\mathcal{S}_{1} \mathcal{S}_{2} \mathcal{R}\right] \int_{a}^{x} f(t) d t, x \in[a, b]$. Then $F \in V B G$ on $[a, b]$. 
Proof. For $\epsilon=1$ let $\beta=\beta\left(\left\{X_{i}\right\},\left\{\delta_{i}\right\}\right) \in \overline{\mathcal{B}}_{[a, b]}$ be given by Definition 7.1. Clearly $\cup_{i=1}^{\infty} X_{i}=[a, b]$. Let

$$
\begin{aligned}
& X_{i j}=\left\{x \in X_{i}: \delta_{i}(x)>(b-a) / j\right\}, j=1,2, \ldots ; \\
& X_{i j k}=X_{i j} \cap\left[a+(k-1) \frac{b-a}{j}, a+k \frac{b-a}{j}\right], k=1,2, \ldots, j ; \\
& X_{i j k m}=\left\{x \in X_{i j k}:|f(x)|<m\right\}, m=1,2, \ldots
\end{aligned}
$$

Clearly $[a, b]=\cup_{i, j, k, m} X_{i j k m}$. Let $\left\{\left[a_{n}, b_{n}\right]\right\}, n=1,2, \ldots, p$ be a finite set of nonoverlapping intervals with endpoints in $X_{i j k m}$. Then $\left(\left[a_{n}, b_{n}\right], a_{n}\right) \in \beta$. By Lemma 7.4, $\sum_{n=1}^{p}\left|f\left(a_{n}\right)\left(b_{n}-a_{n}\right)-\left(F\left(b_{n}\right)-F\left(a_{n}\right)\right)\right|<2$. It follows that $\sum_{n=1}^{p}\left|F\left(b_{n}\right)-F\left(a_{n}\right)\right| \leq \sum_{n=1}^{p}\left|f\left(a_{n}\right)\left(b_{n}-a_{n}\right)-\left(F\left(b_{n}\right)-F\left(a_{n}\right)\right)\right|+$ $\sum_{n=1}^{p}\left|f\left(a_{n}\right)\right|\left(b_{n}-a_{n}\right)<2+m(b-a)$, hence $F \in V B$ on $X_{i j k m}$. Therefore $F$ is also $V B G$ on $[a, b]$.

Remark 7.1. To prove Lemma 7.5 and Lemma 7.6 we have followed the technique used by Lee and Soedijono in Theorem 4.2 of [12], and our proof is based on a quasi Saks- Henstock type lemma, i.e., Lemma 7.4 (see Section 1). But we do not know if either of the two integrals, the $A H$ integral and the $\left[\mathcal{S}_{1} \mathcal{S}_{2} \mathcal{R}\right]$-integral, satisfy a Saks-Henstock type lemma.

\section{The Ward Type $\left[\mathcal{S}_{1} \mathcal{S}_{2} \mathcal{W}\right]$ Integral}

Definition 8.1. Let $\mathcal{S}_{1}=\left\{\mathcal{S}_{1}(x)\right\}_{x \in \mathbb{R}}$ be a local system $\mathcal{S}_{\infty}^{+}$-filtering on $[a, b)$, and let $\mathcal{S}_{2}=\left\{\mathcal{S}_{2}(x)\right\}_{x \in \mathbb{R}}$ be a local system $\mathcal{S}_{\infty}^{-}$-filtering on $(a, b]$. Let $f$ : $[a, b] \rightarrow \mathbb{R}$.

- We define the following classes of majorants:

$\left[\overline{\mathcal{S}_{1} \mathcal{S}_{2} \mathcal{W}}\right](f ;[a, b])=\left\{M:[a, b] \rightarrow \mathbb{R}: M(a)=0 ; M \in\left(\mathcal{S}_{1} ; \mathcal{S}_{2}\right) \mathcal{C}_{i}\right.$ on $[a, b]$; there exists a $\beta=\beta\left(\left\{X_{i}\right\},\left\{\delta_{i}\right\}\right) \in \overline{\mathcal{B}}_{[a, b]}$ such that $M(y)-M(x) \geq$ $f(t)(y-x)$, whenever $([x, y], t) \in \beta$.

- We define the following class of minorants:

$\left[\underline{\mathcal{S}_{1} \mathcal{S}_{2} \mathcal{W}}\right](f ;[a, b])=\left\{m:[a, b] \rightarrow \mathbb{R}:-m \in\left[\overline{\mathcal{S}_{1} \mathcal{S}_{(2)} \mathcal{W}}\right](-f ;[a, b])\right\}$

- If $\left[\overline{\mathcal{S}_{1} \mathcal{S}_{2} \mathcal{W}}\right](f ;[a, b]) \neq \emptyset$ then we denote by $\bar{J}_{f}(x)$ (or simply $\bar{J}(x)$ ), $x \in[a, b]$ the lower bound of all $M(x), M \in\left[\overline{\mathcal{S}_{1} \mathcal{S}_{2} \mathcal{W}}\right](f ;[a, b])$.

- If $\left[\mathcal{S}_{1} \mathcal{S}_{2} \mathcal{W}\right](f ;[a, b]) \neq \emptyset$ then we denote by $\underline{J}_{f}(x)$ (or simply $\underline{J}(x)$ ), $x \in[a, b]$ the upper bound of all $m(x), m \in\left[\underline{\mathcal{S}_{1} \mathcal{S}_{2} \mathcal{W}}\right](f ;[a, b])$. 
- If $\left[\overline{\mathcal{S}_{1} \mathcal{S}_{2} \mathcal{W}}\right](f ;[a, b]) \times\left[\underline{\mathcal{S}_{1} \mathcal{S}_{2} \mathcal{W}}\right](f ;[a, b]) \neq \emptyset$ and $\bar{J}(b)=\underline{J}(b)$ then $f$ is said to be $\left[\mathcal{S}_{1} \mathcal{S}_{2} \mathcal{W}\right]$-integrable on $[a, b]$. In this case we write $\bar{J}(b)=$ $\underline{J}(b)=\left[\mathcal{S}_{1} \mathcal{S}_{2} \mathcal{W}\right] \int_{a}^{b} f(t) d t$.

Lemma 8.1. Let $\mathcal{S}_{1}=\left\{\mathcal{S}_{1}(x)\right\}_{x \in \mathbb{R}}$ and $\mathcal{S}_{2}=\left\{\mathcal{S}_{2}(x)\right\}_{x \in \mathbb{R}}$ be local systems such that $\mathcal{S}_{1} \ll \mathcal{S}_{\infty}^{+}$on $[a, b)$ and $\mathcal{S}_{2} \ll \mathcal{S}_{\infty}^{-}$on $(a, b]$. Let $F, f:[a, b] \rightarrow \mathbb{R}$ and let $A=\left\{a_{1}, a_{2}, a-3, \ldots\right\}$ be a countable subset of $(a, b)$. If $F \in\left(\mathcal{S}_{1} ; \mathcal{S}_{2}\right) \mathcal{C}_{i}$ on $[a, b]$ then for $\epsilon>0$, there exist $\beta_{A}=\beta_{A}\left(\sigma_{x}^{(1)}, \sigma_{x}^{(2)}\right) \in \mathcal{B}_{A}\left(\mathcal{S}_{1} ; \mathcal{S}_{2}\right)$ and $H:[a, b] \rightarrow \mathbb{R}$ such that:

(i) $H(a)=0 ; H(b)<\epsilon$;

(ii) $H$ is increasing on $[a, b]$;

(iii) $(F+H)(x)-(F+H)(t) \geq f(t)(x-t)$ whenever $x \in[t, b) \cap \sigma_{t}^{(1)}, t \in\{a\} \cup A$ and $(F+H)(t)-(F+H)(x) \geq f(t)(t-x)$ whenever $x \in(a, t] \cap \sigma_{t}^{(2)}$, $t \in\{b\} \cup A$.

(iv) $F+H \in\left(\mathcal{S}_{1} ; \mathcal{S}_{2}\right) \mathcal{C}_{i}$ on $[a, b]$.

Moreover, if $F \in\left(\mathcal{S}_{1} ; \mathcal{S}_{2}\right) \mathcal{C}$ on $[a, b]$ then $|F(y)-F(x)-f(t)(y-x)| \leq H(y)-$ $H(x), \quad$ whenever $([x, y], t) \in \beta_{A}\left(\sigma_{x}^{1)}, \sigma_{x}^{(2)}\right)$.

Proof. Let $\epsilon>0$. Since $F \in\left(\mathcal{S}_{1} ; \mathcal{S}_{2}\right) \mathcal{C}_{i}$ on $[a, b]$ it follows that there exists $S_{a}^{(1)} \in \mathcal{S}_{1}(a)$ such that

$$
F(x)-F(a)>-\frac{\epsilon}{2^{3}} \quad \text { whenever } \quad x \in S_{a}^{(1)}, \quad x>a,
$$

and for every $a_{i}$ there exists $S_{a_{i}}^{(1)} \in \mathcal{S}_{1}\left(a_{i}\right)$ such that

$$
F(x)-F\left(a_{i}\right)>-\frac{\epsilon}{2^{i+3}} \quad \text { whenever } \quad x \in S_{a_{i}}^{(1)}, \quad x>a-i .
$$

Let $H_{1}:[a, b] \rightarrow \mathbb{R}, H_{1}(a)=0$,

$$
H_{1}(x)=\frac{\epsilon}{2^{2}}+\sum_{a_{i}<x} \frac{\epsilon}{2^{i+3}} .
$$

Clearly $H_{1}$ is increasing and $H_{1}(b)=\epsilon / 2$. Let $x>a$. Then $H_{1}(x)-H_{1}(a) \geq$ $\epsilon / 2^{2}$, so

$$
\left(F+H_{1}\right)(x)-\left(F+H_{1}\right)(a)>\frac{\epsilon}{2^{2}}-\frac{\epsilon}{2^{3}}=\frac{\epsilon}{2^{3}}>|f(a)|(x-a),
$$


whenever

$$
x \in S_{a}^{(1)} \cap\left(a-\frac{\epsilon}{(|f(a)|+1) \cdot 2^{3}}, a+\frac{\epsilon}{(|f(a)|+1) \cdot 2^{3}}\right)=: \sigma_{a}^{(1)} \text { and } x>a .
$$

Let $x>a_{j}$. Then $H_{1}(x)-H_{1}\left(a_{j}\right) \geq \epsilon / 2^{j+2}$, so

$$
\left(F+H_{1}\right)(x)-\left(F+H_{1}\right)\left(a_{j}\right)>\frac{\epsilon}{2^{j+2}}-\frac{\epsilon}{2^{j+3}}=\frac{\epsilon}{2^{j+3}}>\left|f\left(a_{j}\right)\right|\left(x-a_{j}\right),
$$

whenever

$$
x \in S_{a_{j}}^{(1)} \cap\left(a_{j}-\frac{\epsilon}{\left(\left|f\left(a_{j}\right)\right|+1\right) \cdot 2^{j+3}}, a_{j}+\frac{\epsilon}{\left(\left|f\left(a_{j}\right)\right|+1\right) \cdot 2^{j+3}}\right)=: \sigma_{a_{j}}^{(1)}
$$

and $x>a_{j}$. Similarly, there exists $S_{b}^{(2)} \in \mathcal{S}_{2}(b)$ such that

$$
F(y)-F(b)<\frac{\epsilon}{2^{3}} \quad \text { whenever } \quad y \in S_{b}^{(2)}, \quad y<b,
$$

and for every $a_{i}$ there exists $S_{a_{i}}^{(2)} \in \mathcal{S}_{2}\left(a_{i}\right)$ such that

$$
F(y)-F\left(a_{i}\right)<\frac{\epsilon}{2^{i+3}} \quad \text { whenever } \quad y \in S_{a_{i}}^{(2)}, \quad y<a_{i} .
$$

Let $H_{2}:[a, b] \rightarrow \mathbb{R}, H_{2}(a)=0$,

$$
H_{2}(x)=\sum_{a_{i} \leq x} \frac{\epsilon}{2^{i+2}} .
$$

Clearly $H_{2}$ is increasing and $H_{2}(b)=\epsilon / 2$. Let

$$
\sigma_{b}^{(2)}:=S_{b}^{(2)} \cap\left(b-\frac{\epsilon}{(|f(b)|+1) \cdot 2^{3}}, b+\frac{\epsilon}{(|f(b)|+1) \cdot 2^{3}}\right)
$$

and

$$
\sigma_{a_{i}}^{(2)}:=S_{a_{i}}^{(2)} \cap\left(a_{i}-\frac{\epsilon}{\left(\left|f\left(a_{i}\right)\right|+1\right) \cdot 2^{i+3}}, a_{i}+\frac{\epsilon}{\left(\left|f\left(a_{i}\right)\right|+1\right) \cdot 2^{i+3}}\right) .
$$

Then $\left(F+H_{2}\right)(t)-\left(F+H_{2}\right)(x) \geq f(t)(t-x)$ whenever $x \in(a, t] \cap \sigma_{t}^{(2)}$, $t \in\{b\} \cup A$.

The function $H=H_{1}+H_{2}$ satisfies the required properties.

If $F \in\left(\mathcal{S}_{1} ; \mathcal{S}_{2}\right) \mathcal{C}$ on $[a, b]$ then, for example, $S_{a_{i}}^{(1)}$ may be chosen such that

$$
\left|F(x)-F\left(a_{i}\right)\right|<\frac{\epsilon}{2^{3}} \quad \text { whenever } \quad x \in S_{a_{i}}^{(1)}, \quad x>a_{i} .
$$


Then for $x \in \sigma_{a_{i}}^{(1)}, x>a_{i}$ it follows that

$$
\begin{gathered}
\left|F(x)-F\left(a_{i}\right)-f\left(a_{i}\right)\left(x-a_{i}\right)\right|<\left|F(x)-F\left(a_{i}\right)\right|+\left|f\left(a_{i}\right)\right|\left(x-a_{i}\right)< \\
<\frac{\epsilon}{2^{3}}+\frac{\epsilon}{2^{3}}=\frac{\epsilon}{2^{2}} \leq H_{1}(x)-H_{1}\left(a_{i}\right) \leq H(x)-H\left(a_{i}\right) .
\end{gathered}
$$

Lemma 8.2. Let $\mathcal{S}_{1}=\left\{\mathcal{S}_{1}(x)\right\}_{x \in \mathbb{R}}$ be a local system $\mathcal{S}_{\infty}^{+}$-filtering on $[a, b)$, and let $\mathcal{S}_{2}=\left\{\mathcal{S}_{2}(x)\right\}_{x \in \mathbb{R}}$ be a local system $\mathcal{S}_{\infty}^{-}$-filtering on $(a, b]$. Let $f:[a, b] \rightarrow \mathbb{R}$. If $\left[\overline{\mathcal{S}_{1} \mathcal{S}_{2} \mathcal{W}}\right](f ;[a, b]) \times\left[\underline{\left.\mathcal{S}_{1} \mathcal{S}_{2} \mathcal{W}\right]}(f ;[a, b]) \neq \emptyset\right.$ and $(M, m) \in\left[\overline{\mathcal{S}_{1} \mathcal{S}_{2} \mathcal{W}}\right](f ;[a, b]) \times$ $\left[\mathcal{S}_{1} \mathcal{S}_{2} \mathcal{W}\right](f ;[a, b])$ then we have

(i) $M-m$ is positive and increasing on $[a, b]$, hence $M(b) \geq m(b)$;

(ii) $M-\underline{J}$ is positive and increasing on $[a, b]$, hence $M(b) \geq \underline{J}(b)$;

(iii) $\bar{J}-m$ is positive and increasing on $[a, b]$, hence $\bar{J}(b) \geq m(b)$;

(iv) $\bar{J}-\underline{J}$ is positive and increasing on $[a, b]$, hence $\bar{J}(b) \geq \underline{J}(b)$.

Proof. (i) Let $a \leq c<d \leq b$. For $M$ there exists a $\beta^{(1)}=\beta^{(1)}\left(\left\{X_{i}^{(1)}\right\},\left\{\delta_{i}^{(1)}\right\}\right)$ $\in \overline{\mathcal{B}}_{[a, b]}$ such that $M(y)-M(x) \geq f(t)(y-x)$, whenever $([x, y], t) \in \beta^{(1)}$. For $m$ there exists a $\beta^{(2)}=\beta^{(2)}\left(\left\{X_{i}^{(2)}\right\},\left\{\delta_{i}^{(2)}\right\}\right) \in \overline{\mathcal{B}}_{[a, b]}$ such that $m(y)-m(x) \leq$ $f(t)(y-x)$, whenever $([x, y], t) \in \beta^{(2)}$.

Let $A=\operatorname{Is}\left(\left\{X_{i}^{(1)} \cap X_{j}^{(2)} \cap[c, d]\right\}_{i, j}\right)$ and let $\epsilon>0$. By Lemma 8.1, there exist $\beta_{A}^{(k)}=\beta_{A}^{(k)}\left(\sigma_{x}^{(k, 1)}, \sigma_{x}^{(k, 2)}\right) \in \mathcal{B}_{A}\left(\mathcal{S}_{1} ; \mathcal{S}_{2}\right)$ and $H_{k}:[c, d] \rightarrow \mathbb{R}, k=1,2, H_{k}(c)=$ $0, H_{k}(d) \leq \epsilon, H_{k}$ increasing, such that $M(y)-M(x)+H_{1}(y)-H_{1}(x) \geq f(t)(y-$ $x)$, whenever $([x, y], t) \in \beta_{A}^{(1)}$ and $m(y)-m(x)-\left(H_{2}(y)-H_{2}(x)\right) \leq f(t)(y-x)$, whenever $([x, y], t) \in \beta_{A}^{(2)}$. Let

$$
\begin{aligned}
& \delta_{i, j}: X_{i}^{(1)} \cap X_{j}^{(2)} \cap[c, d] \rightarrow(0,+\infty), \delta_{i, j}(x)=\min \left\{\delta_{i}^{(1)}(x), \delta_{j}^{(2)}(x)\right\} . \\
& \beta=\beta\left(\left\{X_{i}^{(1)} \cap X_{j}^{(2)} \cap[c, d]\right\},\left\{\delta_{i, j}\right\}\right) \in \overline{\mathcal{B}}_{[c, d]} . \\
& \sigma_{x}^{(1)}=\sigma_{x}^{(1,1)} \cap \sigma_{x}^{(2,1)}, \text { for } x \in[c, d) \cap A . \\
& \sigma_{x}^{(2)}=\sigma_{x}^{(1,2)} \cap \sigma_{x}^{(2,2)}, \text { for } x \in(c, d] \cap A \\
& \beta_{A}=\beta_{A}\left(\sigma_{x}^{(1)}, \sigma_{x}^{(2)}\right) \in \mathcal{B}_{A}\left(\mathcal{S}_{\infty}^{+}, \mathcal{S}_{\infty}^{-}\right) .
\end{aligned}
$$


By Lemma 4.2 there exists $\pi$, a $\beta \cup \beta_{A}$-partition of $[c, d]$. If $([x, y], t) \in \beta \cup \beta_{A}$ then $([x, y], t) \in \beta^{(k)} \cup \beta_{A}^{(k)}, k=1,2$. It follows that $(M-m)(d)-(M-$ $m)(c)+2 \epsilon \geq\left(H_{1}+M\right)(d)-\left(H_{1}+M\right)(c)-\left(\left(m-H_{2}\right)(d)-\left(m-H_{2}\right)(c)\right)=$ $\sum_{([x, y], t) \in \pi}\left(\left(H_{1}+M\right)(y)-\left(H_{1}+M\right)(x)\right)-\sum_{([x, y], t) \in \pi}\left(\left(m-H_{2}\right)(y)-(m-\right.$ $\left.\left.H_{2}\right)(x)\right) \geq \sum_{([x, y], t) \in \pi}(f(t)(y-x)-f(t)(y-x))=0$. If $\epsilon \rightarrow 0$ then we obtain that $M-m$ is increasing on $[a, b]$. Since $M(a)=m(a)=0, M-m$ is positive on $[a, b]$. Clearly $M(b) \geq m(b)$.

(ii) By (i) we have $M(d)-M(c) \geq m(d)-m(c) \geq m(d)-\underline{J}(c)$, hence $M(d)-M(c) \geq \underline{J}(d)-\underline{J}(c)$ whenever $a \leq c<d \leq b$. It follows that $M(d)-$ $\underline{J}(d) \geq M(c)-\underline{J}(c)$. Thus $M-\underline{J}$ is increasing and positive (since $M(a)=$ $\underline{J}(a)=0)$ on $[a, b]$. Clearly $M(b) \geq \underline{J}(b)$.

(iii) We have $M(d)-\bar{J}(c) \geq M(d)-M(c) \geq m(d)-m(c)$ (see (i)), hence $\bar{J}(d)-\bar{J}(c) \geq m(d)-m(c)$ whenever $a \leq c<d \leq b$. Therefore $\bar{J}-m$ is increasing and positive (since $\bar{J}(a)=m(a)=0$ ). Clearly $\bar{J}(b) \geq m(b)$.

(iv) We have $M(d)-\bar{J}(c) \geq M(d)-M(c) \geq \underline{J}(d)-\underline{J}(c)$ (see (ii)), hence $\bar{J}(d)-\bar{J}(c) \geq \underline{J}(d)-\underline{J}(c)$ whenever $a \leq c<\bar{d} \leq b$. Therefore $\bar{J}-\underline{J}$ is increasing and positive (since $\bar{J}(a)=\underline{J}(a)=0)$. Clearly $\bar{J}(b) \geq \underline{J}(b)$.

Lemma 8.3. Let $\mathcal{S}_{1}=\left\{\mathcal{S}_{1}(x)\right\}_{x \in \mathbb{R}}$ be a local system $\mathcal{S}_{\infty}^{+}$-filtering on $[a, b)$, and let $\mathcal{S}_{2}=\left\{\mathcal{S}_{2}(x)\right\}_{x \in \mathbb{R}}$ be a local system $\mathcal{S}_{\infty}^{-}$-filtering on $(a, b]$. Let $f:[a, b] \rightarrow \mathbb{R}$. The following assertions are equivalent:

(i) $f$ is $\left[\mathcal{S}_{1} \mathcal{S}_{2} \mathcal{W}\right]$ integrable on $[a, b]$.

(ii) $\left[\overline{\mathcal{S}_{1} \mathcal{S}_{2} \mathcal{W}}\right](f ;[a, b]) \times\left[\underline{\mathcal{S}_{1} \mathcal{S}_{2} \mathcal{W}}\right](f ;[a, b]) \neq \emptyset$ and for every $\epsilon>0$ there exists $(M, m) \in\left[\overline{\mathcal{S}_{1} \mathcal{S}_{2} \mathcal{W}}\right](\overline{f ;[a, b])}] \times\left[\underline{\mathcal{S}_{1} \mathcal{S}_{2} \mathcal{W}}\right](f ;[a, b])$ such that $M(b)-m(b)<$ $\epsilon$.

Proof. (i) $\Rightarrow$ (ii) For $\epsilon>0$ there exists a pair $(M, m) \in\left[\overline{\mathcal{S}_{1} \mathcal{S}_{2} \mathcal{W}}\right](f ;[a, b]) \times$ $\left[\mathcal{S}_{1} \mathcal{S}_{2} \mathcal{W}\right](f ;[a, b])$ such that $M(b)-\epsilon / 2<\bar{J}(b)=\underline{J}(b)<m(b)+\epsilon / 2$ (see the definitions of $\bar{J}(b)$ and $J(b))$, hence $M(b)-m(b)<\epsilon$.

(ii) $\Rightarrow$ (i) By Lemma 8.2, (iv) we have $0 \leq \bar{J}(b)-\underline{J}(b) \leq M(b)-m(b)<\epsilon$. Since $\epsilon$ is arbitrary, it follows that $\bar{J}(b)=\underline{J}(b)$.

Lemma 8.4. Let $\mathcal{S}_{1}=\left\{\mathcal{S}_{1}(x)\right\}_{x \in \mathbb{R}}$ be a local system $\mathcal{S}_{\infty}^{+}$-filtering on $[a, b)$, and let $\mathcal{S}_{2}=\left\{\mathcal{S}_{2}(x)\right\}_{x \in \mathbb{R}}$ be a local system $\mathcal{S}_{\infty}^{-}$-filtering on $(a, b]$. Let $f:[a, b] \rightarrow \mathbb{R}$ and $c \in(a, b)$. If $f$ is $\left[\mathcal{S}_{1} \mathcal{S}_{2} \mathcal{W}\right]$ integrable on $[a, b]$ then $f$ is $\left[\mathcal{S}_{1} \mathcal{S}_{2} \mathcal{W}\right]$ integrable on $[a, c]$ and on $[c, b]$, and

$$
\left[\mathcal{S}_{1} \mathcal{S}_{2} \mathcal{W}\right] \int_{a}^{b} f(t) d t=\left[\mathcal{S}_{1} \mathcal{S}_{2} \mathcal{W}\right] \int_{a}^{c} f(t) d t+\left[\mathcal{S}_{1} \mathcal{S}_{2} \mathcal{W}\right] \int_{c}^{b} f(t) d t
$$


Proof. By Lemma 8.3 it follows that $\left[\overline{\mathcal{S}_{1} \mathcal{S}_{2} \mathcal{W}}\right](f ;[a, b]) \times\left[\underline{\mathcal{S}_{1} \mathcal{S}_{2} \mathcal{W}}\right](f ;[a, b]) \neq$ $\emptyset$ and for every $\epsilon>0$ there exists $(M, m) \in\left[\overline{\mathcal{S}_{1} \mathcal{S}_{2} \mathcal{W}}\right](f ;[a, b]) \times\left[\underline{\mathcal{S}_{1} \mathcal{S}_{2} \mathcal{W}}\right](f ;[a, b])$ such that $M(b)-m(b)<\epsilon$.

Let $M_{1}=M_{/[a, c]}$ and $m_{1}=m_{/[a, c]}$. Let $M_{2}=M_{/[c, b]}-M(c)$ and $m_{2}=$ $m_{/[c, b]}-m(c)$. Then

$$
\left(M_{1}, m_{1}\right) \in\left[\overline{\mathcal{S}_{1} \mathcal{S}_{2} \mathcal{W}}\right](f ;[a, c]) \times\left[\underline{\mathcal{S}_{1} \mathcal{S}_{2} \mathcal{W}}\right](f ;[a, c]) \neq \emptyset .
$$

and

$$
\left(M_{2}, m_{2}\right) \in\left[\overline{\mathcal{S}_{1} \mathcal{S}_{2} \mathcal{W}}\right](f ;[c, b]) \times\left[\underline{\mathcal{S}_{1} \mathcal{S}_{2} \mathcal{W}}\right](f ;[c, b]) \neq \emptyset .
$$

We prove for example (6) ((7) follows similarly). Clearly $M \in\left(\mathcal{S}_{1} ; \mathcal{S}_{2}\right) \mathcal{C}_{i}$ on $[a, c]$. For $M$ there exists $\beta=\beta\left(\left\{X_{i}\right\},\left\{\delta_{i}\right\}\right) \in \overline{\mathcal{B}}_{[a, b]}$ such that $M(y)-M(x) \geq$ $f(t)(y-x)$, whenever $([x, y], t) \in \beta$. Then $\beta_{o}=\beta_{o}\left(\left\{X_{i} \cap[a, c]\right\},\left\{\left(\delta_{i}\right)_{/ X_{i} \cap[a, c]}\right\}\right)$ $\in \mathcal{B}_{[a, c]}$ and $\beta_{o} \subset \beta$. Then $M_{1}(y)-M_{1}(x) \geq f(t)(y-x)$, whenever $([x, y], t) \in$ $\beta_{o}$. Therefore $M_{1} \in\left[\overline{\mathcal{S}_{1} \mathcal{S}_{2} \mathcal{W}}\right](f ;[a, c])$. Similarly, $m_{1} \in\left[\underline{\mathcal{S}_{1} \mathcal{S}_{2} \mathcal{W}}\right](f ;[a, c])$. Thus we obtain that (6) is true.

By Lemma 8.2, (i) we have that $M_{1}(c)-m_{1}(c)<\epsilon$ and $M_{2}(c)-m_{2}(c)<\epsilon$. Therefore, by Lemma 8.3 , it follows that $f$ is $\left[\mathcal{S}_{1} \mathcal{S}_{2} \mathcal{W}\right]$-integrable on $[a, c]$ and $[c, b]$.

We also have $m_{1}(c)=m(c)<\left[\mathcal{S}_{1} \mathcal{S}_{2} \mathcal{W}\right] \int_{a}^{c} f(t) d t<M(c)=M_{1}(c)$ and $m_{2}(b)=m(b)-m(c) \leq\left[\mathcal{S}_{1} \mathcal{S}_{2} \mathcal{W}\right] \int_{c}^{b} f(t) d t \leq M(b)-M(c)=M_{2}(b)$. It follows that $m(b) \leq\left[\mathcal{S}_{1} \mathcal{S}_{2} \mathcal{W}\right] \int_{a}^{c} f(t) d t+\left[\mathcal{S}_{1} \mathcal{S}_{2} \mathcal{W}\right] \int_{c}^{b} f(t) d t \leq M(b)$. But $m(b) \leq\left[\mathcal{S}_{1} \mathcal{S}_{2} \mathcal{W}\right] \int_{c}^{b} f(t) d t \leq M(b)$ and $M(b)-m(b)<\epsilon$. Since $\epsilon$ is arbitrary we obtain (5).

Lemma 8.5. Let $\mathcal{S}_{1}=\left\{\mathcal{S}_{1}(x)\right\}_{x \in \mathbb{R}}$ be a local system, $\mathcal{S}_{\infty}^{+}$-filtering on $[a, b)$, and let $\mathcal{S}_{2}=\left\{\mathcal{S}_{2}(x)\right\}_{x \in \mathbb{R}}$ be a local system, $\mathcal{S}_{\infty}^{-}$-filtering on $(a, b]$. Let $f$ : $[a, b] \rightarrow \mathbb{R}$ and $c \in(a, b)$. If $f$ is $\left[\mathcal{S}_{1} \mathcal{S}_{2} \mathcal{W}\right]$-integrable on $[a, c]$ and on $[c, b]$, then $f$ is $\left[\mathcal{S}_{1} \mathcal{S}_{2} \mathcal{W}\right]$-integrable on $[a, b]$ and

$$
\left[\mathcal{S}_{1} \mathcal{S}_{2} \mathcal{W}\right] \int_{a}^{b} f(t) d t=\left[\mathcal{S}_{1} \mathcal{S}_{2} \mathcal{W}\right] \int_{a}^{c} f(t) d t+\left[\mathcal{S}_{1} \mathcal{S}_{2} \mathcal{W}\right] \int_{c}^{b} f(t) d t
$$

Proof. For $\epsilon>0$, let $\left(M_{1}, m_{1}\right) \in\left[\overline{\mathcal{S}_{1} \mathcal{S}_{2} \mathcal{W}}\right](f ;[a, c]) \times\left[\underline{\mathcal{S}_{1} \mathcal{S}_{2} \mathcal{W}}\right](f ;[a, c]) \neq \emptyset$ with $M_{1}(c)-m_{1}(c)<\epsilon$, and $\left(M_{2}, m_{2}\right) \in\left[\overline{\mathcal{S}_{1} \mathcal{S}_{2} \mathcal{W}}\right](f ;[c, b]) \times\left[\underline{\mathcal{S}_{1} \mathcal{S}_{2} \mathcal{W}}\right](f ;[c, b])$ $\neq \emptyset$ with $M_{2}(c)-m_{2}(c)<\epsilon$ (see Lemma 8.3). Let

$$
M(x)= \begin{cases}M_{1}(x) & , \quad x \in[a, c] \\ M_{1}(c)+M_{2}(x) & , x \in[c, b]\end{cases}
$$


and

$$
m(x)= \begin{cases}m_{1}(x) & , \quad x \in[a, c] \\ m_{1}(c)+m_{2}(x) & , \quad x \in[c, b] .\end{cases}
$$

For $M_{1}$ let $\beta_{1}=\beta_{1}\left(\left\{X_{i}^{(1)}\right\},\left\{\delta_{i}^{(1)}\right\}\right) \in \overline{\mathcal{B}}_{[a, c]}$ be given by Definition 8.1, and for $M_{2}$ let $\beta_{2}=\beta_{2}\left(\left\{X_{i}^{(2)}\right\},\left\{\delta_{i}^{(2)}\right\}\right) \in \overline{\mathcal{B}}_{[c, b]}$ be given by the same definition. Then $\left\{X_{i}^{(1)}\right\}_{i} \cup\left\{X_{i}^{(2)}\right\}_{i} \in \overline{\mathcal{P}}_{[a, b]}$. Let $\beta=\beta_{1} \cup \beta_{2}$. If $([x, y], t) \in \beta$ then either $([x, y], t) \in \beta_{1}$ or $([x, y], t) \in \beta_{2}$. In both cases we have $M(y)-$ $M(x) \geq f(t)(y-x)$. By Lemma 2.2 , (iii), $M \in\left(\mathcal{S}_{1} ; \mathcal{S}_{2}\right) \mathcal{C}_{i}$ on $[a, b]$. Therefore $M \in\left[\overline{\mathcal{S}}_{1} \mathcal{S}_{2} \mathcal{W}\right](f ;[a, b])$. Similarly we can show that $m \in\left[\mathcal{S}_{1} \mathcal{S}_{2} \mathcal{W}\right](f ;[a, b])$. Since $M(b)-m(b)<2 \epsilon$, by Lemma 8.3 , it follows that $\left.\overline{f \in\left[\mathcal{S}_{1}\right.} \mathcal{S}_{2} \mathcal{W}\right]$ on $[a, b]$. We also have $m_{1}(c)=m(c) \leq\left[\mathcal{S}_{1} \mathcal{S}_{2} \mathcal{W}\right] \int_{a}^{c} f(t) d t \leq M(c)=M_{1}(c)$ and $m_{2}(b)=m(b)-m(c) \leq\left[\mathcal{S}_{1} \mathcal{S}_{2} \mathcal{W}\right] \int_{c}^{b} f(t) d t \leq M_{2}(b)=M(b)-M(c)$. It follows that $m(b) \leq\left[\mathcal{S}_{1} \mathcal{S}_{2} \mathcal{W}\right] \int_{a}^{c} f(t) d t+\left[\mathcal{S}_{1} \mathcal{S}_{2} \mathcal{W}\right] \int_{c}^{b} f(t) d t \leq M(b)$. But $m(b) \leq\left[\mathcal{S}_{1} \mathcal{S}_{2} \mathcal{W}\right] \int_{a}^{b} f(t) d t \leq M(b)$ and $M(b)-m(b)<2 \epsilon$. Since $\epsilon$ is arbitrary we obtain (8).

Lemma 8.6. Let $f_{1}, f_{2}:[a, b] \rightarrow \mathbb{R}$ be $\left[\mathcal{S}_{1} \mathcal{S}_{2} \mathcal{W}\right]$-integrable on $[a, b]$, and let $\alpha_{1}, \alpha_{2} \in \mathbb{R}$. If $\mathcal{S}_{1}$ is filtering on $[a, b)$ and $\mathcal{S}_{2}$ is filtering on $(a, b]$, then $\alpha_{1} f_{1}+$ $\alpha_{2} f_{2}$ is $\left[\mathcal{S}_{1} \mathcal{S}_{2} \mathcal{W}\right]$ - integrable on $[a, b]$ and $\left[\mathcal{S}_{1} \mathcal{S}_{2} \mathcal{W}\right] \int_{a}^{b}\left(\alpha_{1} f_{1}+\alpha_{2} f_{2}\right)(t) d t=$ $\alpha_{1} \cdot\left[\mathcal{S}_{1} \mathcal{S}_{2} \mathcal{W}\right] \int_{a}^{b} f_{1}(t) d t+\alpha_{2} \cdot\left[\mathcal{S}_{1} \mathcal{S}_{2} \mathcal{W}\right] \int_{a}^{b} f_{2}(t) d t$

Proof. Let $(M, m) \in\left[\overline{\mathcal{S}_{1} \mathcal{S}_{2} \mathcal{W}}\right]\left(f_{1} ;[a, b]\right) \times\left[\underline{\mathcal{S}_{1} \mathcal{S}_{2} \mathcal{W}}\right]\left(f_{1} ;[a, b]\right) \neq \emptyset$. If $\alpha>0$ then $(\alpha M, \alpha m) \in\left[\overline{\mathcal{S}_{1} \mathcal{S}_{2} \mathcal{W}}\right]\left(\alpha f_{1} ;[a, b]\right) \times\left[\underline{\mathcal{S}_{1} \mathcal{S}_{2} \mathcal{W}}\right]\left(\alpha f_{1} ;[a, b]\right) \neq \emptyset$. Hence

$\alpha f_{1}$ is $\left[\mathcal{S}_{1} \mathcal{S}_{2} \mathcal{W}\right]$ - integrable and

$$
\left[\mathcal{S}_{1} \mathcal{S}_{2} \mathcal{W}\right] \int_{a}^{b} \alpha f_{1}(t) d t=\alpha \cdot\left[\mathcal{S}_{1} \mathcal{S}_{2} \mathcal{W}\right] \int_{a}^{b} f_{1}(t) d t
$$

If $\alpha<0$ then $(\alpha m, \alpha M) \in\left[\overline{\mathcal{S}_{1} \mathcal{S}_{2} \mathcal{W}}\right]\left(\alpha f_{1} ;[a, b]\right) \times\left[\underline{\mathcal{S}_{1} \mathcal{S}_{2} \mathcal{W}}\right]\left(\alpha f_{1} ;[a, b]\right) \neq \emptyset$ and (9) is valid.

It remains to prove that the lemma is true for $\alpha_{1}=\beta_{1}=1$.

Let $M_{k} \in\left[\overline{\mathcal{S}_{1} \mathcal{S}_{2} \mathcal{W}}\right]\left(f_{k} ;[a, b]\right) \neq \emptyset, k=1,2$

For $M_{k}$ let $\beta^{(k)}=\beta^{(k)}\left(\left\{X_{i}^{(k)}\right\},\left\{\delta_{i}^{(k)}\right\}\right) \in \overline{\mathcal{B}}_{[a, b]}$ be given by Definition 8.1, $k=1,2$.

Let $X_{i j}=X_{i}^{(1)} \cap X_{j}^{(2)}$. Then $\left\{X_{i j}\right\}_{i, j} \in \overline{\mathcal{P}}_{[a, b]}$. 
Let $\delta_{i j}: X_{i j} \rightarrow(0,+\infty), \delta_{i j}(x)=\min \left\{\delta_{i}^{(1)}(x), \delta_{j}^{(2)}(x)\right\}$.

Let $\beta=\beta\left(\left\{X_{i j}\right\},\left\{\delta_{i j}\right\}\right) \in \overline{\mathcal{B}}_{[a, b]}$.

Let $([x, y], t) \in \beta$. Clearly $([x, y], t)$ also belongs to $\beta^{(k)}, k=1,2$, so $M_{k}(y)-$ $M_{k}(x) \geq f_{k}(t)(y-x)$.

It follows that $\left(M_{1}+M_{2}\right)(y)-\left(M_{1}+M_{2}\right)(x) \geq\left(f_{1}(t)+f_{2}(t)\right)(y-x)$. Since $\mathcal{S}_{1}$ is filtering on $[a, b)$ and $\mathcal{S}_{2}$ is filtering on $(a, b]$, it follows that $M_{1}+M_{2} \in$ $\left(\mathcal{S}_{1} ; \mathcal{S}_{2}\right) \mathcal{C}_{i}$ on $[a, b]$. Hence $M_{1}+M_{2} \in\left[\overline{\mathcal{S}_{1} \mathcal{S}_{2} \mathcal{W}}\right]\left(f_{1}+f_{2} ;[a, b]\right)$ and $\bar{J}_{f_{1}+f_{2}}(b) \leq$ $\bar{J}_{f_{1}}(b)+\bar{J}_{f_{2}}(b)$. Similarly we obtain that $\underline{J}_{f_{1}}(b)+\underline{J}_{f_{2}}(b) \leq \underline{J}_{f_{1}+f_{2}}(b)$. By Lemma 8.2, (iv) we obtain that $f_{1}+f_{2} \in\left[\mathcal{S}_{1} \mathcal{S}_{2} \mathcal{W}\right]$ and $\left[\mathcal{S}_{1} \mathcal{S}_{2} \mathcal{W}\right] \int_{a}^{b}\left(f_{1}+\right.$ $\left.f_{2}\right)(t) d t=\left[\mathcal{S}_{1} \mathcal{S}_{2} \mathcal{W}\right] \int_{a}^{b} f_{1}(t) d t+\left[\mathcal{S}_{1} \mathcal{S}_{2} \mathcal{W}\right] \int_{a}^{b} f_{2}(t) d t$

\section{The Variational Type $\left[\mathcal{S}_{1} \mathcal{S}_{2} \mathcal{V}\right]$-Integral}

Definition 9.1. Let $\mathcal{S}_{1}=\left\{\mathcal{S}_{1}(x)\right\}_{x \in \mathbb{R}}$ be a local system $\mathcal{S}_{\infty}^{+}$-filtering on $[a, b)$, and let $\mathcal{S}_{2}=\left\{\mathcal{S}_{2}(x)\right\}_{x \in \mathbb{R}}$ be a local system $\mathcal{S}_{\infty}^{-}$-filtering on $(a, b]$. Let $f$ : $[a, b] \rightarrow \mathbb{R} . \quad f$ is said to be $\left[\mathcal{S}_{1} \mathcal{S}_{2} \mathcal{V}\right]$-integrable on $[a, b]$, if there exists $H$ : $[a, b] \rightarrow \mathbb{R}, H \in\left(\mathcal{S}_{1} ; \mathcal{S}_{2}\right) \mathcal{C}$, with the following property: for every $\epsilon>0$ there exist $\beta=\beta\left(\left\{X_{i}\right\},\left\{\delta_{i}\right\}\right) \in \overline{\mathcal{B}}_{[a, b]}$ and $G:[a, b] \rightarrow \mathbb{R}$, such that $G(a)=0, G(b) \leq$ $\epsilon, G$ is increasing on $[a, b]$, and $|H(y)-H(x)-f(t)(y-x)|<G(y)-G(x)$, whenever $([x, y], t) \in \beta$. $H$ is called the $\left[\mathcal{S}_{1} \mathcal{S}_{2} \mathcal{V}\right]$ indefinite integral of $f$ on $[a, b]$ and $\left[\mathcal{S}_{1} \mathcal{S}_{2} \mathcal{V}\right] \int_{a}^{b} f(t) d t=H(b)-H(a)$.

Lemma 9.1. Let $\mathcal{S}_{1}=\left\{\mathcal{S}_{1}(x)\right\}_{x \in \mathbb{R}}$ be a local system $\mathcal{S}_{\infty}^{+}$-filtering on $[a, b)$, and let $\mathcal{S}_{2}=\left\{\mathcal{S}_{2}(x)\right\}_{x \in \mathbb{R}}$ be a local system $\mathcal{S}_{\infty}^{-}$-filtering on $(a, b]$. Let $f:[a, b] \rightarrow$ $\mathbb{R}$. If $H_{1}, H_{2}:[a, b] \rightarrow \mathbb{R}$ are $\left[\mathcal{S}_{1} \mathcal{S}_{2} \mathcal{V}\right]$ indefinite integrals of $f$ on $[a, b]$ then $H_{1}(b)-H_{1}(a)=H_{2}(b)-H_{2}(a)$. Therefore the $\left[\mathcal{S}_{1} \mathcal{S}_{2} \mathcal{V}\right]$ integral is well defined.

Proof. Let $H_{k}:[a, b] \rightarrow \mathbb{R}, H_{k} \in\left(\mathcal{S}_{1} ; \mathcal{S}_{2}\right) \mathcal{C}, k=1,2$, satisfying the following property: for $\epsilon>0$ there exist $\beta^{(k)}=\beta^{(k)}\left(\left\{X_{i}^{(k)}\right\},\left\{\delta_{i}^{(k)}\right\}\right) \in \overline{\mathcal{B}}_{[a, b]}$ and $G^{(k)}:[a, b] \rightarrow \mathbb{R}$ such that $G^{(k)}(a)=0, G^{(k)}(b) \leq \epsilon, G^{(k)}$ is increasing on $[a, b]$ and $\left|H^{(k)}(y)-H^{(k)}(x)-f(t)(y-x)\right|<G^{(\bar{k})}(y)-G^{(k)}(x)$, whenever $([x, y], t) \in \beta^{(k)}, k=1,2$. Let $X_{m, n}=X_{m}^{(1)} \cap X_{n}^{(2)}$. Then $\left\{X_{m, n}\right\}_{m, n} \in \overline{\mathcal{P}}_{[a, b]}$. Let $A=\operatorname{Is}\left(\left\{X_{m, n}\right\}\right)$. By Lemma 8.1, there exist $\beta_{A}^{(k)}=\beta_{A}^{(k)}\left(\sigma_{x}^{(k, 1)}, \sigma_{x}^{(k, 2)}\right) \in$ $\mathcal{B}_{A}\left(\mathcal{S}_{1} ; \mathcal{S}_{2}\right)$ and $h^{(k)}:[a, b] \rightarrow \mathbb{R}, h^{(k)}(a)=0, h^{(k)}(b) \leq \epsilon, h^{(k)}$ increasing, such that $\left|H^{(k)}(y)-H^{(k)}(x)-f(t)(y-x)\right| \leq h^{(k)}(y)-h^{(k)}(x)$, whenever $([x, y], t) \in \beta^{(k)}, k=1,2$. Let $\sigma_{x}^{+}=\sigma_{x}^{(1,1)} \cap \sigma_{x}^{(2,1)}$ and $\sigma_{x}^{-}=\sigma_{x}^{(1,2)} \cap \sigma_{x}^{(2,2)}$. Let $\delta_{m, n}: X_{m, n} \rightarrow(0,+\infty), \delta_{m, n}(x)=\min \left\{\delta_{m}^{(1)}(x), \delta_{n}^{(2)}(x)\right\}$. Let $\beta=$ 
$\beta\left(\left\{X_{m, n}\right\},\left\{\delta_{m, n}\right\}\right) \in \overline{\mathcal{B}}_{[a, b]}$ and $\beta_{A}=\beta_{A}\left(\sigma_{x}^{+}, \sigma_{x}^{-}\right) \in \mathcal{B}_{A}\left(\mathcal{S}_{\infty}^{+}, \mathcal{S}_{\infty}^{-}\right)$. Then, by Lemma 4.2 there exists $\pi$, a $\beta \cup \beta_{A}$-partition of $[a, b]$. But $\pi$ is also a $\beta^{(1)} \cup \beta_{A}^{(1)}$ - and a $\left(\beta^{(2)} \cup \beta_{A}^{(2)}\right)$-partition of $[a, b]$. Therefore $\mid\left(H_{1}-H_{2}\right)(b)-\left(H_{1}-\right.$ $\left.H_{2}\right)(a)|=| \sum_{([x, y], t) \in \pi}\left(H_{1}-H_{2}\right)(y)-\left(H_{1}-H_{2}\right)(x)|=| \sum_{([x, y], t) \in \pi}\left(H_{1}(y)-\right.$ $H_{1}(x)-f(t)(y-x)-\left(H_{2}(y)-H_{2}(x)-f(t)(y-x)\right)\left|\leq \sum_{([x, y], t) \in \pi}\right| H_{1}(y)-$ $H_{1}(x)-f(t)(y-x)\left|+\sum_{([x, y], t) \in \pi}\right| H_{2}(y)-H_{2}(x)-f(t)(y-x) \mid \leq \sum_{([x, y], t) \in \pi \cap \beta}$ $\left|G^{(1)}(y)-G^{(1)}(x)\right|+\sum_{([x, y], t) \in \pi \cap \beta}\left|G^{(2)}(y)-G^{2)}(x)\right|+\sum_{([x, y], t) \in \pi \cap \beta_{A}^{(1)}} \mid H_{1}(y)-$ $H_{1}(x)-f(t)(y-x)\left|+\sum_{([x, y], t) \in \pi \cap \beta_{A}^{(2)}}\right| H_{2}(y)-H_{2}(x)-f(t)(y-x) \mid \leq G^{(1)}(b)+$ $G^{(2)}(b)+h^{(1)}(b)+h^{(2)}(b) \leq 4 \epsilon$. Since $\epsilon$ is arbitrary, it follows that $H_{1}(b)-$ $H_{1}(a)=H_{2}(b)-H_{2}(a)$.

Lemma 9.2. Let $\mathcal{S}_{1}=\left\{\mathcal{S}_{1}(x)\right\}_{x \in \mathbb{R}}$ be a local system, $\mathcal{S}_{\infty}^{+}$-filtering on $[a, b)$, and let $\mathcal{S}_{2}=\left\{\mathcal{S}_{2}(x)\right\}_{x \in \mathbb{R}}$ be a local system, $\mathcal{S}_{\infty}^{-}$-filtering on $(a, b]$. Let $f$ : $[a, b] \rightarrow \mathbb{R}$ and $a<c<b$. If $f$ is $\left[\mathcal{S}_{1} \mathcal{S}_{2} \mathcal{V}\right]$-integrable on $[a, b]$ then $f$ is also $\left[\mathcal{S}_{1} \mathcal{S}_{2} \mathcal{V}\right]$-integrable on $[a, c]$ and on $[c, b]$, and

$$
\left[\mathcal{S}_{1} \mathcal{S}_{2} \mathcal{V}\right] \int_{a}^{c} f(t) d t+\left[\mathcal{S}_{1} \mathcal{S}_{2} \mathcal{V}\right] \int_{c}^{b} f(t) d t=\left[\mathcal{S}_{1} \mathcal{S}_{2} \mathcal{V}\right] \int_{a}^{b} f(t) d t
$$

Proof. Since $f$ is $\left[\mathcal{S}_{1} \mathcal{S}_{2} \mathcal{V}\right]$ integrable on $[a, b]$, there exists a function $H$ : $[a, b] \rightarrow \mathbb{R}, H \in\left(\mathcal{S}_{1} ; \mathcal{S}_{2}\right) \mathcal{C}$ satisfying the following property: for every $\epsilon>0$, there exist $\beta=\beta\left(\left\{X_{i}\right\},\left\{\delta_{i}\right\}\right) \in \overline{\mathcal{B}}_{[a, b]}$ and $G:[a, b] \rightarrow \mathbb{R}$, such that $G(a)=0$, $G(b) \leq \epsilon, G$ is increasing on $[a, b]$, and $|H(y)-H(x)-f(t)(y-x)|<G(y)-$ $G(x)$, whenever $([x, y], t) \in \beta$.

We define

$$
\begin{aligned}
& X_{i}^{(1)}=X_{i} \cap[a, c] \text { and } X_{i}^{(2)}=X_{i} \cap[c, b] \text {; Clearly }\left\{X_{i}^{(1)}\right\}_{i} \in \overline{\mathcal{P}}_{[a, c]} \text { and }\left\{X_{i}^{(2)}\right\}_{i} \in \\
& \overline{\mathcal{P}}_{[c, b]} \text {; } \\
& \beta^{(1)}=\beta^{(1)}\left(\left\{X_{i}^{(1)}\right\},\left\{\delta_{i}\right\}\right) \in \overline{\mathcal{B}}_{[a, c]} ; \\
& \beta^{(2)}=\beta^{(2)}\left(\left\{X_{i}^{(2)}\right\},\left\{\delta_{i}\right\}\right) \in \overline{\mathcal{B}}_{[c, b]} ; \\
& H_{1}, G_{1}:[a, c] \rightarrow \mathbb{R}, H_{1}(x)=H(x), G_{1}(x)=G(x) . \\
& H_{2}, G_{2}:[c, b] \rightarrow \mathbb{R}, H_{2}(x)=H(x), G_{2}(x)=G(x)-G(c) \text {. }
\end{aligned}
$$

By Lemma 2.2 , (iii), $H_{1}, H_{2} \in\left(\mathcal{S}_{1} ; \mathcal{S}_{2}\right) \mathcal{C}$ on $[a, c]$ respectively $[c, b]$. If $([x, y], t)$ $\in \beta^{(1)}$ then $([x, y], t) \in \beta$, hence $f$ is $\left[\mathcal{S}_{1} \mathcal{S}_{2} \mathcal{V}\right]$-integrable on $[a, c]$, and we have $\left[\mathcal{S}_{1} \mathcal{S}_{2} \mathcal{V}\right] \int_{a}^{c} f(t) d t=H(c)-H(a)$. Similarly it follows that $f$ is $\left[\mathcal{S}_{1} \mathcal{S}_{2} \mathcal{V}\right]$ integrable on $[c, b]$, and $\left[\mathcal{S}_{1} \mathcal{S}_{2} \mathcal{V}\right] \int_{c}^{b} f(t) d t=H(b)-H(c)$.

Since $\left[\mathcal{S}_{1} \mathcal{S}_{2} \mathcal{V}\right] \int_{a}^{b} f(t) d t=H(b)-H(a)$, we obtain (10). 
Lemma 9.3. Let $\mathcal{S}_{1}=\left\{\mathcal{S}_{1}(x)\right\}_{x \in \mathbb{R}}$ be a local system, $\mathcal{S}_{\infty}^{+}$-filtering on $[a, b)$, and let $\mathcal{S}_{2}=\left\{\mathcal{S}_{2}(x)\right\}_{x \in \mathbb{R}}$ be a local system, $\mathcal{S}_{\infty}^{-}$-filtering on $(a, b]$. Let $f$ : $[a, b] \rightarrow \mathbb{R}$ and $a<c<b$. If $f$ is $\left[\mathcal{S}_{1} \mathcal{S}_{2} \mathcal{V}\right]$-integrable on $[a, c]$ and $[c, b]$ then $f$ is also $\left[\mathcal{S}_{1} \mathcal{S}_{2} \mathcal{V}\right]$-integrable on $[a, b]$ and

$$
\left[\mathcal{S}_{1} \mathcal{S}_{2} \mathcal{V}\right] \int_{a}^{c} f(t) d t+\left[\mathcal{S}_{1} \mathcal{S}_{2} \mathcal{V}\right] \int_{c}^{b} f(t) d t=\left[\mathcal{S}_{1} \mathcal{S}_{2} \mathcal{V}\right] \int_{a}^{b} f(t) d t
$$

Proof. Let $I_{1}=[a, c]$ and $I_{2}=[c, b]$. Let $H_{k}: I_{k} \rightarrow \mathbb{R}, H_{k} \in\left(\mathcal{S}_{1} ; \mathcal{S}_{2}\right) \mathcal{C}$, such that for every $\epsilon>0$, there exist $\beta_{k}=\beta_{k}\left(\left\{X_{i}^{(k)}\right\},\left\{\delta_{i}^{(k)}\right\}\right) \in \overline{\mathcal{B}}_{I_{k}}, k=1,2$, and $G_{k}: I_{k} \rightarrow(0,+\infty)$ such that $G_{k}$ is increasing, $G_{1}(a)=0, G_{1}(c)<\epsilon$, $G_{2}(c)=0, G_{2}(b)<\epsilon$, and $\left|f(t)(y-x)-\left(H_{k}(y)-H_{k}(x)\right)\right|<G_{k}(y)-G_{k}(x)$, whenever $([x, y], t) \in \beta_{k}, k=1,2$.

Let $H:[a, b] \rightarrow \mathbb{R}$,

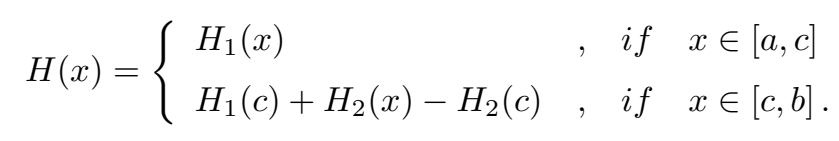

Let $G:[a, b] \rightarrow(0,+\infty)$,

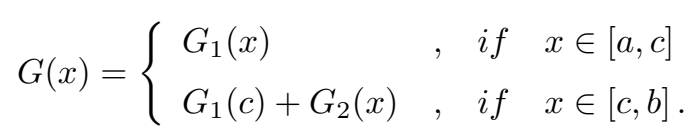

Let $\beta=\beta_{1} \cup \beta_{2}$. If $([x, y], t) \in \beta$ then either $([x, y], t) \in \beta_{1}$ or $([x, y], t) \in \beta_{2}$, and clearly in both cases we have $|f(t)(y-x)-(H(y)-H(x))|<G(y)-G(x)$. Therefore $f$ is $\left[\mathcal{S}_{1} \mathcal{S}_{2} \mathcal{V}\right]$-integrable on $[a, b]$ and $\left[\mathcal{S}_{1} \mathcal{S}_{2} \mathcal{V}\right] \int_{a}^{b} f(t) d t=H(b)-$ $H(a)=H(b)-H(c)+H(c)-H(a)=H_{2}(b)-H_{2}(c)+H_{1}(c)-H_{1}(a)=$ $\left[\mathcal{S}_{1} \mathcal{S}_{2} \mathcal{V}\right] \int_{c}^{b} f(t) d t+\left[\mathcal{S}_{1} \mathcal{S}_{2} \mathcal{V}\right] \int_{a}^{c} f(t) d t$

Lemma 9.4. Let $f_{1}, f_{2}:[a, b] \rightarrow \mathbb{R}$ be $\left[\mathcal{S}_{1} \mathcal{S}_{2} \mathcal{V}\right]$-integrable on $[a, b]$, and let $\alpha_{1}, \alpha_{2} \in \mathbb{R}$. If $\mathcal{S}_{1}$ is filtering on $[a, b)$ and $\mathcal{S}_{2}$ is filtering on $(a, b]$, then $\alpha_{1} f_{1}+$ $\alpha_{2} f_{2}$ is $\left[\mathcal{S}_{1} \mathcal{S}_{2} \mathcal{V}\right]$ - integrable on $[a, b]$ and $\left[\mathcal{S}_{1} \mathcal{S}_{2} \mathcal{V}\right] \int_{a}^{b}\left(\alpha_{1} f_{1}+\alpha_{2} f_{2}\right)(t) d t=\alpha_{1}$. $\left[\mathcal{S}_{1} \mathcal{S}_{2} \mathcal{V}\right] \int_{a}^{b} f_{1}(t) d t+\alpha_{2} \cdot\left[\mathcal{S}_{1} \mathcal{S}_{2} \mathcal{V}\right] \int_{a}^{b} f_{2}(t) d t$

Proof. Let $\alpha_{k} \neq 0$.

For $f_{k}$ let $H_{k}:[a, b] \rightarrow \mathbb{R}, k=1,2, H_{k} \in\left(\mathcal{S}_{1} ; \mathcal{S}_{2}\right) \mathcal{C}$, with the following property: for every $\epsilon>0$ there exist $\beta^{(k)}=\beta^{(k)}\left(\left\{X_{i}^{(k)}\right\},\left\{\delta_{i}^{(k)}\right\}\right) \in \overline{\mathcal{B}}_{[a, b]}$, and $G_{k}:[a, b] \rightarrow \mathbb{R}$, such that $G_{1}(a)=0, G_{1}(b) \leq \frac{\epsilon}{2\left|\alpha_{2}\right|}, G_{2}(a)=0$, $G_{2}(b)<\frac{\epsilon}{2\left|\alpha_{1}\right|}, G_{k}$ is increasing on $[a, b]$, and $\mid H_{k}(y)-H_{k}(x)-f(t)(y-$ $x) \mid<G_{k}(y)-G_{k}(x)$, whenever $([x, y], t) \in \beta^{(k)}$. 
Let $X_{i j}=X_{i}^{(1)} \cap X_{j}^{(2)}$. Then $\left\{X_{i j}\right\}_{i, j} \in \overline{\mathcal{P}}_{[a, b]}$.

Let $\delta_{i j}: X_{i j} \rightarrow(0,+\infty), \delta_{i j}(x)=\min \left\{\delta_{i}^{(1)}(x), \delta_{j}^{(2)}(x)\right\}$.

Let $\beta=\beta\left(\left\{X_{i j}\right\},\left\{\delta_{i j}\right\}\right) \in \overline{\mathcal{B}}_{[a, b]}$.

Let $\left.G(x)=\left|\alpha_{1}\right| G_{1}(x)+\left|\alpha_{2}\right| G_{2} x\right)$. Clearly $G(a)=0, G(b)<\epsilon$ and $G$ is increasing on $[a, b]$.

Let $([x, y], t) \in \beta$. Clearly $([x, y], t)$ also belongs to $\beta^{(k)}, k=1,2$.

Then we have $\mid\left(\alpha_{1} H_{1}+\alpha_{2} H_{2}\right)(y)-\left(\alpha_{1} H_{1}+\alpha_{2} H_{2}\right)(x)-\left(\alpha_{1} f_{1}+\alpha_{2} f_{2}\right)(t)(y-$ $x)|\leq| \alpha_{1}|\cdot| H_{1}(y)-H_{1}(x)-f_{1}(t)(y-x)|+| \alpha_{2}|\cdot| H_{2}(y)-H_{2}(x)-f_{2}(t)(y-x) \mid<$ $\left|\alpha_{1}\right|\left(G_{1}(y)-G_{1}(x)\right)+\left|\alpha_{2}\right|\left(G_{2}(y)-G_{2}(x)\right)=G(y)-G(x)$. But $\alpha_{1} H_{1}+$ $\alpha_{2} H_{2} \in\left(\mathcal{S}_{1} ; \mathcal{S}_{2}\right) \mathcal{C}$ on $[a, b]$. It follows that $\alpha_{1} H_{1}+\alpha_{2} H_{2}$ is the $\left[\mathcal{S}_{1} \mathcal{S}_{2} \mathcal{V}\right]$ indefinite integral of $\alpha_{1} f_{1}+\alpha_{2} f_{2}$ on $[a, b]$ and $\left[\mathcal{S}_{1} \mathcal{S}_{2} \mathcal{V}\right] \int_{a}^{b}\left(\alpha_{1} f_{1}+\alpha_{2} f_{2}\right)(t) d t=$ $\alpha_{1} \cdot\left[\mathcal{S}_{1} \mathcal{S}_{2} \mathcal{V}\right] \int_{a}^{b} f_{1}(t) d t+\alpha_{2} \cdot\left[\mathcal{S}_{1} \mathcal{S}_{2} \mathcal{V}\right] \int_{a}^{b} f_{2}(t) d t$

\section{The Relations Between the $\left[\mathcal{S}_{1} \mathcal{S}_{2} \mathcal{R}\right]-$, the $\left[\mathcal{S}_{1} \mathcal{S}_{2} \mathcal{W}\right]$ - and the $\left[\mathcal{S}_{1} \mathcal{S}_{2} \mathcal{V}\right]$-Integrals}

Definition 10.1. Let $\mathcal{S}_{1}=\left\{\mathcal{S}_{1}(x)\right\}_{x \in \mathbb{R}}$ be a local system, $\mathcal{S}_{\infty}^{+}$-filtering on $[a, b)$, and let $\mathcal{S}_{2}=\left\{\mathcal{S}_{2}(x)\right\}_{x \in \mathbb{R}}$ be a local system, $\mathcal{S}_{\infty}^{-}$-filtering on $(a, b]$. A function $f:[a, b] \rightarrow \mathbb{R}$ is said to be $b i\left[\mathcal{S}_{1} \mathcal{S}_{2} \mathcal{V}\right]$-integrable on $[a, b]$ if there exists $H:[a, b] \rightarrow \mathbb{R}, H \in\left(\mathcal{S}_{1} ; \mathcal{S}_{2}\right) \mathcal{C}_{i} \cap\left(\mathcal{S}_{1} ; \mathcal{S}_{2}\right) \mathcal{C}_{d}$, satisfying the following property: for every $\epsilon>0$ there exists $\beta^{(k)}=\beta^{(k)}\left(\left\{X_{i}^{(k)}\right\},\left\{\delta_{i}^{(k)}\right\}\right) \in \overline{\mathcal{B}}_{[a, b]}, k=1,2$, and $G_{k}:[a, b] \rightarrow[0,+\infty)$, with $G_{k}(a)=0, G_{k}(b) \leq \epsilon, G_{k}$ increasing on $[a, b]$, $k=1,2$ such that $H(y)-H(x)-f(t)(y-x)<G_{1}(y)-G_{1}(x)$, whenever $([x, y], t) \in \beta^{(1)}$ and $-H(y)+H(x)+f(t)(y-x)<G_{2}(y)-G_{2}(x)$ whenever $([x, y], t) \in \beta^{(2)} . H$ is called the $b i\left[\mathcal{S}_{1} \mathcal{S}_{2} \mathcal{V}\right]$-indefinite integral of $f$ on $[a, b]$ and we write $b i\left[\mathcal{S}_{1} \mathcal{S}_{2} \mathcal{V}\right] \int_{a}^{b} f(t) d t=H(b)-H(a)$.

Lemma 10.1. Let $\mathcal{S}_{1}=\left\{\mathcal{S}_{1}(x)\right\}_{x \in \mathbb{R}}$ be a local system, $\mathcal{S}_{\infty}^{+}$-filtering on $[a, b)$, and let $\mathcal{S}_{2}=\left\{\mathcal{S}_{2}(x)\right\}_{x \in \mathbb{R}}$ be a local system, $\mathcal{S}_{\infty}^{-}$-filtering on $(a, b]$. Let $f$ : $[a, b] \rightarrow \mathbb{R}$. Then we have:

(i) If $f$ is $\left[\mathcal{S}_{1} \mathcal{S}_{2} \mathcal{V}\right]$ - integrable on $[a, b]$ then $f$ is also bi $\left[\mathcal{S}_{1} \mathcal{S}_{2} \mathcal{V}\right]$-integrable on $[a, b]$, and the two integrals are equal. Moreover, if $\mathcal{S}_{1}$ is filtering on $[a, b)$ and $\mathcal{S}_{2}$ is filtering on $(a, b]$ then the $\left[\mathcal{S}_{1} \mathcal{S}_{2} \mathcal{V}\right]$-integral and the bi $\left[\mathcal{S}_{1} \mathcal{S}_{2} \mathcal{V}\right]$-integral are equivalent.

(ii) The following assertions are equivalent: 
a) $f$ is bi $\left[\mathcal{S}_{1} \mathcal{S}_{2} \mathcal{V}\right]$-integrable on $[a, b]$;

b) $f$ is $\left[\mathcal{S}_{1} \mathcal{S}_{2} \mathcal{W}\right]$-integrable on $[a, b]$,

and the two integrals are equal.

(iii) If $f$ is $\left[\mathcal{S}_{1} \mathcal{S}_{2} \mathcal{V}\right]$-integrable on $[a, b]$ then $f$ is also $\left[\mathcal{S}_{1} \mathcal{S}_{2} \mathcal{W}\right]$-integrable on $[a, b]$ and the two integrals are equal.

Proof. (i) The first part is obvious. We show the second part. For $\epsilon>0$ let $\beta_{k}=\beta_{k}\left(\left\{X_{i}^{(k)}\right\},\left\{\delta_{i}^{(k)}\right\}\right) \in \overline{\mathcal{B}}_{[a, b]}$ be given by Definition 10.1.

Let $X_{i j}=X_{i}^{(1)} \cap X_{j}^{(2)}$; then $\left\{X_{i j}\right\}_{i, j} \in \overline{\mathcal{P}}_{[a, b]}$.

Let $\delta_{i j}: X_{i j} \rightarrow(0,+\infty), \delta_{i j}(x)=\min \left\{\delta_{i}^{(1)}(x), \delta_{j}^{(2)}(x)\right\}$.

Let $\beta=\beta\left(\left\{X_{i j}\right\},\left\{\delta_{i j}\right\}\right) \in \overline{\mathcal{B}}_{[a, b]}$.

Let $A$ be a countable subset of $[a, b]$ that contains $\operatorname{Is}\left(\left\{X_{i j}\right\}\right)$; then $\operatorname{Is}\left(\left\{X_{i j}\right\}\right)$ $\supseteq \operatorname{Is}\left(\left\{X_{i}^{(1)}\right\}\right) \cup \operatorname{Is}\left(\left\{X_{j}^{(2)}\right\}\right)$.

Let $\beta_{A}^{(k)}=\beta_{A}^{(k)}\left(\sigma_{x}^{(k, 1)}, \sigma_{x}^{(k, 2)}\right) \in \mathcal{B}_{A}\left(\mathcal{S}_{1} ; \mathcal{S}_{2}\right)$ and $G_{k}:[a, b] \rightarrow(0,+\infty)$ be given by Definition 10.1, $k=1,2$.

Let $\sigma_{x}^{(1)}=\sigma_{x}^{(1,1)} \cap \sigma_{x}^{(2,1)} \in \mathcal{S}_{1}(x)$.

Let $\sigma_{x}^{(2)}=\sigma_{x}^{(1,2)} \cap \sigma_{x}^{(2,2)} \in \mathcal{S}_{2}(x)$.

Let $\beta_{A}=\beta_{A}\left(\sigma_{x}^{(1)}, \sigma_{x}^{(2)}\right) \in \mathcal{B}_{A}\left(\mathcal{S}_{1} ; \mathcal{S}_{2}\right)$.

Let $([x, y], t) \in \beta \cup \beta_{A}$. Then $([x, y], t) \in \beta^{(k)} \cup \beta_{A}^{(k)}, k=1,2$. It follows that $H(y)-H(x)-f(t)(y-x)<G_{1}(y)-G_{1}(a)$ and $-H(y)+H(x)+f(t)(y-x)<$ $G_{2}(y)-G_{2}(a)$. Let $G=G_{1}+G_{2}$. Then $|H(y)-H(x)-f(t)(y-x)|<$ $G(y)-G(x)$.

(ii) a) $\Rightarrow$ b) Let $\epsilon>0$. By Definition 10.1, there exist $H:[a, b] \rightarrow \mathbb{R}$ and $\beta^{(k)}=\beta^{(k)}\left(\left\{X_{i}^{(k)}\right\},\left\{\delta_{i}^{(k)}\right\}\right) \in \overline{\mathcal{B}}_{[a, b]}$ with the following property: for every countable subset $A^{(k)}$ of $[a, b]$ that contains $\operatorname{Is}\left(\left\{X_{i}^{(k)}\right\}\right), k=1,2$ there exist $\beta_{A^{(k)}}=\beta_{A^{(k)}}\left(\sigma_{x}^{(k, 1)}, \sigma_{x}^{(k, 2)}\right) \in \mathcal{B}_{A^{(k)}}\left(\mathcal{S}_{1} ; \mathcal{S}_{2}\right), k=1,2$ and $G_{k}:[a, b] \rightarrow[0,+\infty)$, with $G_{k}(a)=0, G_{k}(b)<\epsilon, G_{k}$ increasing on $[a, b], k=1,2$, such that $H(y)-$ $H(x)-f(t)(y-x)<G_{1}(y)-G_{1}(x)$, whenever $([x, y], t) \in \beta^{(1)} \cup \beta_{A^{(1)}}$ and $-H(y)+H(x)+f(t)(y-x)<G_{2}(y)-G_{2}(x)$, whenever $([x, y], t) \in \beta^{(2)} \cup \beta_{A^{(2)}}$. Let $M=H+G_{2}$ and $m=H-G_{1}$. Then $(M, m) \in\left[\overline{\mathcal{S}_{1} \mathcal{S}_{2} \mathcal{W}}\right](f ;[a, b]) \times$ 
$\left[\underline{\mathcal{S}_{1} \mathcal{S}_{2} \mathcal{W}}\right](f ;[a, b])$. It follows that $\bar{J}(b) \leq H(b)$ and $\underline{J}(b) \geq H(b)$. By Lemma 8.2 , (iv), we obtain that $\bar{J}(b) \geq \underline{J}(b)$, hence $H(b)=\left[\mathcal{S}_{1} \mathcal{S}_{2} \mathcal{W}\right] \int_{a}^{b} f(t) d t$.

b) $\Rightarrow$ a) Since $f$ is $\left[\mathcal{S}_{1} \mathcal{S}_{2} \mathcal{W}\right]$ - integrable on $[a, b]$, we have $\bar{J}(b)=\underline{J}(b)$ (see Definition 8.1). By Lemma 8.2, (iv) it follows that $\bar{J}-\underline{J}=0$ on $[a, b]$. Let $H(x)=\bar{J}(x)=\underline{J}(x), x \in[a, b]$. Clearly $H(a)=0$. For $\epsilon>0$ let $(M, m) \in\left[\overline{\mathcal{S}_{1} \mathcal{S}_{2} \mathcal{W}}\right](f ;[a, b]) \times\left[\underline{\mathcal{S}_{1} \mathcal{S}_{2} \mathcal{W}}\right](f ;[a, b]) \neq \emptyset$ such that $H(b)-\epsilon / 2<$ $m(b)$ and $M(b)<H(b)+\epsilon / 2$. By Definition 8.1, there exists a $\beta^{(k)}=$ $\eta^{(k)}\left(\left\{X_{i}^{(k)}\right\},\left\{\delta_{i}^{(k)}\right\}\right) \in \overline{\mathcal{B}}_{[a, b]}, k=1,2$ with the following property: for every countable subset $A^{(k)}$ of $[a, b]$ that contains $\operatorname{Is}\left(\left\{X_{i}^{(k)}\right\}\right), k=1,2$, there is a $\beta_{A^{(k)}}=\beta_{A^{(k)}}\left(\sigma_{x}^{(k, 1)}, \sigma_{x}^{(k, 2)}\right) \in \mathcal{B}_{A^{(k)}}\left(\mathcal{S}_{1} ; \mathcal{S}_{2}\right), k=1,2$, such that $M(y)-M(x) \geq$ $f(t)(y-x)$ whenever $([x, y], t) \in \beta^{(1)} \cup \beta_{A^{(1)}}$ and $m(y)-m(x) \leq f(t)(y-x)$ whenever $([x, y], t) \in \beta^{(2)} \cup \beta_{A^{(2)}}$. Let $G_{1}=H-m$ and $G_{2}=M-H$ on $[a, b]$. Then $H(y)-H(x)-f(t)(y-x) \leq G_{1}(y)-G_{1}(x)$ whenever $([x, y], t) \in$ $\beta^{(1)} \cup \beta_{A^{(1)}}$ and $f(t)(y-x)-(H(y)-H(x)) \leq G_{2}(y)-G_{2}(x)$, whenever $([x, y], t) \in \beta^{(2)} \cup \beta_{A^{(2)}}$.

(iii) See (i) and (ii).

Definition 10.2. Let $F:[a, b] \rightarrow \mathbb{R}$ and let $P$ be a closed subset of $[a, b]$, $c=\inf (P), d=\sup (P)$. Let $\left\{\left(c_{k}, d_{k}\right)\right\}_{k}$ be the intervals contiguous to $P$. We define the function $F_{P}:[c, d] \rightarrow \mathbb{R}$ such that $F_{P}(x)=F(x), x \in P$ and $F_{P}$ is linear on each $\left[c_{k}, d_{k}\right]$.

Lemma 10.2. Let $F:[a, b] \rightarrow \mathbb{R}$, let $P$ be a closed subset of $[a, b]$ and let $A$ be a measurable subset of $P$ such that $F \in V B$ on $P$. Then $F_{P}$ is derivable a.e. on $(\inf P, \sup P), F$ is approximately derivable a.e. on $A$ and $F_{P}^{\prime}(x)=F_{a p}^{\prime}(x)$ a.e. on $A$.

Proof. $F_{P}$ is $V B$ on $[\inf P, \sup P]$ (see for example $\left[1\right.$, p. 44]), hence $F_{P}$ is derivable a.e. on $(\inf P, \sup P)$. Let $P_{o}=\{x \in P: d(P, x)=1\}$. By Lebesgue's Density Theorem, $P_{o}$ is measurable and $\left|P_{o}\right|=|P|$. It follows that $F_{P}^{\prime}(x)=F_{a p}^{\prime}(x)$ a.e. on $P_{o}$, hence $F_{P}^{\prime}(x)=F_{a p}^{\prime}(x)$ a.e. on $A$.

Theorem 10.1. Let $\mathcal{S}_{1}=\left\{\mathcal{S}_{1}(x)\right\}_{x \in \mathbb{R}}$ be a local system, $\mathcal{S}_{\infty}^{+}$-filtering on $[a, b)$, and let $\mathcal{S}_{2}=\left\{\mathcal{S}_{2}(x)\right\}_{x \in \mathbb{R}}$ be a local system, $\mathcal{S}_{\infty}^{-}$-filtering on $(a, b]$. Let $f$ : $[a, b] \rightarrow \mathbb{R}$. If $f$ is $\left[\mathcal{S}_{1} \mathcal{S}_{2} \mathcal{D}\right]$ - integrable on $[a, b]$ then $f$ is also $\left[\mathcal{S}_{1} \mathcal{S}_{2} \mathcal{V}\right]$-integrable on $[a, b]$, and the two integrals are equal.

Proof. Since $f$ is $\left[\mathcal{S}_{1} \mathcal{S}_{2} \mathcal{D}\right]$-integrable on $[a, b]$, there exists a function $F$ : $[a, b] \rightarrow \mathbb{R}$ such that $F \in[A C G]$ and $F \in\left(\mathcal{S}_{1} ; \mathcal{S}_{2}\right) \mathcal{C}$ on $[a, b]$. Let $\left\{P_{i}\right\}_{i} \in \overline{\mathcal{P}}_{[a, b]}$ such that $F \in A C$ on each $P_{i}$. Let $\epsilon>0$ and let $A$ be a countable subset of $[a, b]$ that contains $\operatorname{Is}\left(\left\{P_{i}\right\}\right)$. Suppose that $A=\left\{a_{1}, a_{2}, \ldots, a_{i}, \ldots\right\}$. Since $F \in$ 
$\left(\mathcal{S}_{1} ; \mathcal{S}_{2}\right) \mathcal{C}$ on $[a, b]$, it follows that there exists $\beta_{A}=\beta_{A}\left(\sigma_{x}^{(1)}, \sigma_{x}^{(2)}\right) \in \mathcal{B}_{A}\left(\mathcal{S}_{1} ; \mathcal{S}_{2}\right)$ such that $\left|F(x)-F\left(a_{i}\right)\right|<\epsilon / 2^{i}$ whenever $x \in\left(\sigma_{a_{i}}^{(2)} \cap\left(a, a_{i}\right]\right) \cup\left(\sigma_{a_{i}}^{(1)} \cap\left[a_{i}, b\right)\right)$. Let $G_{1}, G_{2}:[a, b] \rightarrow \mathbb{R}, G_{1}(a)=G_{2}(a)=0$,

$$
\left.G_{1}(x)=\sum_{a_{i}<x} \mathcal{O}\left(F ;\left[a_{i}, b\right) \cap \sigma_{a_{i}}^{(1)}\right) \text { and } G_{2}(x)=\sum_{a_{i} \leq x} \mathcal{O}\left(F ;\left(a, a_{i}\right]\right) \cap \sigma_{a_{i}}^{(2)}\right) .
$$

(Here $\mathcal{O}(F ; X)$ denotes the oscillation of the function $F$ on the set $X$.) Clearly $G_{1}$ and $G_{2}$ are increasing on $[a, b], G_{1}(b)<\epsilon$ and $G_{2}(b)<\epsilon$. Fix some $a_{i}$. Then

$$
\left|F(x)-F\left(a_{i}\right)\right| \leq \mathcal{O}\left(F ;\left[a_{i} ; x\right] \cap \sigma_{a_{i}}^{(1)}\right)<G_{1}(x)-G_{1}\left(a_{i}\right),
$$

whenever $x \in\left[a_{i}, b\right) \cap \sigma_{a_{i}}^{(1)}$, and

$$
\left|F(x)-F\left(a_{i}\right)\right| \leq \mathcal{O}\left(F ;\left[x, a_{i}\right] \cap \sigma_{a_{i}}^{(2)}\right)<G_{2}\left(a_{i}\right)-G_{2}(x),
$$

whenever $x \in\left(a, a_{i}\right] \cap \sigma_{a_{i}}^{(2)}$. Let $S_{i}=\left\{x \in P_{i}: F_{P_{i}}^{\prime}(x)=f(x)\right\}$. Then $S_{i}$ is measurable and $\left|S_{i}\right|=\left|P_{i}\right|$ (see Lemma 10.2). Let $\delta_{i}: S_{i} \rightarrow(0,+\infty)$ such that

$$
|F(y)-F(x)-f(x)(y-x)|<\frac{\epsilon}{2(b-a)}|y-x|,
$$

whenever $y \in P_{i} \cap\left(x-\delta_{i}(x), x+\delta_{i}(x)\right)$. Let $G_{3}(x)=\epsilon(x-a) /(2(b-a))$. For $\epsilon / 2^{i}$ let $\eta_{i}>0$ be given by the fact that $F \in A C$ on $P_{i}$. Let $B_{i}=P_{i} \backslash S_{i}$. Then $\left|B_{i}\right|=0$, hence there exists an open set $G_{i}$ such that $B_{i} \subset G_{i}$ and $\left|G_{i}\right|<\eta_{i}$. Let $C=A \cup\left(\cup_{i=1}^{\infty} B_{i}\right)$. Then $|C|=0$. By the Tolstoff- Zahorski Theorem (see for example Theorem 2.14.6 of [1]), there exists $G_{4}:[a, b] \rightarrow(0,+\infty)$ such that $G_{4}$ is increasing on $[a, b], G_{4}(a)=0, G_{4}(b)<\epsilon$ and $G_{4}^{\prime}(x)=+\infty$ whenever $x \in C$. Let $\delta: C \rightarrow(0,+\infty)$ such that $\left(G_{4}(y)-G_{4}(x)\right) /(y-x)>|f(x)|$ whenever $y \in(x-\delta(x), x+\delta(x))$. Let $\sigma_{x}^{(k) \star}=\sigma_{x}^{(k)} \cap(x-\delta(x), x+\delta(x)), k=1,2$, $x \in A$. By (11) we obtain that $\left|F(x)-F\left(a_{i}\right)-f\left(a_{i}\right)\left(x-a_{i}\right)\right| \leq G_{1}(x)-G_{1}\left(a_{i}\right)+$ $G_{4}(x)-G_{4}\left(a_{i}\right)$, whenever $x \in\left[a_{i}, b\right) \cap \sigma_{x}^{(1) \star}$ and $\mid F(x)-F\left(a_{i}\right)-f\left(a_{i}\right)(x-$ $\left.a_{i}\right) \mid \leq G_{2}(x)-G_{2}\left(a_{i}\right)+G_{4}(x)-G_{4}\left(a_{i}\right)$, whenever $x \in\left(a, a_{i}\right] \cap \sigma_{x}^{(2) \star}$. Let $\delta_{i}: B_{i} \rightarrow(0,+\infty)$ such that $\delta_{i}(x)<\delta(x)$ and $\left.\left(x-\delta_{i}(x), x+\delta_{i}(x)\right) \subset G_{i}\right)$. Let $G_{5}(x)=\sum_{i=1}^{\infty} V\left(F ; B_{i} \cap[a, x]\right)$. Then $G_{5}(a)=0, G_{5}(b)<\sum_{i=1}^{\infty} \epsilon / 2^{i}=\epsilon$. We have $|F(y)-F(x)-f(x)(y-x)| \leq V\left(F ; B_{i} \cap[x, y]\right)+G_{4}(y)-G_{4}(x) \leq G_{5}(y)-$ $G_{5}(x)+G_{4}(y)-G_{4}(x)$, whenever $x \in B_{i}$ and $y \in P_{i} \cap\left(x-\delta_{i}(x), x+\delta_{i}(x)\right)$. Let $G=\sum_{i=1}^{5} G_{i}$ and let $\beta=\beta\left(\left\{P_{i}\right\},\left\{\delta_{i}\right\}\right) \in \overline{\mathcal{B}}_{[a, b]}$. It follows that $\mid F(y)-$ $F(x)-f(t)(y-x) \mid<G(y)-G(x)$ whenever $([x, y], t) \in \beta \cup \beta_{A}$. Therefore $F$ is the $\left[\mathcal{S}_{1} \mathcal{S}_{2} \mathcal{V}\right]$-indefinite integral of $f$ on $[a, b]$. Hence $\left[\mathcal{S}_{1} \mathcal{S}_{2} \mathcal{D}\right] \int_{a}^{b} f(t) d t=$ $\left[\mathcal{S}_{1} \mathcal{S}_{2} \mathcal{V}\right] \int_{a}^{b} f(t) d t$. 
Lemma 10.3. Let $\mathcal{S}_{1}=\left\{\mathcal{S}_{1}(x)\right\}_{x \in \mathbb{R}}$ be a local system $\mathcal{S}_{\infty}^{+}$-filtering on $[a, b)$, and let $\mathcal{S}_{2}=\left\{\mathcal{S}_{2}(x)\right\}_{x \in \mathbb{R}}$ be a local system $\mathcal{S}_{\infty}^{-}$-filtering on $(a, b]$. Let $f$ : $[a, b] \rightarrow \mathbb{R}$ be $\left[\mathcal{S}_{1} \mathcal{S}_{2} \mathcal{V}\right]$-integrable on $[a, b]$.

(i) (Saks-Henstock type lemma) For $\epsilon>0$ there is a $\beta=\beta\left(\left\{X_{i}\right\},\left\{\delta_{i}\right\}\right)$ $\in \overline{\mathcal{B}}_{[a, b]}$ with the following property: for every countable subset $A$ of $[a, b]$ that contains $I s\left(\left\{X_{i}\right\}\right)$ there is a $\beta_{A}=\beta_{A}\left(\sigma_{x}^{1)}, \sigma_{x}^{(2)}\right) \in \mathcal{B}_{A}\left(\mathcal{S}_{1} \mathcal{S}_{2}\right)$ such that

$$
\sum_{([x, y], t) \in \pi}\left|\left[\mathcal{S}_{1} \mathcal{S}_{2} \mathcal{V}\right] \int_{x}^{y} f(t) d t-f(t)(y-x)\right|<\epsilon,
$$

whenever $\pi$ is a $\left(\beta \cup \beta_{A}\right)$-partial partition of $[a, b]$.

(ii) $f$ is $\left[\mathcal{S}_{1} \mathcal{S}_{2} \mathcal{R}\right]$-integrable on $[a, b]$, and the $\left[\mathcal{S}_{1} \mathcal{S}_{2} \mathcal{V}\right]$ and $\left[\mathcal{S}_{1} \mathcal{S}_{2} \mathcal{R}\right]$ integrals are equal.

(iii) If $F(x)=\left[\mathcal{S}_{1} \mathcal{S}_{2} \mathcal{V}\right] \int_{a}^{x} f(t) d t$ then $F$ is $\left(\mathcal{S}_{1} ; \mathcal{S}_{2}\right) \mathcal{C}$ on $[a, b]$.

Proof. (i) Since $f$ is $\left[\mathcal{S}_{1} \mathcal{S}_{2} \mathcal{V}\right]$-integrable on $[a, b]$, there exists $H:[a, b] \rightarrow \mathbb{R}$ such that for every $\epsilon>0$ there exists a $\beta=\beta\left(\left\{X_{i}\right\},\left\{\delta_{i}\right\}\right) \in \overline{\mathcal{B}}_{[a, b]}$ with the following property: for every countable subset $A$ of $[a, b]$ that contains $\operatorname{Is}\left(\left\{X_{i}\right\}\right)$ there is a $\beta_{A}=\beta_{A}\left(\sigma_{x}^{1)}, \sigma_{x}^{(2)}\right) \in \mathcal{B}_{A}\left(\mathcal{S}_{1} ; \mathcal{S}_{2}\right)$ and there exists an increasing function $G:[a, b] \rightarrow[0,+\infty)$, such that $G(a)=0, G(b)<\epsilon$ and $\mid H(y)-H(x)-$ $f(t)(y-x) \mid<G(y)-G(x)$, whenever $([x, y], t) \in \beta \cup \beta_{A}$. Let $\pi$ be a $\left(\beta \cup \beta_{A}\right)$ -

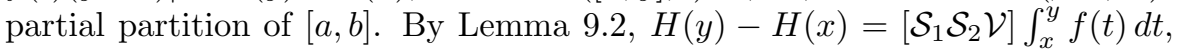
hence

$$
\begin{gathered}
\sum_{([x, y], t) \in \pi}\left|f(t)(y-x)-\left[\mathcal{S}_{1} \mathcal{S}_{2} \mathcal{V}\right] \int_{x}^{y} f(t) d t\right|= \\
\sum_{([x, y], t) \in \pi}|f(t)(y-x)-(H(y)-H(x))| \leq \sum_{([x, y], t) \in \pi}(G(y)-G(x)) \leq G(b)<\epsilon .
\end{gathered}
$$

(ii) With the notations of (i), let $\pi$ be a $\left(\beta \cup \beta_{A}\right)$-partition of $[a, b]$. Then

$$
\begin{aligned}
& |s(f ; \pi)-(H(b)-H(a))|=\left|\sum_{([x, y], t) \in \pi}(f(t)(y-x)-H(y)+H(x))\right| \\
\leq & \sum_{([x, y], t) \in \pi}|f(t)(y-x)-H(y)+H(x)| \leq \sum_{([x, y], t) \in \pi}(G(y)-G(x))<\epsilon .
\end{aligned}
$$

It follows that $f$ is $\left[\mathcal{S}_{1} \mathcal{S}_{2} \mathcal{R}\right]$-integrable on $[a, b]$, and then

$$
\left[\mathcal{S}_{1} \mathcal{S}_{2} \mathcal{R}\right] \int_{a}^{b} f(t) d t=H(b)-H(a)=\left[\mathcal{S}_{1} \mathcal{S}_{2} \mathcal{V}\right] \int_{a}^{b} f(t) d t
$$


(iii) Let $x_{o} \in[a, b)$ and $\epsilon>0$. Let $\delta>0$ such that $\left|f\left(x_{o}\right)\right| \cdot \delta<\epsilon$. For $\epsilon$ let $\beta=\beta\left(\left\{X_{i}\right\},\left\{\delta_{i}\right\}\right) \in \overline{\mathcal{B}}_{[a, b]}$ be given by (i). Let $A=\left\{x_{o}\right\} \cup \operatorname{Is}\left(\left\{X_{i}\right\}\right.$. Then for every $x \in \sigma_{x_{o}}^{(1)} \in \mathcal{S}_{1}\left(x_{o}\right), x>x_{o}$ we have $\left|F(x)-F\left(x_{o}\right)\right| \leq \mid F(x)-F\left(x_{o}\right)-$ $f\left(x_{o}\right)\left(x-x_{o}\right)|+| f\left(x_{o}\right) \mid\left(x-x_{o}\right)<2 \epsilon$. It follows that $F$ is right $\mathcal{S}_{1}$-continuous on $[a, b)$. Similarly we obtain that $F$ is left $\mathcal{S}_{2}$-continuous on $(a, b]$. By Lemma $2.1,(\mathrm{i}), F$ is $\left(\mathcal{S}_{1} ; \mathcal{S}_{2}\right) \mathcal{C}$ on $[a, b]$.

\section{The Characterization of the $\mathcal{D}$-Integral}

Theorem 11.1. Let $f:[a, b] \rightarrow \mathbb{R}$. The following assertions are equivalent:

(i) $f$ is $\left[\mathcal{S}_{o}^{+} \mathcal{S}_{o}^{-} \mathcal{D}\right]$-integrable (i.e., $\mathcal{D}$-integrable) on $[a, b]$;

(ii) $f$ is $\left[\mathcal{S}_{o}^{+} \mathcal{S}_{o}^{-} \mathcal{V}\right]$ - integrable on $[a, b]$;

(iii) $f$ is $\left[\mathcal{S}_{o}^{+} \mathcal{S}_{o}^{-} \mathcal{W}\right]$-integrable on $[a, b]$.

(iv) $f$ is $\left[\mathcal{S}_{o}^{+} \mathcal{S}_{o}^{-} \mathcal{R}\right]$-integrable on $[a, b]$ and $F$ is continuous on $[a, b]$, where $F(x)=\left[\mathcal{S}_{o}^{+} \mathcal{S}_{o}^{-} \mathcal{R}\right] \int_{a}^{x} f(t) d t$.

Moreover, all the integrals are equal.

Proof. (i) $\Rightarrow$ (ii) and the equality of the integrals follow by Theorem 10.1.

(ii) $\Leftrightarrow$ (iii) and the equality of the integrals follow by Lemma 10.1.

(ii) $\Rightarrow$ (iv) and the equality of the integrals follow by Lemma 10.3.

(iv) $\Rightarrow\left(\right.$ i) By hypothesis $F(x)=\left[\mathcal{S}_{o}^{+} \mathcal{S}_{o}^{-} \mathcal{R}\right] \int_{a}^{x} f(t) d t$ is continuous on $[a, b]$. By Corollary 7.1, $F \in(N)$ on $[a, b]$, and by Lemma 7.6, $F$ is $V B G$ on $[a, b]$. Therefore $F \in \mathcal{C} \cap V B G \cap(N)=\mathcal{C} \cap A C G$ on $[a, b]$ (see for example [1, p. $75])$. Now, by Lemma $7.5, F_{a p}^{\prime}(x)=f(x)$ a.e. on $[a, b]$. It follows that $f$ is $\mathcal{D}$-integrable on $[a, b]$, and the two integrals are equal.

Remark 11.1. The fact that $F \in A C G$ in the proof of Theorem 11.1, (iv) $\Rightarrow$ (i), can also be obtained as follows: by Corollary $7.1, F \in N_{\mathcal{B}_{o}}$ on $[a, b]$; and by Theorem 5.2, $F \in A C G$ on $[a, b]$.

\section{Query}

Definition 12.1. Let $\mathcal{S}_{1}=\left\{\mathcal{S}_{1}(x)\right\}_{x \in \mathbb{R}}$ be a local system $\mathcal{S}_{\infty}^{+}$-filtering on $[a, b)$, and let $\mathcal{S}_{2}=\left\{\mathcal{S}_{2}(x)\right\}_{x \in \mathbb{R}}$ be a local system $\mathcal{S}_{\infty}^{-}$-filtering on $(a, b]$. A function $f:[a, b] \rightarrow \mathbb{R}$ is said to be strong $\left[\mathcal{S}_{1} \mathcal{S}_{1} \mathcal{D}\right]$ integrable on $[a, b]$ if there exists a function $F:[a, b] \rightarrow \mathbb{R}$ with the following properties:

(i) $F \in\left(\mathcal{S}_{1} ; \mathcal{S}_{2}\right) \mathcal{C}$; 
(ii) $F \in[V B G] \cap(N)$;

(iii) $F_{a p}^{\prime}(x)=f(x)$ a.e. on $[a, b]$.

We write strong $\left[\mathcal{S}_{1} \mathcal{S}_{1} \mathcal{D}\right] \int_{a}^{b} f(t) d t=F(b)-F(a)$.

Remark 12.1. Note that Lemma 7.2 remains true if condition $[\underline{A C G}]$ is replaced by $[V B G] \cap(N)$, and Corollary 2.1 remains true if $[A C G]$ is replaced by $[V B G] \cap(N)$. But $[V B G] \cap(N)$ is a real linear space on $[a, b]$ (see Corollary 3.1.1 and Theorem 3.6 of [19]). It follows that the above integral is well defined.

Question. How can the definitions of $\left[\mathcal{S}_{1} \mathcal{S}_{2} \mathcal{V}\right]$, $\left[\mathcal{S}_{1} \mathcal{S}_{2} \mathcal{W}\right]$ and $\left[\mathcal{S}_{1} \mathcal{S}_{2} \mathcal{R}\right]$ be modified such that each of them contain the strong $\left[\mathcal{S}_{1} \mathcal{S}_{2} \mathcal{D}\right]$ - integral?

Remark 12.2. Definition 12.1 can be extended by replacing condition (ii) with " $F \in V B G \cap(N) \cap \mathcal{B}_{1}$ ". This is so because Lemma 7.2 still remains true if $[\underline{A C G}]$ is replaced by $V B G \cap(N) \cap \mathcal{B}_{1}$, Corollary 2.1 still remains true if $[A C G]$ is replaced by $V B G \cap(N) \cap \mathcal{B}_{1}$, and $V B G \cap(N) \cap \mathcal{B}_{1}$ is a real linear space (the proof of the latter is not easy, and it is shown by the author in [2]).

A special case of this new definition is an integral defined by Gordon in [4] (Definition 3): (i) is replaced by " $F \in \mathcal{C}_{a p}$ ", and (ii) by " $F \in V B G \cap(N)$ ". However his argumentation about the integral being well defined is incomplete, because he doesn't take in consideration whether $V B G \cap(N) \cap \mathcal{C}_{a p}$ is a linear space or not.

\section{Appendix}

* After this paper has been accepted for publication in the present journal, the author withdrew it, having in mind a lot of revisions. The present paper contains a lot of them, but his main intention was to give up the two local systems in the definitions of the general integrals, and use instead only one. He had started to do so but never finished his work. In what follows the revised statements (with proofs) will be given as well as the changed definitions. The modified results will be labelled with the old numbers and a 'prime' sign.

Lemma 2.1'. Let $\mathcal{S}=\{\mathcal{S}(x)\}_{x \in \mathbb{R}}$ be a bilateral local system satisfying the following property for each $x \in \mathbb{R}$ :

$$
\text { If } \sigma_{x}^{\prime}, \sigma_{x}^{\prime \prime} \in \mathcal{S}(x) \text { then }\left(\sigma_{x}^{\prime} \cap(-\infty, x]\right) \cup\left(\sigma_{x}^{\prime \prime} \cap[x,+\infty)\right) \in \mathcal{S}(x) .
$$

Let $F:[a, b] \rightarrow \mathbb{R}$. Then we have:

(i) $F$ is $\mathcal{S C}$ on $[a, b]$ if and only if it is bilaterally $\mathcal{S C}$ on $[a, b]$.

(ii) $F$ is $\mathcal{S C}_{i}$ on $[a, b]$ if and only if $F$ is simultaneously right $\mathcal{S}$-lower semi-continuous on $[a, b)$ and left $\mathcal{S}$-upper semi-continuous on $(a, b]$.

Proof. Evident.

*Extracted by Gabriela Ene from the author's notes. 


\section{4' A Fundamental Lemma}

Following the notations in [21] (pp. 5,6), we shall denote by $\mathcal{I}$ the collection of all nondegenerate, real compact intervals. A subset $\beta$ of the product $\mathcal{I} \times \mathbb{R}$ is said to be a covering relation if $x \in I$ whenever $(I, x) \in \beta$ (see [22, p. 5]). If $\beta$ is a covering relation and $E$ a real set then $\beta(E), \beta[E]$ and $\sigma(\beta)$ denote the following sets:

- $\beta(E)=\{(I, x) \in \beta: I \subset E\}$;

- $\beta[E]=\{(I, x) \in \beta: x \in E\}$;

- $\sigma(\beta)=\cup_{(I, x) \in \beta} I$.

A packing is a covering relation $\beta$ with the property that for distinct pairs $\left(I_{1}, x_{1}\right)$ and $\left(I_{2}, x_{2}\right)$ the intervals $I_{1}$ and $I_{2}$ do not overlap. Evidently a packing is either finite or countable infinite. Using the language of Henstock we call a finite packing $\beta$ a division (a $\beta$-division) of an interval $[a, b]$ if $\sigma(\beta)=[a, b]$.

Definition 13.1. Let $E$ be a real set and $\delta: E \rightarrow(0,+\infty)$. We denote by $\beta(E ; \delta)=$ $\{(\langle x, y\rangle): x, y \in E, x$ is an accumulation point for $\langle x, y\rangle \cap E$, and $\langle x, y\rangle \subset(x-\delta(x), x+\delta(x)\}$. Clearly $\beta(E ; \delta)$ is a covering relation (possibly empty).

Definition $4.2^{\prime}$. Let $P$ be a real set. We denote by

- $\mathrm{Is}^{+}(P)=\{x \in P: x$ is a right isolated point of $P\}$

- $\mathrm{Is}^{-}(P)=\{x \in P: x$ is a left isolated point of $P\}$;

- $\mathrm{Is}(P)=\mathrm{Is}^{+}(P) \cup \mathrm{Is}^{-}(P)$. This set is countable (see [15, p. 260]).

Definition 13.2. ([16]). A sequence $\left\{E_{n}\right\}$ of sets whose union is $E$ is called an $E$-form with parts $E_{n}$. If, in addition, each part $E_{n}$ is closed in $E$ (i.e., $E_{n}=P_{n} \cap E$, where $P_{n}$ is a closed set, so $P_{n}=\bar{E}_{n}$ ) then the $E$-form is said to be closed.

Definition 13.3. Let $\left\{E_{i}\right\}_{i}$ be a closed $[a, b]$-form, $\delta_{i}: E_{i} \rightarrow(0,+\infty)$, and $A$ a set that contains $\cup_{i=1}^{\infty} \operatorname{Is}\left(E_{i}\right)$. For each $a \in A$ let $\sigma_{a}$ be a set having the point $a$ as a bilateral accumulation point. Let

$$
\beta=\beta\left(\left\{E_{i}\right\} ;\left\{\delta_{i}\right\} ;\left(\sigma_{a}\right)_{a \in A}\right)=\cup_{i=1}^{\infty} \beta\left(E_{i} ; \delta_{i}\right) \cup\left(\cup_{a \in A}\left\{(\langle a, x\rangle, a): x \in \sigma_{a} \backslash\{a\}\right\}\right) .
$$

Clearly $\beta$ is a covering relation. It contains the $A D$ full cover of Lee and Soedijono (see [12, p. 265]), the cover $\mathcal{U}$ of Henstock (see [7, p. 56]), the covering relation called "composite path derivation" defined by Thomson (see [20, p. 104]), and Henstock's covering relation $P C$ ([20, p. 115]).

Lemma 4.1' . Let $P$ be a perfect nowhere dense subset of $[a, b], a, b \in P$, and let $\delta: P \rightarrow$ $(0,+\infty)$. Then there exists a finite packing $\pi$ contained in $\beta(P ; \delta)$ such that $\sigma(\pi) \supset P$.

Proof. Let $\left\{\left(a_{i}, b_{i}\right)\right\}, i=\overline{1, \infty}$ be the intervals contiguous to $P$, and let $\eta:[a, b] \rightarrow(0,+\infty)$,

$$
\eta(x)=\left\{\begin{array}{llll}
\delta(x) & , & \text { if } \quad x \in P \backslash \cup_{i=1}^{\infty}\left\{a_{i}, b_{i}\right\} & \\
\min \left\{\frac{b_{i}-a_{i}}{3}, \delta(x)\right\} & , & \text { if } \quad x \in\left\{a_{i}, b_{i}\right\} & , \\
\min \left\{\frac{x-a_{i}}{2}, \frac{b_{i}-x}{2}\right\} & , & \text { if } \quad x \in\left(a_{i}, b_{i}\right) & , \quad i=\overline{1, \infty}
\end{array}\right.
$$

Let $\pi$ be a $\beta([a, b], \eta)$ partition of $[a, b]$ (that such a partition exists follows for example by $\left[1\right.$, p. 87]). Let $\pi=\left\{\left(\left\langle x_{i}, y_{i}\right\rangle, x_{i}\right)\right\}_{i=1}^{n}$ and $\mathcal{A}=\left\{i \in\{1,2, \ldots, n\}: \operatorname{int}\left(\left\langle x_{i}, y_{i}\right\rangle\right) \cap P \neq \emptyset\right\}$ (here $\operatorname{int}(X)$ denotes the interior of the set $X$ ). For $x \in P \backslash \cup_{i=1}^{\infty}\left\{a_{i}, b_{i}\right\}$, it follows that 
$x \in\left\langle x_{i}, y_{i}\right\rangle$ for some $i \in\{1,2, \ldots, n\}$. Clearly $\left\langle x_{i}, y_{i}\right\rangle \cap P$ is an infinite set, so $i \in \mathcal{A}$. Therefore

$$
P \backslash\left(\cup_{i=1}^{\infty}\left\{a_{i}, b_{i}\right\}\right) \subset \cup_{i \in \mathcal{A}}\left\langle x_{i}, y_{i}\right\rangle .
$$

It follows that

$$
P=\overline{P \backslash\left(\cup_{i=1}^{\infty}\left\{a_{i}, b_{i}\right\}\right)} \subset \cup_{i \in \mathcal{A}}\left\langle x_{i}, y_{i}\right\rangle .
$$

If $i \in \mathcal{A}$ then $x_{i} \in P$ (because if $x_{i} \in\left(a_{j}, b_{j}\right)$ for some $j$ then $y_{i} \in\left(x_{i}-\eta\left(x_{i}\right), x_{i}+\eta\left(x_{i}\right)\right) \subset$ $\left(a_{j}, b_{j}\right)$, which implies that $i \notin \mathcal{A}$, a contradiction).

Fix some $i \in \mathcal{A}$. If $x_{i} \in P \backslash\left(\cup_{j=1}^{\infty}\left\{a_{j}, b_{j}\right\}\right)$ then $x_{i}$ is an accumulation point for $\left\langle x_{i}, y_{i}\right\rangle \cap P$. Let $z_{i} \in P$ such that $\left\langle x_{i}, y_{i}\right\rangle \cap P=\left\langle x_{i}, z_{i}\right\rangle \cap P$. Then $\left(\left\langle x_{i}, z_{i}\right\rangle, x_{i}\right) \in \beta(P ; \delta)$.

If $x_{i}=a_{j}$ for some $j$ then $y_{i}<x_{i}$ (because if $x_{i}<y_{i}$ then $\left[x_{i}, y_{i}\right] \subset\left[a_{j}+\frac{b_{j}-a_{j}}{3}\right]$, so $i \notin \mathcal{A}$ ). It follows that $\left[y_{i}, x_{i}\right] \cap P$ has $x_{i}$ as an accumulation point. Let $z_{i}=\inf \left[y_{i}, x_{i}\right] \cap P$, hence $\left[y_{i}, x_{i}\right] \cap P=\left[z_{i}, x_{i}\right] \cap P$. Consequently $\left(\left[z_{i}, x_{i}\right], x_{i}\right) \in \beta(P ; \delta)$.

If $x_{i}=b_{j}$ for some $j$, then $y_{i}>x_{i}$. Let $z_{i}=\sup \left(P \cap\left[x_{i}, y_{i}\right]\right)$. Then $\left(\left[x_{i}, z_{i}\right], x_{i}\right) \in \beta(P ; \delta)$.

By (13), it follows easily that $\pi=\left\{\left(\left\langle x_{i}, z_{i}\right\rangle, x_{i}\right)\right\}_{i \in \mathcal{A}}$ satisfies our lemma.

Lemma 4.2' (Fundamental lemma). For each $\beta=\beta\left(\left\{E_{i}\right\} ;\left\{\delta_{i}\right\} ;\left(\sigma_{a}\right)_{a \in \mathcal{A}}\right)$, the interval $[a, b]$ has a $\beta$-division.

Proof. We shall use the Romanovski Lemma (see for example [1, p. 10]). Let $\mathcal{A}=\{(p, q) \subseteq$ $(a, b):\left[p_{1}, q_{1}\right]$ has a $\beta$-division whenever $\left.\left(p_{1}, q_{1}\right) \subseteq(p, q)\right\}$.

(i) If $(p, q) \in \mathcal{A}$ and $(q, r) \in \mathcal{A}$ then clearly $(p, r) \in \mathcal{A}$.

(ii) If $(p, q) \in \mathcal{A}$ and $\left(p_{1}, q_{1}\right) \subset(p, q)$ then $\left(p_{1}, q_{1}\right) \in \mathcal{A}$ (see the definition of $\left.\mathcal{A}\right)$.

(iii) Let $(c, d) \subseteq(a, b)$ such that $(p, q) \in \mathcal{A}$ whenever $[p, q] \subset(c, d)$. We show that $(c, d) \in \mathcal{A}$. Let $c \in E_{n}$. Let $c_{1} \in\left(c, c+\delta_{n}(c)\right) \cap E_{n} \cap(c,(c+d) / 2)$ if $c$ is a right accumulation point for $E_{n}$, and let $c_{1} \in \sigma_{c} \cap(c,(c+d) / 2)$ if $c$ is right isolated in $[a, b) \cap E_{n}$. Then $\left(\left[c, c_{1}\right], c\right) \in \beta$. Similarly we find $d_{1} \in((c+d) / 2, d)$ such that $\left(\left[d_{1}, d\right], d\right) \in \beta$. But $\left(c_{1}, d_{1}\right) \in \mathcal{A}$ and $[c, d]=\left[c, c_{1}\right] \cup\left[c_{1}, d_{1}\right] \cup\left[d_{1}, d\right]$. Therefore $[c, d]$ admits a $\beta$-division. Analogously we obtain that $\left[c_{2}, d_{2}\right]$ has a $\beta$-division, whenever $\left(c_{2}, d_{2}\right) \subset(c, d)$. Hence $(c, d) \in \mathcal{A}$.

(iv) Let $E \subset[a, b]$ be a perfect set such that all intervals contiguous to $E$ are contained in $\mathcal{A}$. We show that there exists $(p, q) \in \mathcal{A}$ such that $E \cap(p, q) \neq \emptyset$. Since $E=\cup_{n=1}^{\infty}\left(E \cap E_{n}\right)$, by the Baire Category Theorem (see for example [1, p. 10]) it follows that there exists a positive integer $n$ and an interval $(p, q)$ such that $\emptyset \neq(p, q) \cap E=\left(E \cap E_{n}\right) \cap(p, q)$. We may suppose without loss of generality that $p, q \in E$ and $[p, q] \cap E$ is perfect. Applying Lemma $4.1^{\prime}$ to $[p, q] \cap E$ and $\delta_{n}$, there exists a finite packing $\pi$ contained in $\beta\left(E ; \delta_{n}\right)$ such that $\sigma(\pi) \supset E$. Clearly $\pi$ is a finite packing contained in $\beta$. Since $[p, q] \backslash \sigma(\pi)$ consists of a finite number of intervals contiguous to $E$, it follows that $[p, q]$ admits a $\beta$-division. Similarly it follows that each $\left[p_{1}, q_{1}\right]$ admits such a division, whenever $\left(p_{1}, q_{1}\right) \subset(p, q)$. Therefore $(p, q) \in \mathcal{A}$.

By (i)-(iv) and the Romanovski Lemma, it follows that $(a, b) \in \mathcal{A}$.

\section{$6^{\prime}$ The Lusin Type $[\mathcal{S D}]$ Integral}

Definition 6.1'. Let $\mathcal{S}=\{\mathcal{S}(x)\}_{x \in \mathbb{R}}$ be a local system $\mathcal{S}_{\infty, \infty}$-filtering. Let $f: \mathbb{R} \rightarrow \overline{\mathbb{R}}$, and let $E$ a bounded nonempty set, with $a=\inf E, b=\sup E$. $f$ is said to be $[\mathcal{S D}]$-integrable on $E$ if there is a real number $I$ and a function $F: \mathbb{R} \rightarrow \mathbb{R}$,

$$
F(x)= \begin{cases}0 & \text { if } x \leq a \\ I & \text { if } x \geq b\end{cases}
$$


such that $F$ is $\mathcal{S C}$ on $[a, b], F \in[A C G]$ on $[a, b]$, and $F_{a p}^{\prime}(x)=\chi_{E}(x) \cdot f(x)$ a.e. on $[a, b]$, where $\chi_{E}$ is the characteristic function of $E$. We write $[\mathcal{S D}] \int_{E} f(t) d t=I . F$ is said to be the (unique) indefinite integral of $f$ on $E$.

Lemma 6.2'. Let $f: \mathbb{R} \rightarrow \overline{\mathbb{R}}$ be $[\mathcal{S D}]$-integrable on $[a, b]$ and let $c \in(a, b)$. Then $f$ is $[\mathcal{S D}]$-integrable on both $[a, c]$ and $[a, d]$, and we have

$$
[\mathcal{S D}] \int_{a}^{b} f(t) d t=[\mathcal{S D}] \int_{a}^{c} f(t) d t+[\mathcal{S D}] \int_{c}^{b} f(t) d t
$$

Proof. Let $F: \mathbb{R} \rightarrow \mathbb{R}$ be the indefinite $[\mathcal{S D}]$-integral of $f$ on $[a, b]$. Let $F_{1}, F_{2}: \mathbb{R} \rightarrow \mathbb{R}$,

$$
F_{1}(x)=\left\{\begin{array}{ll}
F(x) & \text { if } x \in(-\infty, c] \\
F(c) & \text { if } x \in[c,+\infty)
\end{array}, \quad F_{2}(x)= \begin{cases}0 & \text { if } x \in(-\infty, c] \\
F(x)-F(c) & \text { if } x \in[c,+\infty) .\end{cases}\right.
$$

Then $F_{1}$ (respectively $F_{2}$ ) is the indefinite $[\mathcal{S D}]$-integral of $f$ on $[a, c]$ (respectively $[c, b]$ ) and we have the relation from above.

Lemma 6.3'. Let $f: \mathbb{R} \rightarrow \overline{\mathbb{R}}$ be $[\mathcal{S D}]$-integrable on $[a, c]$ and on $[c, b]$, where $c \in(a, b)$. Then $f$ is $[\mathcal{S D}]$-integrable on $[a, b]$ and we have:

$$
[\mathcal{S D}] \int_{a}^{b} f(t) d t=[\mathcal{S D}] \int_{a}^{c} f(t) d t+[\mathcal{S D}] \int_{c}^{b} f(t) d t .
$$

Proof. Let $F_{1}$ (respectively $F_{2}$ ) be the indefinite $[S \mathcal{S D}$-integral of $f$ on $[a, c]$ (respectively $[c, b])$. Then $F=F_{1}+F_{2}$ is the indefinite $[\mathcal{S D}]$-integral of $f$ on $[a, b]$ and we have the relation from above.

\section{$7^{\prime}$ The Riemann Type $[\mathcal{S R}]$ Integral}

Definition 7.1'. Let $\mathcal{S}=\{\mathcal{S}(x)\}) x \in \mathbb{R}$ be a local system $\mathcal{S}_{\infty, \infty}$-filtering. A function $f: \mathbb{R} \rightarrow \mathbb{R}$ is said to be $[\mathcal{S R}]$ integrable on $[a, b]$ to $I \in \mathbb{R}$ if for $\epsilon>0$ there exist a closed $[a, b]$-form $\left\{E_{i}\right\}, \delta_{i}: E_{i} \rightarrow(0,+\infty)$, for $A \supseteq \cup_{i=1}^{\infty} \operatorname{Is}\left(E_{i}\right)$ countable there is $\Delta_{A, \epsilon}: A \rightarrow \mathcal{P}(\mathbb{R})$, $\Delta_{A, \epsilon}(x) \in \mathcal{S}(x)$, and for $B \supset A, B$ countable there is a $\Delta_{B, \epsilon}$ such that

$$
|s(f ; \alpha)-I|<\epsilon,
$$

whenever $\alpha \subset \beta\left(\left\{E_{i}^{\epsilon}\right\},\left\{\delta_{i}^{\epsilon}\right\}, \Delta_{A, \epsilon} \vee \Delta_{B \epsilon}\right)$ is a division of $[a, b]$.

Theorem 7.1'. The number I in Definition $7.1^{\prime}$ is unique, and it will be denoted by $[\mathcal{S R}] \int_{a}^{b} f(t) d t$.

Proof. Suppose that there exist two numbers $I_{1}$ and $I_{2}$ as in Definition 7.1'. Let $\left\{E_{i}^{k}\right\}_{i=1}^{\infty}$, $k=1,2$, be a closed $[a, b]$-form given for $I_{k}$ and $\epsilon$. Let $E_{i j}=E_{i}^{1} \cap E_{j}^{2}, \delta_{i j}^{k}=E_{i j} \rightarrow(0,+\infty)$ and $\Delta^{k}: \cup_{i, j} \operatorname{Is}\left(E_{i j}\right) \rightarrow \mathcal{P}(\mathbb{R}), \Delta_{k}(x) \in \mathcal{S}(x)$, be such that $\left|s\left(f ; \pi_{k}\right)-I-k\right|<\epsilon$, whenever $\pi_{k} \subset \beta_{k}=\beta\left(\left\{E_{i j}\right\} ; \delta_{i j}^{k} ; \Delta_{k}\right)$ is a division of $[a, b]$. Let $\delta_{i j}: E_{i j} \rightarrow(0,+\infty), \delta_{i j}(x)=$ $\min \left\{\delta_{i j}^{1}(x), \delta_{i j}^{2}(x)\right\}$, and let $\Delta: \cup_{i, j} \operatorname{Is}\left(E_{i j}\right) \rightarrow \mathcal{P}(\mathbb{R}), \Delta(x)=\Delta_{1}(x) \cap \Delta_{2}(x) \in \mathcal{S}_{\infty, \infty}(x)$. By Lemma $4.2^{\prime}$ there exists $\pi \subset \beta=\beta\left(\left\{E_{i j}\right\} ;\left\{\delta_{i j}\right\} ; \Delta\right)$. Clearly $\pi \subset \beta_{1} \cap \beta_{2}$. Hence $\left|s(f ; \pi)-I_{k}\right|<\epsilon, k=1,2$. It follows that $\left|I_{1}-I_{2}\right|<2 \epsilon$. Since $\epsilon$ is arbitrary we obtain that $I_{1}=I_{2}$.

Remark 13.1. If $\alpha \in \mathbb{R}$ and $f$ is $[\mathcal{S R}]$-integrable to $I$ on $[a, b]$ then $\alpha f$ is $[\mathcal{S R}]$-integrable to $\alpha I$ on $[a, b]$. 
Lemma 7.1'. Let $\mathcal{S}$ be a filtering local system on $[a, b]$. Let $f_{1}, f_{2}:[a, b] \rightarrow \mathbb{R}$ be $[\mathcal{S R}]$ integrable to $I_{1}$ respectively $I_{2}$ on $[a, b]$. Then $f_{1}+f_{2}$ is $[\mathcal{S R}]$-integrable on $[a, b]$ to $I_{1}+I_{2}$.

Proof. For $\frac{\epsilon}{2}>0$ and $I_{k}, k=1,2$, let $\left\{E_{i}^{k}\right\}$ be a closed $[a, b]$-form given by Definition 7.1'. Let $E_{i j}=E_{i}^{1} \cap E_{j}^{2}$. Then $\left\{E_{i j}\right\}_{i, j}$ is a closed $[a, b]$-form. Let $\left\{X_{i}\right\}$ be a closed $[a, b]$ form finer than $\left\{E_{i j}\right\}$. Clearly $\left\{X_{i}\right\}$ is finer than $\left\{E_{i}\right\}^{k}$. By Definition 7.1', there exist $\delta_{i}^{k}: X_{i} \rightarrow(0,+\infty)$ and $\Delta^{k}: \cup_{i} \operatorname{Is}\left(X_{i}\right) \rightarrow \mathcal{P}(\mathbb{R}), \Delta_{k}(x) \in \mathcal{S}(x)$ such that

$$
\left|s\left(f_{k} ; \pi_{k}\right)-I_{k}\right|<\frac{\epsilon}{2},
$$

whenever $\pi_{k} \subset \beta_{k}=\beta\left(\left\{X_{i}\right\} ;\left\{\delta_{i}^{k}\right\} ; \Delta_{k}\right)$ is a division of $[a, b]$. Let $\delta_{i}: X_{i} \rightarrow(0,+\infty)$, $\delta_{i}(x)=\min \left\{\delta_{i}^{1}(x), \delta_{i}^{2}(x)\right\}$, and let $\left.\Delta: \cup_{i} \operatorname{Is}\left(X_{i}\right) \rightarrow \mathcal{P}(\mathbb{R}), \Delta(x)=\Delta_{1}(x) \cap \Delta_{(} x\right) \in \mathcal{S}(x)$ (because $\mathcal{S}$ is filtering). Let $\pi \subset \beta=\beta\left(\left\{X_{i}\right\} ;\left\{\delta_{i}\right\} ; \Delta\right)$ be a division of $[a, b]$ (this is possible by Lemma $\left.4.2^{\prime}\right)$. Clearly $\pi$ is a $\beta_{k}$ division. Hence

$$
\left|s\left(f_{1}+f_{2} ; \pi\right)-\left(I_{1}+I_{2}\right)\right|<\left|s\left(f_{1} ; \pi\right)-I_{1}\right|+\left|s\left(f_{2} ; \pi\right)-I_{2}\right|<\epsilon .
$$

Thus $f_{1}+f_{2}$ is $[\mathcal{S R}]$-integrable to $I_{1}+I_{2}$ on $[a, b]$.

Lemma 7.2' (A Cauchy criterion). Let $\mathcal{S}=\{\mathcal{S}(x)\}) x \in \mathbb{R}$ be a local system $\mathcal{S}_{\infty, \infty}$-filtering on $[a, b]$, and let $f: \mathbb{R} \rightarrow \mathbb{R}$. The following assertions are equivalent:

(i) $f \in[\mathcal{S R}]$ integrable on $[a, b]$;

(ii) for $\epsilon>0$ there exist a closed $[a, b]$-form $\left\{E_{i}^{\epsilon}\right\}, \delta_{i}^{\epsilon}: E_{i}^{\epsilon} \rightarrow(0,+\infty)$, for $A \supseteq \cup_{i=1}^{\infty} I s\left(E_{i}^{\epsilon}\right)$ there is a $\Delta_{A, \epsilon}$ and for $B \supset A, B$ countable, there is a $\Delta_{B, \epsilon}$ such that

$$
\left|s\left(f, \pi_{1}\right)-s\left(f ; \pi_{2}\right)\right|<\epsilon,
$$

whenever $\pi_{1}, \pi_{2} \subset \beta\left(\left\{E_{i}^{\epsilon}\right\},\left\{\delta_{i}^{\epsilon}\right\}, \Delta_{A, \epsilon} \vee \Delta_{B, \epsilon}\right)$.

Proof. (i) $\Rightarrow$ (ii) This is obvious.

(ii) $\Rightarrow$ (i) For $\frac{1}{k}$ let $\left\{E_{i}^{\frac{1}{k}}\right\}$ be a closed $[a, b]$-form, $\delta^{\frac{1}{k}}: E_{i}^{\frac{1}{k}} \rightarrow(0,+\infty)$ be given by (ii). Let $A_{k}$ be countable, $A_{k} \supseteq \cup_{i=1}^{\infty} \operatorname{Is}\left(E_{i}^{\frac{1}{k}}\right)$ and $B_{k} \supset A_{k}, B_{k}$ countable. Let $A_{o}=\cup_{i=1}^{\infty} A_{k}$ and $B_{o}=\cup_{i=1}^{\infty} B_{k}$.Again by (ii), for $A_{o}$ there is a $\Delta_{A_{o}, \frac{1}{k}}$ and for $B_{o}$ there is a $\Delta_{B_{o}, \frac{1}{k}}$ such that

$$
\left|s\left(f ; \pi_{k}^{\prime}\right)-s\left(f ; \pi_{k}^{\prime \prime}\right)\right|<\frac{1}{k},
$$

whenever $\pi_{k}^{\prime}, \pi_{k}^{\prime \prime} \subset \beta_{k}=\beta\left(\left\{E_{i}^{\frac{1}{k}}\right\},\left\{\delta_{i}^{\frac{1}{k}}\right\}, \Delta_{A_{o}, \frac{1}{k}} \vee \Delta_{B_{o}, \frac{1}{k}}\right)$ are divisions of $[a, b]$. Let $\pi_{k} \subset$ $\beta_{k}^{*}=\beta\left(\left\{E_{i}^{\frac{1}{k}}\right\},\left\{\delta_{i}^{\frac{1}{k}}\right\}, \Delta_{A_{k}, \frac{1}{k}}\right) \subset \beta_{k}$ be a fixed division of $[a, b]$, where $\Delta_{A_{k}, \frac{1}{k}}=\Delta_{A_{o}, \frac{1}{k}} / A_{k}$. Let $\epsilon>0$ and let $k_{\epsilon}$ be a positive integer such that $1 / k_{\epsilon}<\epsilon / 2$. Let $k_{\epsilon} \leq m<n$. Let

$$
\pi_{m n} \subset \beta_{m n}=\beta\left(\left\{E_{i}^{\frac{1}{m}} \cap E_{j}^{\frac{1}{n}}\right\},\left\{\delta_{m n i j}\right\}, \Delta_{A_{o}, \frac{1}{m}} \cap \Delta_{A_{o}, \frac{1}{n}}\right)
$$

be a division od $[a, b]$ (see Lemma 4.2'). Then $\pi_{m n} \subset \beta_{m}$ and $\pi_{m n} \subset \beta_{n}$. But $\pi_{m} \subset \beta_{m}$ and $\pi_{n} \subset \beta_{n}$, so

$$
\left|s\left(f ; \pi_{m}\right)-s\left(f ; \pi_{m n}\right)\right|<\frac{1}{m} \quad \text { and } \quad\left|s\left(f ; \pi_{n}\right)-s\left(f ; \pi_{m n}\right)\right|<\frac{1}{n}
$$

imply that

$$
\left|s\left(f ; \pi_{m}\right)-s\left(f ; \pi_{n}\right)\right|<\frac{1}{m}+\frac{1}{n}<\epsilon, .
$$


hence $\left\{s\left(f ; \pi_{k}\right)\right\}_{k}$ is a Cauchy sequence. Let $I=\lim _{k \rightarrow \infty} s\left(f ; \pi_{k}\right)$. Fix $m \geq k_{\epsilon}$. Then

$$
\left|s\left(f ; \pi_{m}\right)-I\right|<\frac{1}{m}
$$

Now we show that $I$ satisfies the conditions in Definition 7.1'. Let $\Delta_{B_{o}, \frac{1}{k}}=\Delta_{B_{o}, \frac{1}{k}} / B_{k}$ and let $\alpha \subset \beta_{m}^{* *}=\beta\left(\left\{E_{i}^{\frac{1}{k}}\right\},\left\{\delta_{i}^{\frac{1}{k}}\right\}, \Delta_{A_{k}, \frac{1}{k}} \vee \Delta_{B_{k}, \frac{1}{k}}\right) \subset \beta_{m}$ be a division of $[a, b]$. Then

$$
|s(f ; \alpha)-I| \leq\left|s(f ; \alpha)-s\left(f ; \pi_{m}\right)\right|+\left|s\left(f ; \pi_{m}\right)-I\right|<\frac{1}{m}+\frac{1}{m}<\epsilon .
$$

Lemma 7.3'. Let $\mathcal{S}=\{\mathcal{S}(x)\}) x \in \mathbb{R}$ be a local system $\mathcal{S}_{\infty, \infty}$-filtering. Let $f: \mathbb{R} \rightarrow \mathbb{R}$.

(i) If $a<c<b, f$ is $[\mathcal{S R}]$ integrable on $[a, c]=\left[a_{1}, b_{1}\right]$ to $I_{1}$, and $f$ is $[\mathcal{S R}]$ integrable on $[c, b]=\left[a_{2}, b_{2}\right]$ to $I_{2}$, then $f$ is $[\mathcal{S R}]$ integrable on $[a, b]$ to $I_{1}+I_{2}$.

(ii) If $a \leq c<d \leq b$ and $f$ is $[\mathcal{S R}]$ integrable on $[a, b]$ then $f$ is $] \mathcal{S R}]$ integrable on $[c, d]$.

Proof. (i) Let $\epsilon>0$. For $\epsilon / 2$ let $\left\{E_{i}^{k, \frac{\epsilon}{2}}\right\}$ be a closed $\left[a_{k}, b_{k}\right]$-form, $\delta_{i}^{k, \frac{\epsilon}{2}}: E_{i}^{k, \frac{\epsilon}{2}} \rightarrow(0,+\infty)$ be given by Definition $7.1^{\prime}, k=1,2$. Clearly $\left\{E_{i}^{k, \frac{\epsilon}{2}}\right\}_{i, k}$ is a closed $[a, b]$-form. Let $A \supset$ $\cup_{k=1}^{2} \cup_{i=1}^{\infty} \operatorname{Is}\left(E_{i}^{k, \frac{\epsilon}{2}}\right)$ be a countable set and $B \supset A$ another countable set. Let $A_{k}=$ $A \cap\left[a_{k}, b_{k}\right], B_{k}=B \cap\left[a_{k}, b_{k}\right], k=1,2$. Clearly $B_{k} \supset A_{k} \supset \cup_{i=1}^{\infty} \operatorname{Is}\left(E_{i}^{k, \frac{\epsilon}{2}}\right)$. Again by Definition $7.1^{\prime}$, there exist $\Delta_{A_{k}, \frac{\epsilon}{2}}$ and $\Delta_{B_{k}}, \frac{\epsilon}{2}$ such that

$$
\left|s\left(f ; \pi_{k}^{\prime}\right)-s\left(f ; \pi_{k}^{\prime \prime}\right)\right|<\frac{\epsilon}{2}, \quad k=1,2
$$

whenever $\pi_{k}^{\prime}, \pi_{k}^{\prime \prime} \subset \beta_{k}=\beta\left(\left\{E_{i}^{k, \frac{\epsilon}{2}}\right\},\left\{\delta_{i}^{k, \frac{\epsilon}{2}}\right\}, \Delta_{A_{k}, \frac{\epsilon}{2}} \vee \Delta_{B_{k}, \frac{\epsilon}{2}}\right)$ are divisions of $[a, b]$. Let

$$
\Delta_{A, \epsilon}(x)= \begin{cases}\Delta_{A_{1}, \frac{\epsilon}{2}}(a) \cap(-\infty, c) & \text { if } x=a \\ \Delta_{A_{1}, \frac{\epsilon}{2}}(x) \cap(a, c) & \text { if } x \in A_{1} \cap(a, c) \\ \Delta_{A_{2}, \frac{\epsilon}{2}}(x) \cap(c, b) & \text { if } x \in A_{2} \cap(c, b) \\ \left(\Delta_{A_{1}, \frac{\epsilon}{2}}(c) \cap(a, c]\right) \cup\left(\Delta_{A_{2}, \frac{\epsilon}{2}}(c) \cap[c, b)\right) & \text { if } x=c \\ \Delta_{A_{2}, \frac{\epsilon}{2}}(b) \cap(c,+\infty) & \text { if } x=b .\end{cases}
$$

Let

and

$$
\delta_{i}^{1, \epsilon}(x)= \begin{cases}\min \left\{\delta_{i}^{1, \frac{\epsilon}{2}}(x), c-x\right\} & \text { if } x \in E_{i}^{1, \frac{\epsilon}{2}} \cap[a, c) \\ \delta_{i}^{1, \frac{\epsilon}{2}}(c) & \text { if } x=c \in E_{i}^{1, \frac{\epsilon}{2}}\end{cases}
$$

$$
\delta_{i}^{2, \epsilon}(x)= \begin{cases}\min \left\{\delta_{i}^{2, \frac{\epsilon}{2}}(x), x-c\right\} & \text { if } x \in E_{i}^{2, \frac{\epsilon}{2}} \cap(c, b] \\ \delta_{i}^{2, \frac{\epsilon}{2}}(c) & \text { if } x=c \in E_{i}^{2, \frac{\epsilon}{2}} .\end{cases}
$$

For $B, \Delta_{B, \epsilon}(x)$ is defined similarly with $\Delta_{A, \epsilon}(x)$.

Let $E_{i}^{k, \epsilon}=E_{i}^{k, \frac{\epsilon}{2}}$. Let $\pi \subset \beta=\beta\left(\left\{E_{i}^{k, \epsilon}\right\}_{i, k},\left\{\delta_{i}^{k, \epsilon}\right\}_{i, k}, \Delta_{A, \epsilon} \vee \Delta_{B, \epsilon}\right)$ be a division of $[a, b]$, and let $(\langle x, y\rangle, x) \in \pi$. Then we have: 
1) $x<c \Rightarrow y<c \Rightarrow\langle x, y\rangle \subset[a, c) \Rightarrow(\langle x, y\rangle, x) \in \beta_{1}$;

2) $x>c \Rightarrow y>c \Rightarrow\langle x, y\rangle \subset(c, b] \Rightarrow(\langle x, y\rangle, x) \in \beta_{2}$;

3) $x=c$. Let $c_{1}=\sup _{(\langle x, y\rangle, x) \in \pi}\{x, y\}$ and $c_{2}=\inf _{\substack{(\langle x, y\rangle, x) \in \pi \\ x>c}}\{x, y\}$. Then $\left(\left[c_{1}, c\right], c\right)$ and

$\left(\left[c, c_{2}\right], c\right)$ belong to $\pi$. Note that $\left(\left[c_{1}, c\right], c\right) \in \beta_{1}$ and $\left(\left[c, c_{2}\right], c\right) \in \beta_{2}$.

Let $\pi_{k}=\pi \cap\left[a_{k}, b_{k}\right], k=1,2$. Since $\pi=\pi_{1} \cup \pi_{2}$, by (14) we have

$$
\left|s(f ; \pi)-\left(I_{1}+I_{2}\right)\right| \leq\left|s\left(f ; \pi_{1}\right)-I_{1}\right|+\left|s\left(f ; \pi_{2}\right)-I_{2}\right|<\epsilon .
$$

It follows that $f$ is $[\mathcal{S R}]$ integrable on $[a, b]$ to $I_{1}+I_{2}$.

(ii) Consider $a<c<d<b,[a, c]=\left[a_{1}, b_{1}\right],[c, d]=\left[a_{2}, b_{2}\right],[d, b]=\left[a_{3}, b_{3}\right]$. Suppose that $f$ is $[\mathcal{S R}]$ integrable to $I$ on $[a, b]$. For $\epsilon>0$ there exist a closed $[a, b]$-form $\left\{E_{i}\right\}$, $\delta_{i}: E_{i} \rightarrow(0,+\infty)$, for $A \supseteq \cup_{i=1}^{\infty} \operatorname{Is}\left(E_{i}\right), A$ countable, there is a $\Delta_{A, \epsilon}$, and for $B \supset A, B$ countable, there is a $\Delta_{B, \epsilon}$ such that $|s(f ; \pi)-I|<\epsilon$, whenever $\pi \subset \beta=\beta\left(\left\{E_{i}\right\},\left\{\delta_{i}\right\}, \Delta_{A, \epsilon} \vee\right.$ $\left.\Delta_{B, \epsilon}\right)$ is a division of $[a, b]$. Let $E_{i k}=\left[a_{k}, b_{k}\right] \cap E_{i}, k=1,2,3$. Then $\left\{E_{i k}\right\}$ is a closed $\left[a_{k}, b_{k}\right]$-form, $k=1,2,3$. Let $\delta_{i k}: E_{i k} \rightarrow(0,+\infty), \delta_{i k}(x)=\delta_{i \mid E_{i k}}$. Let $\left[a_{k}, b_{k}\right] \supseteq A_{k} \supset$ $\cup_{i=1}^{\infty} \operatorname{Is}\left(E_{i k}\right), k=1,2,3, A_{k}$ countable, and let $\left[a_{k}, b_{k}\right] \supseteq B_{2} \supset A_{2}$ be another countable set. Let $A=A_{1} \cup A_{2} \cup A_{3}$ and $B=A_{1} \cup B_{2} \cup A_{3}$. Clearly $A \supset \cup_{i=1}^{\infty} \operatorname{Is}\left(E_{i}\right)$. Let $\Delta_{A_{k}, \epsilon}=\Delta_{A, \epsilon} / A_{k}$, $k=1,2,3$ and $\Delta_{B_{2}, \epsilon}=\Delta_{B, \epsilon} / B_{2}$. Let

$$
\pi_{2}^{\prime}, \pi_{2}^{\prime \prime} \subset \beta_{2}=\beta\left(\left\{E_{i 2}\right\},\left\{\delta_{i 2}\right\}, \Delta_{A_{2}, \epsilon} \vee \Delta_{B_{2}, \epsilon}\right) \subset \beta
$$

be a division of $\left[a_{2}, b_{2}\right]$, and let $\pi_{k} \subset \beta_{k}=\beta\left(\left\{E_{i k}\right\},\left\{\delta_{i k}\right\}, \Delta_{A_{k}, \epsilon}\right) \subset \beta$ be a division of $\left[a_{k}, b_{k}\right], k=1,3$. Then $\pi_{1} \cup \pi_{2}^{\prime} \cup \pi_{3}$ and $\pi_{1} \cup \pi_{2}^{\prime \prime} \cup \pi_{3}$ are divisions of $[a, b]$. It follows that

$$
\begin{aligned}
& \left|s\left(f ; \pi_{2}^{\prime}\right)-s\left(f ; \pi_{2}^{\prime \prime}\right)\right|=\left|s\left(f ; \pi_{1} \cup \pi_{2}^{\prime} \cup \pi_{3}\right)-s\left(f ; \pi_{1} \cup \pi_{2}^{\prime \prime} \cup \pi_{3}\right)\right|< \\
& \quad<\left|s\left(f ; \pi_{1} \cup \pi_{2}^{\prime} \cup \pi_{3}\right)-I\right|+\left|s\left(f ; \pi_{1} \cup \pi_{2}^{\prime \prime} \cup \pi_{3}\right)-I\right|<2 \epsilon .
\end{aligned}
$$

By Lemma $7.2^{\prime}, f$ is $[\mathcal{S R}]$ integrable on $\left[a_{2}, b_{2}\right]=[c, d]$.

Lemma 7.4' (A quasi Saks-Henstock Lemma). Let $\mathcal{S}=\{\mathcal{S}(x)\}) x \in \mathbb{R}$ be a local system $\mathcal{S}_{\infty, \infty}$-filtering on $[a, b]$. Let $f: \mathbb{R} \rightarrow \mathbb{R}$ be $[\mathcal{S R}]$ integrable to $I$ on $[a, b]$. Let $F: \mathbb{R} \rightarrow \mathbb{R}$,

$$
F(x)= \begin{cases}0 & \text { if } x \leq a \\ {[\mathcal{S R}] \int_{a}^{x} f(t) d t} & \text { if } x \in(a, b) \\ I & \text { if } x \geq b\end{cases}
$$

Let $\left\{E_{i}\right\}$ be a closed $[a, b]$-form, and let $\delta_{i}: E_{i} \rightarrow(0,+\infty)$ be given for $\epsilon>0$ and $I$. Let $A_{o}$ be a fixed countable subset of $[a, b], A_{o} \supseteq \cup_{i=1}^{\infty} I s\left(E_{i}\right)$, and let $B$ be another countable subset of $[a, b]$ containing $A_{o}$. Let $\Delta_{A_{o}, \epsilon}$ and $\Delta_{B, \epsilon}$ be such that

$$
|s(f ; \pi)-I|<\epsilon,
$$

whenever $\pi \subset \beta=\beta\left(\left\{\left\{E_{i}\right\},\left\{\delta_{i}\right\}, \Delta_{A_{o}, \epsilon} \vee \Delta_{B, \epsilon}\right)\right.$ is a division of $[a, b]$. Then we have:

(i) $|s(f ; \alpha)-S(F ; \pi)|<\frac{3 \epsilon}{2}$ whenever $\alpha \subset \beta^{*}=\beta\left(\left\{E_{i}\right\},\left\{\delta_{i}\right\}, \Delta_{A_{o}, \epsilon}\right)$ is a finite packing;

(ii) $\sum_{(\langle x, y\rangle, x) \in \alpha}|f(x)(y-x)-(F(y)-F(x))|<3 \epsilon$ whenever $\alpha \subset \beta^{*}$ is a finite packing.

Proof. (i) Let $\alpha \subset \beta^{*}$ be a finite packing. If $\alpha$ is a division of $[a, b]$ then we have nothing to prove. Suppose that $[a, b]-\sigma(\alpha) \neq \emptyset$. Let $\left(c_{k}, d_{k}\right), k=1,2, \ldots, n$ be the components of the open set $(a, b) \backslash \sigma(\alpha)$. By Lemma $7.3^{\prime}$ we have

$$
[\mathcal{S R}] \int_{c_{k}}^{d_{k}} f(t) d t=F\left(d_{k}\right)-F\left(c_{k}\right)=I_{k}
$$


For $\frac{\epsilon}{2 n}$ and $I_{k}$, let $\left\{P_{i}^{k}\right\}$ be a closed $\left[c_{k}, d_{k}\right]$-form, $k=1,2, \ldots, n$, and $\eta_{i}^{k}: P_{i}^{k} \rightarrow(0,+\infty)$ be given by Definition $7.1^{\prime}$. Let $E_{i}^{k}=\left[c_{k}, d_{k}\right] \cap E_{i}, k=1,2, \ldots, n, \delta_{i}^{k}: E_{i}^{k} \rightarrow(0,+\infty)$, $\delta_{i}^{k}=\delta_{i \mid E_{i}^{k}}$. Let $B_{k}$ be a countable subset of $\left[c_{k}, d_{k}\right]$ containing $\cup_{i, j} \operatorname{Is}\left(P_{i}^{k} \cap E_{j}^{k}\right)$. Again by Definition $7.1^{\prime}$, for $B_{k}$ and $\frac{\epsilon}{2 n}$ there is a $\Delta_{B_{k}, \frac{\epsilon}{2 n}}$ such that

$$
\left|s\left(f ; \pi_{k}\right)-I_{k}\right|<\frac{\epsilon}{2 n},
$$

whenever $\pi_{k} \subset \beta\left(\left\{P_{i}^{k}\right\},\left\{\eta_{i}^{k}\right\}, \Delta_{B_{k}, \frac{\epsilon}{2 n}}\right)$ is a division of $\left[c_{k}, d_{k}\right]$. Let $B=A_{o} \cup\left(\cup_{k=1}^{n} B_{k}\right)$ and let $\Delta_{B, \epsilon}$ be such that

$$
|s(f ; \pi)-I|<\epsilon,
$$

whenever $\pi \subset \beta=\beta\left(\left\{E_{i}\right\},\left\{\delta_{i}\right\}, \Delta_{A, \epsilon} \vee \Delta_{B, \epsilon}\right)$ is a division of $[a, b]$. Let

$$
\pi_{k}^{*} \subset \beta\left(\left\{P_{i}^{k} \cap E_{j}^{k}\right\},\left\{\delta_{i j}^{k}\right\},\left(\Delta_{B, \epsilon}\right)_{\mid B_{k}} \cap \Delta_{B_{k}, \frac{\epsilon}{2 n}}\right)
$$

be a division of $\left[c_{k}, d_{k}\right]$ (see Lemma 7.2'). Clearly $\alpha \cup\left(\cup_{k=1}^{n} \pi_{k}^{*}\right) \subset \beta$ is a division of $[a, b]$. Hence

$$
\left|s\left(f ; \alpha \cup\left(\cup_{k=1}^{n} \pi_{k}^{*}\right)\right)-I\right|<\epsilon \text { and }\left|s\left(f ; \pi_{k}^{*}\right)-I_{k}\right|<\frac{\epsilon}{2 n} .
$$

Since $I=S(F ; \alpha)+\sum_{k=1}^{n} S\left(F ; \pi_{k}^{*}\right)=S(F ; \alpha)+\sum_{k=1}^{n} I_{k}$, it follows that

$$
\begin{aligned}
& |s(f ; \alpha)-S(F ; \alpha)|=\left|s\left(f ; \alpha \cup\left(\cup_{k=1}^{n} \pi_{k}^{*}\right)\right)-I-\sum_{k=1}^{n}\left(s\left(f ; \pi_{k}^{*}\right)-I_{k}\right)\right| \leq \\
& \leq\left|s\left(f ; \alpha \cup\left(\cup_{k=1}^{n} \pi_{k}^{*}\right)\right)-I\right|+\sum_{k=1}^{n}\left|s\left(f ; \pi_{k}^{*}\right)-I_{k}\right|<\epsilon+n \cdot \frac{\epsilon}{2 n}=\frac{3 \epsilon}{2} .
\end{aligned}
$$

(ii) This follows by definitions using (i).

\section{References}

[1] V. Ene, Real functions - current topics, Lect. Notes in Math., vol. 1603, Springer-Verlag, 1995.

[2] V. Ene, On Borel measurable functions that are $V B G$ and $(N)$, Real Analysis Exchange, 22 (1996/97), no. 2, 688-695.

[3] T. Filipczak, Intersection conditions for some density and I density local systems, Real Analysis Exchange 15 (1989/90), no. 1, 170-192.

[4] R. Gordon, Some comments on an approximately continuous Khintchine integral, Real Analysis Exchange 20 (1994/95), no. 2, 831-841.

[5] R. Henstock, Linear analysis, Butterworth London, 1968.

[6] R. Henstock, A Riemann type integral of Lebesgue power, Can. J. Math. 20 (1968), 79-87. 
[7] R. Henstock, The general theory of integration, Clarendon-Press-Oxford, 1991.

[8] Y. Kubota, An integral of Denjoy type, Proc. Japan Acad. 40 (1964), $713-717$.

[9] Y. Kubota, An elementary theory of the special Denjoy integral, Math. Japon. 24 (1979-1980), 507-520.

[10] Y. Kubota, $A$ direct proof that the $R C$-integral is equivalent to the $D^{*}$ integral, Proc. Amer. Math. Soc. 80 (1980), 293-296.

[11] P. Y. Lee, Lanzhou lectures on Henstock integration, World Scientific, Singapore, 1989.

[12] P. Y. Lee and B. Soedijono, The Kubota integral, Math. Jap. 36 (1991), no. 2, 263-270.

[13] P. Y. Lee and N. I. Wittoya, A direct proof that the Henstock and Denjoy integrals are equivalent, Bull. Malaysian Math. Soc. 5 (1988), no. 2, 43-47.

[14] J. Ridder, Über die gegenseitigen Beziehungen verschiedener stetigen Denjoy-Perron Integrale, Fund. Math. 22 (1934), 136-162.

[15] S. Saks, Theory of the integral, 2nd. rev. ed., vol. PWN, Monografie Matematyczne, Warsaw, 1937.

[16] D. N. Sarkhel, A wide Perron integral, Bull. Austral. Math. Soc. 34 (1986), 233-251.

[17] D. N. Sarkhel, A wide constructive integral, Math. Japonica 32 (1987), 295-309.

[18] D. N. Sarkhel and A. K. De, The proximally continuous integrals, J. Austral. Math. Soc. (Series A) 31 (1981), 26-45.

[19] D. N. Sarkhel and A. B. Kar, (PVB) functions and integration, J. Austral. Math. Soc. (Series A) 36 (1984), 335-353.

[20] B. S. Thomson, Derivation bases on the real line, I and II, Real Analysis Exchange 8 (1982/83), 67-208 and 280-442.

[21] B. S. Thomson, Real functions, Lect. Notes in Math., vol. 1170, SpringerVerlag, 1985.

[22] B. S. Thomson, Derivates of interval functions, Mem. Amer. Math. Soc 93 (1991). 